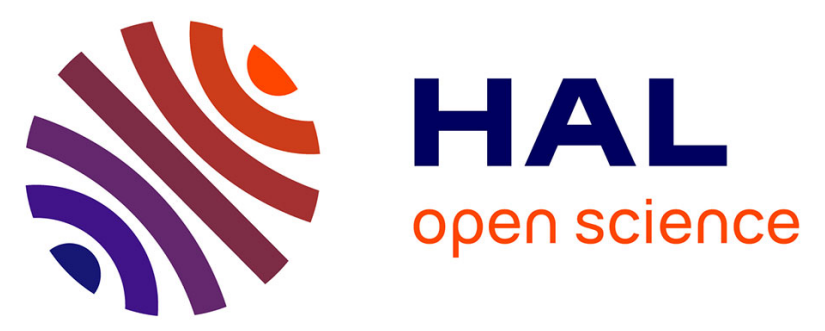

\title{
Certified numerical algorithm for isolating the singularities of the plane projection of generic smooth space curves
}

George Krait, Sylvain Lazard, Guillaume Moroz, Marc Pouget

\section{To cite this version:}

George Krait, Sylvain Lazard, Guillaume Moroz, Marc Pouget. Certified numerical algorithm for isolating the singularities of the plane projection of generic smooth space curves. Journal of Computational and Applied Mathematics, 2021, 394, pp.113553. 10.1016/j.cam.2021.113553 . hal-03161393

\section{HAL Id: hal-03161393 \\ https://hal.inria.fr/hal-03161393}

Submitted on 6 Mar 2021

HAL is a multi-disciplinary open access archive for the deposit and dissemination of scientific research documents, whether they are published or not. The documents may come from teaching and research institutions in France or abroad, or from public or private research centers.
L'archive ouverte pluridisciplinaire $\mathbf{H A L}$, est destinée au dépôt et à la diffusion de documents scientifiques de niveau recherche, publiés ou non, émanant des établissements d'enseignement et de recherche français ou étrangers, des laboratoires publics ou privés. 


\title{
Certified numerical algorithm for isolating the singularities of the plane projection of generic smooth space curves
}

\author{
George Krait ${ }^{1}$, Sylvain Lazard ${ }^{1}$, Guillaume Moroz ${ }^{1}$, and Marc Pouget ${ }^{1}$ \\ Université de Lorraine, CNRS, Inria, LORIA, F-54000 Nancy
}

\begin{abstract}
Isolating the singularities of a plane curve is the first step towards computing its topology. For this, numerical methods are efficient but not certified in general. We are interested in developing certified numerical algorithms for isolating the singularities. In order to do so, we restrict our attention to the special case of plane curves that are projections of smooth curves in higher dimensions. This type of curve appears naturally in robotics applications and scientific visualization. In this setting, we show that the singularities can be encoded by a regular square system whose solutions can be isolated with certified numerical methods. Our analysis is conditioned by assumptions that we prove to be generic using transversality theory. We also provide a semi-algorithm to check their validity. Finally, we present experiments, some of which are not reachable by other methods, and discuss the efficiency of our method.
\end{abstract}

Keywords: Transversality, Generic Singularities, Certified Numerical Algorithms, Interval Analysis, Singular Curve Topology

\section{Introduction}

The problem of computing the topology of a real plane curve consists of computing a piecewise-linear plane graph that can be deformed continuously into that curve. Such a problem is critical for drawing plane curves with the correct topology. One of the main challenges is to isolate the singular points efficiently and correctly. The aim of this paper is to do so with certified numerical methods and we show that this could be achieved for the specific class of plane curves that are projections of $C^{\infty}$ smooth curves in higher dimension.

Although this class of curves seems specific, it appears naturally in visualization and robotic applications and the curves from this class often contain singularities. When visualizing a curve given by $n-1$ implicit equations in $n$ dimensions for instance, we compute its projection in $2 \mathrm{D}$ to display it on a screen. This class of curves also appears in robotic applications. For instance given a robot with two degrees of freedom that moves in the plane, the set of points it can reach is bounded by a curve. In this case, computing the correct topology of this curve is often needed for deciding if a specific position is reachable. This curve is usually the projection of a smooth curve embedded in a space of higher dimension, and it often contains singular points.

Email address: 1 (Firstname.Name@inria.fr) 
By certified algorithms, we refer to algorithms that always output mathematically correct results in a given model of computation; for instance, randomized Las-Vegas algorithms are (usually) certified, but randomized Monte-Carlo algorithms are not; numerical methods that may miss solutions or output spurious solutions are not certified. We consider in this paper the RAM model of computation. Recall that the singular points of a plane curve, defined by the equation $f(x, y)=0$, are the solutions of the system defined by $f(x, y)=\frac{\partial f}{\partial x}(x, y)=$ $\frac{\partial f}{\partial y}(x, y)=0$; it should be stressed that this system is over-determined, i.e., it has more equations than variables, which prevents the use of certified numerical methods such as interval Newton methods [MKC09]. On the other hand, symbolic methods can solve such over-determined systems but they are restricted to algebraic systems and their complexity is high with respect to the degree of the equations.

Main contributions. In this paper, we present a square and regular system that encodes the singularities of the plane projection of a $C^{\infty}$ smooth curve in $\mathbb{R}^{n}$ (Theorems 11 \& 27). Our approach does not use elimination theory to compute the equation of the projected curve and it is not restricted to the algebraic case: it applies to the larger class of $C^{\infty}$ smooth curves. Being square and regular, this system can thus be solved with state-of-the-art certified numerical methods based on interval arithmetic or certified homotopy tracking. However it encodes the singularities of the plane projection only if some assumptions, defined in Section 2.4, are satisfied. Our second main result is that those assumptions are satisfied generically, which we prove using transversality theory (Section 7). We also present Semi-algorithm 4 that checks whether a given curve satisfies our assumptions, that is, an algorithm that stops if and only if the assumptions are satisfied. The combination of these results provides a method that is both numerical and certified for isolating the singularities of the plane projection of a generic curve. Finally, we present several experiments and discuss the efficiency of our algorithm in Section 6 . Our contribution is a generalization of [IMP16b] that only considers the 3-dimensional case and is in the same spirit as the work of Delanoue et al. [DL14].

We also address the case of curves that are the silhouettes of smooth surfaces in $\mathbb{R}^{n}$ (the silhouette being the set of points on the surface where the tangent plane projects on the plane of projection in a line or a point). Such curves naturally appear in parametric systems since they partition the parametric space with respect to the number of solutions of the system. For such curves, we were only able to prove some partial results on their genericity (see Section 2.4. Proposition 61 and Conjecture 62, but our other main results hold (Theorems $11 \& 27$ and our semi-algorithms).

State of the art. The problem of isolating the singularities of a plane curve is a special case of the problem of isolating the solutions of a zero-dimensional system in $\mathbb{R}^{2}$. We give a concise summary of the state of the art of certified methods for these two problems, organized in two main classes.

Symbolic methods. Symbolic methods are widely used for solving in a certified way zero-dimensional algebraic systems. Classical such methods are based on Gröbner bases, resultant theory and univariate representations (see e.g., [CLO92, BPR06]). In this context, methods dedicated to the bivariate case have also been designed (see 
[Hon96, GVK96, BLM $^{+}$16, vdHL18] and references therein). Compared to numerical methods, these methods are adapted to over-constrained or non-regular systems. On the other hand, they suffer several drawbacks. They are not adaptive in the sense that solving in a small region is not easier than solving for all solutions. They are limited to algebraic systems and their complexity is high with respect to the degree of the system.

Certified numerical methods. When a zero-dimensional system is regular (Definition 1), its solutions can be isolated in a certified way using interval-arithmetic subdivision methods [Neu91 MKC09] or homotopy approaches with certified path tracking (see [BL13] and references therein). However, these methods do not directly work for isolating the singularities of a plane curve given by the equation $f(x, y)=0$ because the system $f(x, y)=$ $\frac{\partial f}{\partial x}(x, y)=\frac{\partial f}{\partial y}(x, y)=0$ that encodes the singularities is neither square nor regular. On the other hand, the curve may not be given by its implicit equation and computing this representation may not be required nor desirable. There are only few contributions designing certified numerical algorithms, even with additional restrictions on the curve and its singularities. When the curve is algebraic, Burr et al. [BCGY12] use separation bounds to isolate the singularities via a subdivision algorithm but the worst-case values of such bounds make it inefficient in practice. Lien et al. [LSVY14, LSVY20] study the special case of a singular curve that is a union of non-singular ones such that the singularities are only transverse intersections between them. They propose a subdivision algorithm using the Moore-Kioustelidis interval test for isolating the square and regular system defined by two curves. No implementation is available but such a subdivision scheme in two dimensions is expected to be efficient. In the case where the plane curve is defined as a projection, only two contributions present certified numerical approaches for isolating the singularities: Delanoue and Lagrange [DL14] consider the apparent contours of smooth surfaces in $\mathbb{R}^{4}$ and Imbach et al. [IMP16b] handle the plane projections of smooth curves in $\mathbb{R}^{3}$ using a subdivision scheme locally in four dimensions. Even though subdivision approaches may suffer in practice from the curse of dimensionality, Imbach et al. observe experimentally that, for algebraic curves, their approach is more efficient than computing the implicit equation of the projected plane curves and its singularities using symbolic methods.

The rest of the paper is organized as follows: In Section 2, we introduce notation and the assumptions we consider in our approach. In Section 3 , we introduce the so-called Ball system that characterizes the singularities of the plane projection and we prove, in Section 4, that it is regular at its solutions. In Section 5 we provide a semialgorithm to check the assumptions introduced in Section 2 Experiments are presented in Section 6 Finally, in Section 7 we prove the genericity of our assumptions, with a focus on the case of silhouette curves in Section 7.3

\section{Notation and assumptions}

The main technical notation is summarized in Table 3 at the end of the paper. For a positive integer $n$, a closed (resp. open) $n$-box is the Cartesian product of $n$ closed (resp. open) intervals. Assume that $n \geqslant 3$ and let $B$ be an open $n$-box and $\bar{B}$ be the topological closure of $B$ with respect to the usual topology in $\mathbb{R}^{n}$. Let $C^{\infty}\left(\mathbb{R}^{n}, \mathbb{R}^{n-1}\right)$ denote the set of smooth functions (i.e., differentiable infinitely many times) from $\mathbb{R}^{n}$ to $\mathbb{R}^{n-1}$. Consider the function $P=\left(P_{1}, \ldots, P_{n-1}\right) \in C^{\infty}\left(\mathbb{R}^{n}, \mathbb{R}^{n-1}\right)$. We denote by $\mathfrak{C}($ resp. $\overline{\mathfrak{C}})$ the solution set of the 
system $\left\{P_{1}(x)=\cdots=P_{n-1}(x)=0\right\}$, with $x=\left(x_{1}, \ldots, x_{n}\right) \in B$ (resp. with $x \in \bar{B}$ ). Also, consider the projection $\pi_{\mathfrak{C}}$ (resp. $\pi_{\overline{\mathfrak{C}}}$ ) from $\mathfrak{C}$ (resp. $\overline{\mathfrak{C}}$ ) to the $\left(x_{1}, x_{2}\right)$-plane. Unless otherwise stated, the plane projection of a point $x \in \mathbb{R}^{n}$ is $\left(x_{1}, x_{2}\right)$. Our main goal is to compute the cusp points and nodes of $\pi_{\mathfrak{C}}$. If $\overline{\mathfrak{C}}$ is a smooth curve (see the definition below), define $\mathfrak{L}_{\mathrm{c}}$ (resp. $\overline{\mathfrak{L}_{\mathrm{c}}}$ ) as the set of points $q$ in $\mathfrak{C}$ (resp. $\overline{\mathfrak{C}}$ ) where the tangent line, denoted by $T_{q} \mathfrak{C}$, (resp. $\left.T_{q} \overline{\mathfrak{C}}\right)$ is orthogonal to the $\left(x_{1}, x_{2}\right)$-plane. We also define the set $\mathfrak{L}_{\mathrm{n}}\left(\right.$ resp. $\left.\overline{\mathfrak{L}_{\mathrm{n}}}\right)$ to be the set of points $q$ in $\mathfrak{C}$ (resp. $\overline{\mathfrak{C}}$ ) such that the cardinality of the pre-image of $\pi_{\mathfrak{C}}(q)$ under $\pi_{\mathfrak{C}}$ (resp. $\pi_{\overline{\mathfrak{C}}}$ ) is at least two without counting multiplicities. We will see later that, under some generic assumption, $\mathfrak{L}_{\mathrm{c}}\left(\right.$ resp. $\mathfrak{L}_{\mathrm{n}}$ ) is the set of points in $\mathfrak{C}$ that project to cusps (resp. nodes), which explains the subscript c (resp. n).

\subsection{Regular and singular points}

Let $m \geqslant 1$ be an integer, $V$ be a subset of $\mathbb{R}^{m}$ and $p \in V$. We call $p$ a regular (or smooth) point of $V$ if $V$ is a sub-manifold at $p$, that is, there exist a neighborhood $W$ of $p$ in $\mathbb{R}^{m}$, an integer $k>0$ and $k$ smooth functions $\varphi_{1}, \ldots, \varphi_{k}$ defined over $W$, such that $V \cap W$ is the set of solutions of $\left\{\varphi_{1}(x)=\cdots=\varphi_{k}(x)=0\right\}$ in $W$ and the rank of the matrix $\left(\begin{array}{ccc}\frac{\partial \varphi_{1}}{\partial x_{1}} & \ldots & \frac{\partial \varphi_{1}}{\partial x_{m}} \\ \vdots & \vdots & \vdots \\ \frac{\partial \varphi_{k}}{\partial x_{1}} & \ldots & \frac{\partial \varphi_{k}}{\partial x_{m}}\end{array}\right)$ evaluated at $q$ is $k \mid$ Dem00, Definition 2.2.2]. We call this matrix the Jacobian matrix of the system $\left\{\varphi_{1}(x)=\cdots=\varphi_{k}(x)=0\right\}$ and we denote it by $J_{\left(\varphi_{1}, \ldots, \varphi_{k}\right)}$. If $q$ is not a regular point of $V$, we call it a singular point. If all points in $V$ are regular, then $V$ is called regular or smooth. Otherwise, $V$ is called singular.

For $\varphi=\left(\varphi_{1}, \ldots, \varphi_{k}\right) \in C^{\infty}\left(\mathbb{R}^{n}, \mathbb{R}^{k}\right)$, we denote by $T_{q} \varphi$ its derivative (also known as the tangent map) at the point $q$. Note that the Jacobian matrix $J_{\varphi}=J_{\left(\varphi_{1}, \ldots, \varphi_{k}\right)}$ is the expression of the derivative in the canonical bases of $\mathbb{R}^{n}$ and $\mathbb{R}^{k}$

Definition 1. let $F=\left(f_{1}, \ldots, f_{n}\right)$ be a vector of smooth real-valued functions that are defined in $\mathbb{R}^{n}$ and let $a \in \mathbb{R}^{n}$ be a solution of the system $\{F=0\}$. We say that the latter system is regular at $a \in \mathbb{R}^{n}$ if the determinant of its Jacobian matrix, evaluated at a, does not vanish. We call $\{F=0\}$ regular if it is regular at all of its solutions.

\subsection{Multiplicity in zero-dimensional systems}

We recall the definition of multiplicity in the univariate case before generalizing it to higher dimensions.

Definition 2. Let $f$ be a real smooth function at $a \in \mathbb{R}$. The multiplicity of $f$ at $a$ is the integer mult $_{a}(f(x))=$ $\min \left\{k \in \mathbb{N} \mid \frac{\partial^{k} f}{\partial x^{k}}(a) \neq 0\right\}$ if it exists, otherwise $\operatorname{mult}_{a}(f(x))=\infty$. For the case $a=0$, we write $\operatorname{mult}(f)=$ $\operatorname{mult}_{a}(f)$ for simplicity.

Definition 3 ([CLO05, Definition 4.2.1]). For integers $m \geqslant n \geqslant 1$, let $G=\left(g_{1}(x), \ldots, g_{m}(x)\right)$ be a polynomial function from $\mathbb{R}^{n}$ to $\mathbb{R}^{m}$ and $q$ be a solution of the system $\{G=0\}$. Let $\mathbb{R}[x]$ be the ring of polynomials with $n$ variables and define $\mathbb{R}[x]_{q}=\left\{\frac{h_{1}}{h_{2}} \mid h_{1}, h_{2} \in \mathbb{R}[x], h_{2}(q) \neq 0\right\}$ to be the localization of $\mathbb{R}[x]$ at $q$. Define the 
intersection multiplicity of $q$ in the system $\{G=0\}$ (or equivalently the multiplicity of the system $\{G=0\}$ at $q$ ) to be the dimension of the real vector space $\frac{\mathbb{R}[x]_{q}}{I_{G}}$, where $I_{G}$ is the ideal generated by the set $\left\{\frac{g_{1}}{1}, \ldots, \frac{g_{m}}{1}\right\}$ in $\mathbb{R}[x]_{q}$.

The previous definition is classical for the algebraic case. However, in our paper, we are interested in curves defined as the zero locus of smooth functions. For this goal, we consider a more general definition for a system $S=\left\{f_{1}(x)=\cdots=f_{m}(x)=0\right\}$ with $f_{i} \in C^{\infty}\left(\mathbb{R}^{n}, \mathbb{R}\right)$. Let $a$ be a solution of $S$ and $k$ be a non-negative integer, we define the dual space of rank $k$, denoted by $D_{a}^{k}[S]$, to be the vector space of all linear combinations $c$ of differential functionals $\frac{\partial^{k_{1}+\cdots+k_{n}}}{\partial x_{1}^{k_{1}} \ldots \partial x_{n}^{k_{n}}}$ with $k_{1}+\cdots+k_{n} \leqslant k$ such that:

(a) $D_{a}^{0}[S]=\operatorname{span}\left(\left\{\frac{\partial^{0}}{\partial x_{1}^{0} \ldots \partial x_{n}^{0}}\right\}\right)$,

(b) $c$ in $D_{a}^{k}[S]$ applied to $f_{i}$, evaluated at $a$ is zero for all integers $1 \leqslant i \leqslant m$, and

(c) for all $i \in\{1, \ldots, n\}$, the anti-differentiation transformation $\phi_{j}$ applied to $c$ in $D_{a}^{k}[S]$ is in $D_{a}^{k-1}[S]$. The anti-differentiation transformation $\phi_{j}$ is the linear operator mapping the order $h$ differential functional $\frac{\partial^{h}}{\partial x_{1}^{h_{1}} \ldots \partial x_{j}^{h_{j}} \ldots \partial x_{n}^{h_{n}}}$ to the order $(h-1)$ differential functional $\frac{\partial^{h-1}}{\partial x_{1}^{h_{1}} \ldots \partial x_{j}^{h_{j}-1} \ldots \partial x_{n}^{h_{n}}}$ if $h_{j}>0$ or to the order 0 differential functional $\frac{\partial^{0}}{x_{j}^{0}}$ otherwise, where $h=\sum_{i=1}^{n} h_{i}$.

Definition 4 ([DLZ11, Definition 1]). Let $F \in C^{\infty}\left(\mathbb{R}^{n}, \mathbb{R}^{k}\right)$ such that $F^{-1}(0)$ is a finite set and let $a \in \mathbb{R}^{n}$ be a solution of the system $S=\{F=0\}$. Consider the ascending chain of dual spaces $D_{a}^{0}[F] \subseteq D_{a}^{1}[F] \subseteq$ $\ldots D_{a}^{h}[F] \subseteq \ldots$ If there exists an integer $\alpha$ such that $D_{a}^{\alpha}[F]=D_{a}^{\alpha+1}[F]$, then the dimension of the vector space $D_{a}^{\alpha}[F]$ is called the multiplicityof a in the system $S$. If such an $\alpha$ does not exist, the multiplicity is, by convention, infinite.

For polynomial systems, the two definitions are equivalent [DLZ11, Theorem 2] and in addition the following proposition shows that algebraic tools can be used in the smooth case.

Proposition 5 ([DLZ11, Corollary 3]). For an integer $k \geqslant n$, let $F=\left(f_{1}, \ldots, f_{k}\right) \in C^{\infty}\left(\mathbb{R}^{n}, \mathbb{R}^{k}\right)$ and let $a \in \mathbb{R}^{n}$ be a solution of the system $\{F=0\}$. Suppose that the multiplicity of $a$ in $\{F=0\}$ is $m<\infty$, then the intersection multiplicity at a of the polynomial system $\left\{G=\left(g_{1}, \ldots, g_{k}\right)=0\right\}$ is also $m$, where $g_{i}$ is equal to the Taylor expansion of $f_{i}$ at a up to degree at least $m$.

\subsection{Singularities of plane curves, nodes and ordinary cusps}

Definition 6 ([AGZV12, §17.1]). For $i \in\{1,2\}$, let $C_{i}$ be a plane curve defined in a neighborhood $U_{i} \subset \mathbb{R}^{2}$ of $p_{i}$ by the 0 -set of a smooth function $f_{i}$. The pairs $\left(p_{1}, C_{1}\right)$ and $\left(p_{2}, C_{2}\right)$ are equivalent, and thus define the same plane curve singularity, if there exists a diffeomorphism $\varphi$ from $U_{1}$ to $U_{2}$ such that $f_{1}=f_{2} \circ \varphi$ and $\varphi\left(p_{1}\right)=p_{2}$.

In particular, a singularity is of type $A_{k}$ if the curve is locally defined at the origin by the 0-set of the function $x^{2}-y^{k+1}$. As important special cases, $A_{1}$ is called a node singularity and $A_{2}$ is called an ordinary cusp singularity, see Figure 1 

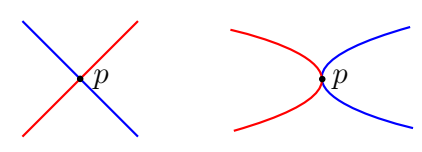

Figure 1: Left: At an $A_{1}$ singularity, two branches of the curve intersect transversally. Right: At an $A_{2 k+1}$ singularity with $k>1$, the tangent lines of the two branches at the intersection point coincide.

\footnotetext{
${ }^{1}$ Note that the converse is not true as the vertical (double) line defined by $x_{1}^{2}=x_{2}=0$ in $\mathbb{R}^{3}$ is smooth but the rank of its Jacobian is never full.
} 


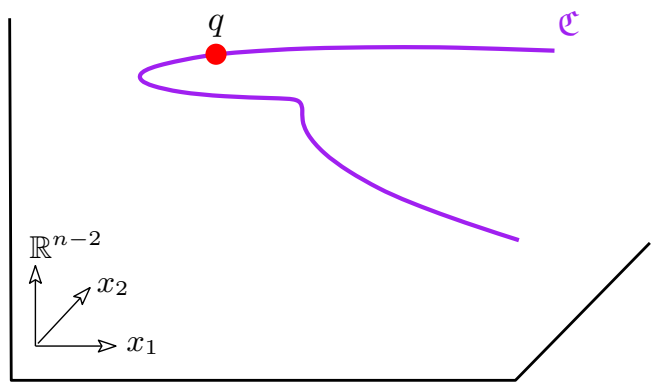

(a) Assumption $\mathcal{A}_{1}$ : $\overline{\mathfrak{C}}$ is a smooth; the rank of the Jacobian of $P$ at $q$ is full.

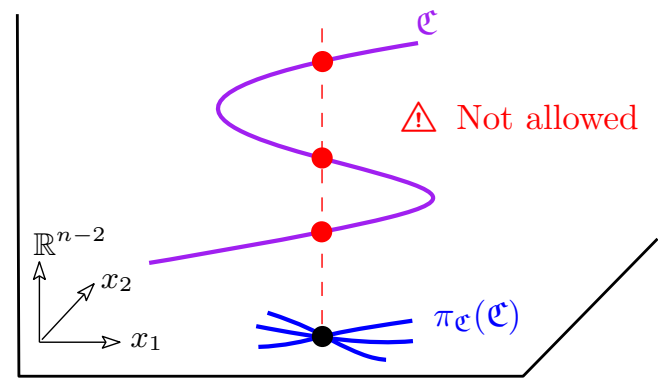

(c) Assumption $\mathcal{A}_{3}$ : No three points (counted with multiplicity) have the same projection.

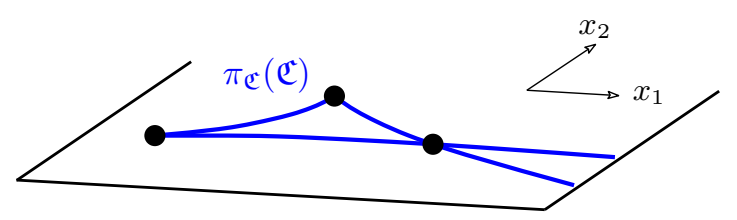

(e) Assumption $\mathcal{A}_{5}^{-}$: points in $\pi_{\mathfrak{C}}(\mathfrak{C})$ are either smooth, nodes or ordinary cusps.

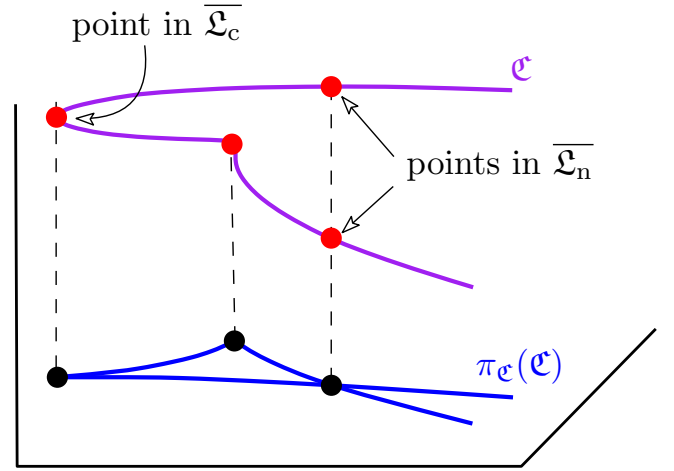

(b) Assumptions $\mathcal{A}_{2}$ and $\mathcal{A}_{4}$ : the sets $\overline{\mathfrak{L}_{\mathrm{c}}}$ and $\overline{\mathfrak{L}_{n}}$ are finite and do not intersect the boundary of $B$.

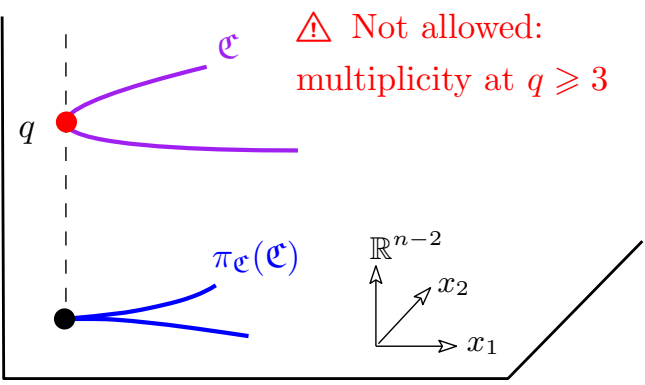

(d) Assumption $\mathcal{A}_{3}$ : No three points (counted with multiplicity) have the same projection.

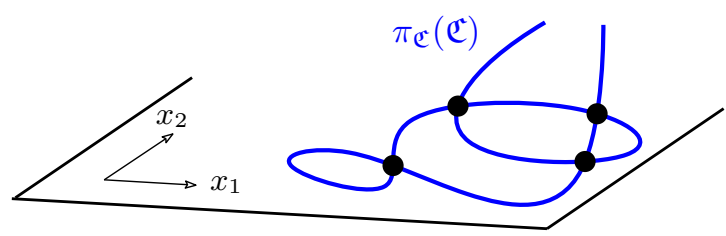

(f) Assumption $\mathcal{A}_{5}$ : points in $\pi_{\mathfrak{C}}(\mathfrak{C})$ are only smooth or nodes.

Figure 2: Illustration of the assumptions. 


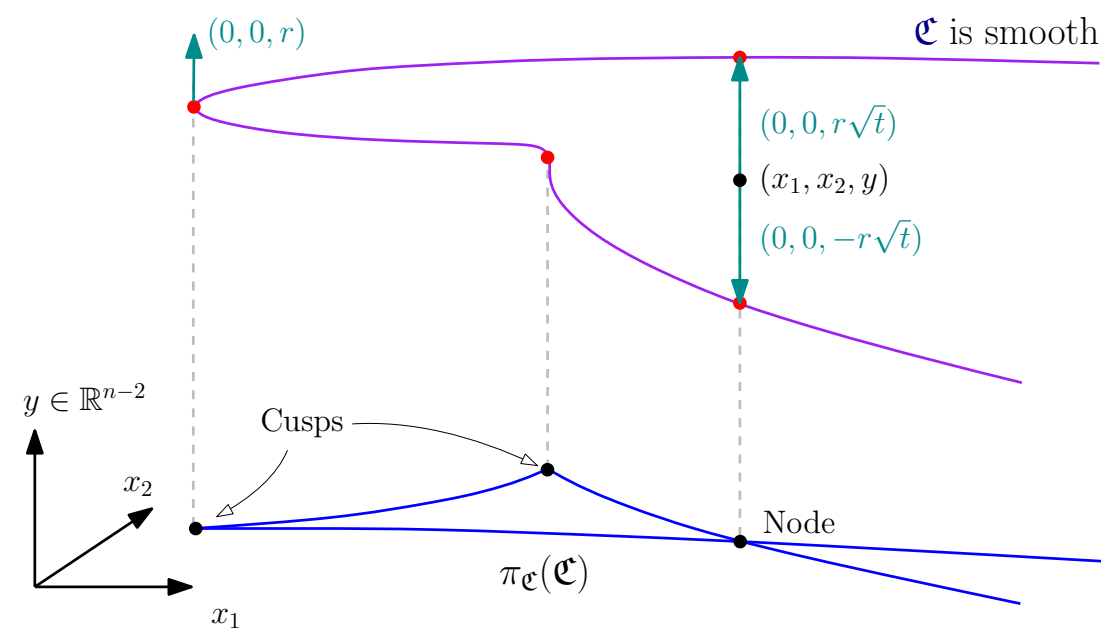

Figure 3: Illustration of a node and cusps in the plane projection of a smooth curve.

Our motivation for also considering these weak assumptions is dual. First, our certified algorithm for isolating the singularities of the projection of curves satisfying the strong assumptions also works, to some extent, if only the weak assumptions hold: namely, it outputs a superset of the isolating boxes of the singularities. Second, we conjecture that our weak assumptions are satisfied by silhouette curves of generic surfaces (see Proposition 61 and Conjecture 62).

\section{Modelling system}

Our goal in this section is, under the weak assumptions, to encode the singularities of the projected curve $\pi_{\mathfrak{C}}(\mathfrak{C})$ by a square and regular (see Definition 1 ) system so that it is solvable with certified numerical methods. In Section 3.1. we first define this system $\operatorname{Ball}(P)$ and state the first main result of this section, Theorem 11, showing that the Ball system exactly encodes the singularities of $\pi_{\mathfrak{C}}(\mathfrak{C})$. In Sections 3.2 and 3.3 , we study the singularities obtained as the projections of the points in $\mathfrak{L}_{n}$ and $\mathfrak{L}_{c}$, respectively. Theorem 11 is proved in Section 3.4 . In Section 4 , we will prove our second main result, Theorem 27, stating necessary and sufficient conditions for the Ball system to be regular.

\subsection{Encoding the singular points of the plane projection}

By Assumption $\mathcal{A}_{5}^{-}$, the singularities of the projected curve $\pi_{\mathfrak{C}}(\mathfrak{C})$ are only nodes and cusps. Intuitively, a node appears when two points of $\mathfrak{C}$ project to the same point and a cusp appears when projecting a point with a tangent line orthogonal to the projection plane (see Figure 3). The idea to encode the nodes is to design a system whose variables are the coordinates of two different points in $\mathbb{R}^{n}$ constrained to be on $\mathfrak{C}$ and so that they have the 
same plane projection. To encode a cusp, we design a system whose variables are the coordinates of one point in $\mathbb{R}^{n}$ constrained to be on $\mathfrak{C}$ with a tangent orthogonal to the projection plane. Furthermore, in order to apply certified numerical methods we need systems that are square and regular (Definition 11). To solve this issue and to gather the two systems into a single one, we first parameterize two different points of $\mathfrak{C}$ with the same projection by $\left(x_{1}, x_{2}, y+r \sqrt{t}\right)$ and $\left(x_{1}, x_{2}, y-r \sqrt{t}\right)$, with $x_{1}, x_{2}, t \in \mathbb{R}, y, r$ in $\mathbb{R}^{n-2}$ and $\|r\|=1$. Then, given any function $f$ from $\mathbb{R}^{n}$ to $\mathbb{R}$ so that $f=0$ is one of the $n-1$ hypersurfaces that define $\mathfrak{C}$, we introduce in Definition 9 two smooth functions $S \cdot f$ and $D \cdot f$. When $t>0$, they return, roughly speaking, the arithmetic mean and difference of $f$ at the above two points, hence they both vanish if and only if the two points are on the hypersurface $f=0$. When $t=0$, the two points coincide and $S \cdot f$ and $D \cdot f$ return, roughly speaking, $f$ evaluated at this point and the gradient of $f$ (at that point) scalar the "vertical" vector $(0,0, r)$; hence, they both vanish if and only if the point is on the hypersurface $f=0$ and its tangent hyperplane is normal to the plane of projection. It follows that given a curve defined by $P_{1}=\cdots=P_{n-1}=0$, the solutions of the so-called Ball system of all $S \cdot P_{i}=D \cdot P_{i}=0$ is the set of points on the curve that project to nodes and cusps (Theorem 11). Note that we consider $\sqrt{t}$ instead of $t$ in the parameterization $\left(x_{1}, x_{2}, y \pm r \sqrt{t}\right)$ for ensuring the regularity of the Ball system when $t=0$ (because this ensures that the linear term of the Taylor expansion of $D \cdot f$ does not vanish).

Definition 9. Let $x_{1}, x_{2}$, t be variables in $\mathbb{R}$ and $y, r$ in $\mathbb{R}^{n-2}$. For a smooth function $f: \bar{B} \subset \mathbb{R}^{n} \rightarrow \mathbb{R}$, we define the functions $S \cdot f$ and $D \cdot f$ from $\mathbb{R}^{2 n-1}$ to $\mathbb{R}$.

$$
S \cdot f\left(x_{1}, x_{2}, y, r, t\right)= \begin{cases}\frac{1}{2}\left[f\left(x_{1}, x_{2}, y+r \sqrt{t}\right)+f\left(x_{1}, x_{2}, y-r \sqrt{t}\right)\right], & \text { for } t>0 \\ f\left(x_{1}, x_{2}, y\right), & \text { for } t=0\end{cases}
$$

and

$$
D \cdot f\left(x_{1}, x_{2}, y, r, t\right)= \begin{cases}\frac{1}{2 \sqrt{t}}\left[f\left(x_{1}, x_{2}, y+r \sqrt{t}\right)-f\left(x_{1}, x_{2}, y-r \sqrt{t}\right)\right], & \text { for } t>0 \\ \nabla f\left(x_{1}, x_{2}, y\right) \cdot(0,0, r), & \text { for } t=0 .\end{cases}
$$

Lemma 10. If $f$ is a smooth function defined on $\bar{B} \subseteq \mathbb{R}^{n}$, then both $S \cdot f$ and $D \cdot f$ are smooth functions on the subset

$$
\bar{B}_{\text {Ball }}=\left\{\left(x_{1}, x_{2}, y, r, t\right) \in \mathbb{R} \times \mathbb{R} \times \mathbb{R}^{n-2} \times \mathbb{R}^{n-2} \times \mathbb{R} \mid t \geqslant 0,\left(x_{1}, x_{2}, y \pm r \sqrt{t}\right) \in \bar{B},\|r\|^{2}=1\right\}
$$

of $\mathbb{R}^{2 n-1}$, where $\|r\|$ denotes the Euclidean norm of $r$.

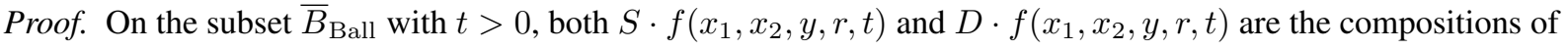
smooth functions, hence they are smooth functions.

For a point $X=\left(x_{1}, x_{2}, y, r, t\right)$ in $B_{\text {Ball }}$ with $t=0$, we will prove that $S \cdot f$ (resp. $\left.D \cdot f\right)$ is a $C^{s}$ function for an arbitrarily $s$ which implies that $S \cdot f$ (resp. $D \cdot f$ ) is smooth. First define the function

$$
S_{0} \cdot f\left(x_{1}, x_{2}, y, r, t\right)= \begin{cases}\frac{1}{2}\left[f\left(x_{1}, x_{2}, y+r t\right)+f\left(x_{1}, x_{2}, y-r t\right)\right], & \text { for } t>0 \\ f\left(x_{1}, x_{2}, y\right), & \text { for } t=0 .\end{cases}
$$

Since $S_{0} \cdot f\left(x_{1}, x_{2}, y, r, t\right)$ is an even smooth function with respect to $t$, the partial derivatives of $S_{0} \cdot f$ with respect to $t$ of odd orders, evaluated at $X$, are zero. For an integer $s>0$, by the parameterized Taylor formula without remainder [Dem00, Proposition 4.2.2], there exist smooth functions $a_{i}\left(x_{1}, x_{2}, y, r\right)$, with integers $0 \leqslant i<s$ such 
that $S_{0} \cdot f\left(x_{1}, x_{2}, y, r, t\right)=\sum_{i=0}^{s-1} a_{i}\left(x_{1}, x_{2}, y, r\right) t^{2 i}+t^{2 s} \cdot \phi\left(x_{1}, x_{2}, y, t\right)$, where $\phi\left(x_{1}, x_{2}, y, t\right)$ is a smooth function.

Notice that $S \cdot f\left(x_{1}, x_{2}, y, r, t\right)=\sum_{i=0}^{s-1} a_{i}\left(x_{1}, x_{2}, y, r\right) t^{i}+t^{s} \cdot \phi\left(x_{1}, x_{2}, y, \sqrt{t}\right)$, so that a partial derivative exists up to order $s$ at $t=0$. Thus, $S \cdot f\left(x_{1}, x_{2}, y, r, t\right)$ is a $C^{s-1}$ function. This holds for any arbitrarily large $s$, hence $S \cdot f\left(x_{1}, x_{2}, y, r, t\right)$ is a $C^{\infty}$ function.

Now, we prove that $D \cdot f$ is continuous at $X=\left(x_{1}, x_{2}, y, r, 0\right)$. Let $X_{i}$ be a sequence that converges to $X$. To prove that $D \cdot f\left(X_{i}\right)$ converges to $D \cdot f(X)$, it is enough to show that for a sequence $t_{i}$ that converges to 0 , then we have that $D \cdot f\left(x_{1}, x_{2}, y, r, t_{n}\right)$ converges to $D \cdot f(X)$. We can assume that $t_{i} \neq 0$ for all $i$, so that

$$
\begin{aligned}
\lim _{t_{i} \rightarrow 0} D \cdot f\left(x_{1}, x_{2}, y, r, t_{i}\right)= & \lim _{t_{i} \rightarrow 0} \frac{1}{2 \sqrt{t_{i}}}\left[f\left(x_{1}, x_{2}, y+r \sqrt{t_{i}}\right)-f\left(x_{1}, x_{2}, y-r \sqrt{t_{i}}\right)\right] \\
= & \lim _{t_{i} \rightarrow 0} \frac{1}{2 \sqrt{t_{i}}}\left[f\left(x_{1}, x_{2}, y+r \sqrt{t_{i}}\right)-\left(f\left(x_{1}, x_{2}, y\right)-f\left(x_{1}, x_{2}, y\right)\right)-f\left(x_{1}, x_{2}, y-r \sqrt{t_{i}}\right)\right] \\
= & \lim _{t_{i} \rightarrow 0} \frac{1}{2 \sqrt{t_{i}}}\left[f\left(x_{1}, x_{2}, y+r \sqrt{t_{i}}\right)-f\left(x_{1}, x_{2}, y\right)\right] \\
& \quad \quad \quad \lim _{t_{i} \rightarrow 0} \frac{1}{2 \sqrt{t_{i}}}\left[f\left(x_{1}, x_{2}, y\right)-f\left(x_{1}, x_{2}, y-r \sqrt{t_{i}}\right)\right] \\
= & \frac{1}{2} \nabla f \cdot(0,0, r)-\frac{1}{2} \nabla f \cdot(0,0,-r) \\
= & \nabla f \cdot(0,0, r) .
\end{aligned}
$$

We now prove that $D \cdot f$ is smooth at $X$. Similarly to the proof of the case of $S \cdot f$, since the function $\frac{1}{2}\left[f\left(x_{1}, x_{2}, y+\right.\right.$ $\left.r t)-f\left(x_{1}, x_{2}, y-r t\right)\right]$ is odd with respect to $t$, there exist smooth functions $b_{i}\left(x_{1}, x_{2}, y, r\right)$, for $1 \leqslant i<s$ and $\psi\left(x_{1}, x_{2}, y, r, t\right)$ such that $\frac{1}{2}\left[f\left(x_{1}, x_{2}, y+r t\right)-f\left(x_{1}, x_{2}, y-r t\right)\right]=\sum_{i=0}^{s-1} b_{i}\left(x_{1}, x_{2}, y, r\right) t^{2 i+1}+t^{2 s+1} \cdot \psi\left(x_{1}, x_{2}, y, t\right)$. Notice that $D \cdot f\left(x_{1}, x_{2}, y, r, t\right)=\sum_{i=0}^{s-1} b_{i}\left(x_{1}, x_{2}, y, r\right) t^{i}+t^{s} \cdot \psi\left(x_{1}, x_{2}, y, \sqrt{t}\right)$, so that a partial derivative exists up to order $s$ at $t=0$. Thus, $D \cdot f\left(x_{1}, x_{2}, y, r, t\right)$ is a $C^{s-1}$ function. This holds for any arbitrarily large $s$, hence $D \cdot f\left(x_{1}, x_{2}, y, r, t\right)$ is a $C^{\infty}$ function.

Theorem 11. Consider $P=\left(P_{1}, \ldots, P_{n-1}\right) \in C^{\infty}\left(\mathbb{R}^{n}, \mathbb{R}^{n-1}\right)$ that satisfies Assumptions $\mathcal{A}_{1}, \mathcal{A}_{2}, \mathcal{A}_{3}$ and $\mathcal{A}_{4}$ Then, $X=\left(x_{1}, x_{2}, y, r, t\right) \in \mathbb{R} \times \mathbb{R} \times \mathbb{R}^{n-2} \times \mathbb{R}^{n-2} \times \mathbb{R}$ is a solution of the Ball system

$$
\operatorname{Ball}(P)=\left\{\begin{array}{l}
S \cdot P_{1}(X)=\cdots=S \cdot P_{n-1}(X)=0 \\
D \cdot P_{1}(X)=\cdots=D \cdot P_{n-1}(X)=0 \\
\|r\|^{2}-1=0
\end{array}\right.
$$

if and only if $\left(x_{1}, x_{2}\right)$ is a singular point of $\pi_{\mathfrak{C}}(\mathfrak{C})$ (see Definition 9 for the notation $S \cdot P_{i}$ and $\left.D \cdot P_{i}\right)$.

We postpone the proof of Theorem 11 to the end of Section 3.3 As a first step, we study a mapping from the solutions of the Ball system to pairs of points on the curve $\mathfrak{C}$. 
Definition 12. Let $P \in C^{\infty}\left(\mathbb{R}^{n}, \mathbb{R}^{n-1}\right)$. Define $\widehat{\mathfrak{L}}_{\mathrm{n}}$ to be the set of pairs $\left(q_{1}, q_{2}\right)$ with $q_{1}, q_{2} \in \mathfrak{C}, q_{1} \neq q_{2}$ and $\pi_{\mathfrak{C}}\left(q_{1}\right)=\pi_{\mathfrak{C}}\left(q_{2}\right)$, also define $\widehat{\mathfrak{L}}_{\mathrm{c}}$ to be the set of pairs $\left(q_{1}, q_{1}\right)$ with $q_{1} \in \mathfrak{L}_{\mathrm{c}}$, and let $\widehat{\mathfrak{L}}=\widehat{\mathfrak{L}}_{\mathrm{n}} \cup \widehat{\mathfrak{L}}_{\mathrm{c}}$.

Lemma 13. Consider $P=\left(P_{1}, \ldots, P_{n-1}\right) \in C^{\infty}\left(\mathbb{R}^{n}, \mathbb{R}^{n-1}\right)$ and let $X=\left(x_{1}, x_{2}, y, r, t\right) \in \mathbb{R} \times \mathbb{R} \times \mathbb{R}^{n-2} \times$ $\mathbb{R}^{n-2} \times \mathbb{R}$, with $\|r\|=1$. Assume that $P$ satisfies Assumption $\mathcal{A}_{1}$. Then $X$ is a solution of $\operatorname{Ball}(P)$ if and only if for the points $q_{1}=\left(x_{1}, x_{2}, y+r \sqrt{t}\right)$ and $q_{2}=\left(x_{1}, x_{2}, y-r \sqrt{t}\right)$, the pair $\left(q_{1}, q_{2}\right)$ is in $\widehat{\mathfrak{L}}_{\mathrm{n}}$, or in $\widehat{\mathfrak{L}}_{\mathrm{c}}$ with $(0,0, r) \in \mathbb{R} \times \mathbb{R} \times \mathbb{R}^{n-2}$ in $T_{q_{1}} \mathfrak{C}$.

Proof. Note that, by Assumption $\mathcal{A}_{1}$ the tangent space to the curve at any of its points is well defined and is a line. First, assume that $X$ is a solution of $\operatorname{Ball}(P)$. We consider two cases:

(a) If $t>0$, then since $r \neq 0 \in \mathbb{R}^{n-2}$ we have that $q_{1} \neq q_{2}$. Moreover, since $S \cdot P_{i}(X)=D \cdot P_{i}(X)=0$ for all $i \in\{1, \ldots, n-1\}$, we deduce that $P_{i}\left(q_{1}\right)=P_{i}\left(q_{2}\right)=0$, thus $q_{1}, q_{2} \in \mathfrak{C}$. Moreover, since $\pi_{\mathfrak{C}}\left(q_{1}\right)=\pi_{\mathfrak{C}}\left(q_{2}\right)=\left(x_{1}, x_{2}\right)$ we have $q_{1}, q_{2} \in \mathfrak{L}_{\mathrm{n}}$. Thus, $\left(q_{1}, q_{2}\right) \in \widehat{\mathfrak{L}}_{\mathrm{n}}$.

(b) If $t=0$, then $q_{1}=q_{2}$. First, $P_{i}\left(q_{1}\right)=S \cdot P_{i}(X)=0$, for all indices $i \in\{1, \ldots, n-1\}$, hence $q_{1} \in \mathfrak{C}$. Moreover, we have that $0=D \cdot P_{i}(X)=\nabla P_{i}\left(q_{1}\right) \cdot(0,0, r)$, for all $i \in\{1, \ldots, n-1\}$, equivalently, $J_{P}\left(q_{1}\right) \cdot(0,0, r)^{T}=0 \in \mathbb{R}^{n-1}$, i.e., we have $(0,0, r) \in T_{q_{1}} \mathfrak{C}$. Thus, $q_{1} \in \mathfrak{L}_{\mathrm{c}}$ and hence, $\left(q_{1}, q_{1}\right) \in \widehat{\mathfrak{L}}_{\mathrm{c}}$.

Now, let us prove the other direction:

(a) If $\left(q_{1}, q_{2}\right) \in \widehat{\mathfrak{L}}_{\mathrm{n}}$, then $q_{1} \neq q_{2}$ and $t \neq 0$. Also, since $q_{1}, q_{2} \in \mathfrak{C}$, we can write that $S \cdot P_{i}(X)=$ $\frac{1}{2}\left(P_{i}\left(q_{1}\right)+P_{i}\left(q_{2}\right)\right)=0$, and $D \cdot P_{i}(X)=\frac{1}{2 \sqrt{t}}\left(P_{i}\left(q_{1}\right)-P_{i}\left(q_{2}\right)\right)=0$, for all $i \in\{1, \ldots, n-1\}$. Thus, $X$ is a solution of $\operatorname{Ball}(P)$.

(b) If $\left(q_{1}, q_{2}\right) \in \widehat{\mathfrak{L}}_{\mathrm{c}}$ and $(0,0, r) \in \mathbb{R} \times \mathbb{R} \times \mathbb{R}^{n-2}$ is in $T_{q_{1}} \mathfrak{C}$, one has $q_{1}=q_{2} \in \mathfrak{L}_{\mathrm{c}} \subseteq \mathfrak{C}$, and $t=0$. Moreover, for all $i \in\{1, \ldots, n-1\}$ we have $S \cdot P_{i}(X)=P_{i}\left(q_{1}\right)=0$ and since $(0,0, r) \in T_{q_{1}} \mathfrak{C}$, we can equivalently write $D \cdot P_{i}(X)=\nabla P_{i}\left(q_{1}\right) \cdot(0,0, r)=0$. Thus, $X$ is a solution of $\operatorname{Ball}(P)$.

Definition 14. Let $\operatorname{Sol}_{B a l l(P)}$ be the solution set of $\operatorname{Ball}(P)$. Define the function $\Omega_{P}$ from $\operatorname{Sol}_{\operatorname{Ball}(P)}$ to $\widehat{\mathfrak{L}}$ that sends $X=\left(x_{1}, x_{2}, y, r, t\right) \in \mathbb{R} \times \mathbb{R} \times \mathbb{R}^{n-2} \times \mathbb{R}^{n-2} \times \mathbb{R}^{+}$to the ordered pair $q_{1}=\left(x_{1}, x_{2}, y+r \sqrt{t}\right)$ and $q_{2}=\left(x_{1}, x_{2}, y-r \sqrt{t}\right)$. Notice that the function $\Omega_{P}$ is well-defined by Lemma 13

Lemma 15. If $P \in C^{\infty}\left(\mathbb{R}^{n}, \mathbb{R}^{n-1}\right)$ satisfies Assumption $\mathcal{A}_{1}$ then $\Omega_{P}$ is surjective.

Proof. For any pair $\left(q_{1}, q_{2}\right) \in \widehat{\mathfrak{L}}_{\mathrm{n}}$ we have that the point $X=\left(\frac{1}{2}\left(q_{1}+q_{2}\right), \frac{\Pi_{\mathfrak{C}}\left(q_{1}-q_{2}\right)}{\left\|q_{1}-q_{2}\right\|}, \frac{1}{4}\left\|q_{1}-q_{2}\right\|^{2}\right) \in \mathbb{R}^{n} \times$ $\mathbb{R}^{n-1} \times \mathbb{R}^{+}$is a solution of $\operatorname{Ball}(P)$, where $\Pi_{\mathfrak{C}}\left(q_{1}-q_{2}\right)$ is the vector in $\mathbb{R}^{n-2}$ obtained by omitting the first two coordinates (which are zeros) from $q_{1}-q_{2}$. Note that $\Omega_{P}(X)=\left(q_{1}, q_{2}\right)$. If the pair $\left(q_{1}, q_{1}\right)$ is in $\widehat{\mathfrak{L}}_{\mathrm{c}}$, we define $r$ in the following way: we take a unit vector $v \in T_{q_{1}} \mathfrak{C}$ (the first two coordinates of $v$ are zeros since $q_{1} \in \mathfrak{L}_{\mathrm{c}}$ ). We set $r$ to be $\Pi_{\mathfrak{C}}(v)$. Again $X=\left(q_{1}, r, 0\right) \in \mathbb{R}^{n} \times \mathbb{R}^{n-2} \times \mathbb{R}$ is a solution of $\operatorname{Ball}(P)$, with $\Omega_{P}(X)=\left(q_{1}, q_{1}\right)$. Thus, $\Omega_{P}$ is surjective. 


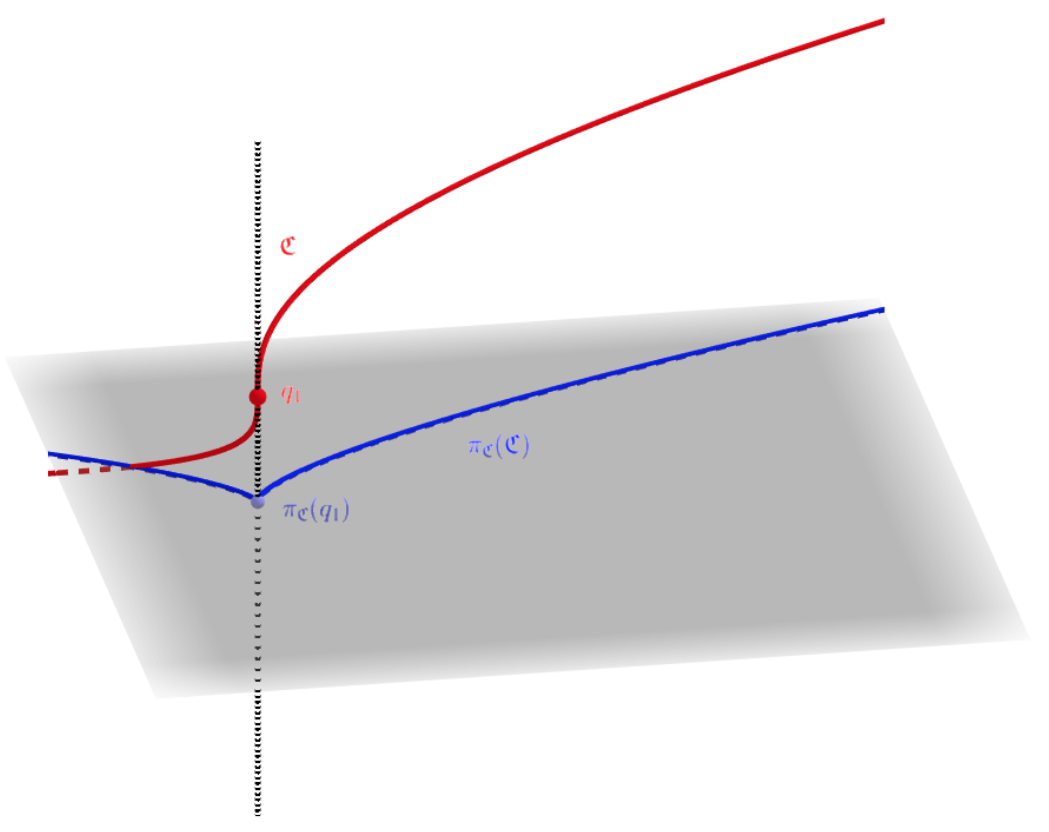

Figure 4: The curve $\mathfrak{C}$ (red) and its plane projection $\pi_{\mathfrak{C}}(\mathfrak{C})$ (blue) of Example 18 displaying a cusp singularity.

Example 18. Refer to Figure 4 Let $n=3$ and $B=\left\{\left(x_{1}, x_{2}, x_{3}\right) \in \mathbb{R}^{3} \mid x_{1}, x_{2}, x_{3} \in(-2,2)\right\}$. Define $P_{1}\left(x_{1}, x_{2}, x_{3}\right)=x_{1}-\left(x_{3}-1\right)^{3}, P_{2}\left(x_{1}, x_{2}, x_{3}\right)=x_{2}-\left(x_{3}-1\right)^{2}$ and $P=\left(P_{1}, P_{2}\right)$. The Jacobian matrix of $P$ has full rank over $\mathfrak{C}$, thus Assumption $\mathcal{A}_{1}$ is satisfied. The set $\mathfrak{L}_{n}$ is empty since $\pi_{\mathfrak{C}}$ is injective over $\mathfrak{C}$, hence Assumption $\mathcal{A}_{4}$ is satisfied. The only point of $\mathfrak{C}$ with a tangent line orthogonal to the $\left(x_{1}, x_{2}\right)$-plane is $q_{1}=(0,0,1)$, thus $\mathfrak{L}_{\mathrm{c}}=\left\{q_{1}\right\}$ and Assumption $\mathcal{A}_{2}$ is satisfied. By Lemma 23 the multiplicity of the system $\left\{P=0,\left(x_{1}, x_{2}\right)=\pi_{\mathfrak{C}}\left(q_{1}\right)\right\}$ at its unique solution $q_{1}$ is $\min \left\{\operatorname{mult}_{1}\left(\left(x_{3}-1\right)^{3}\right)\right.$, $\left.\operatorname{mult}_{1}\left(\left(x_{3}-1\right)^{2}\right)\right\}=\min \{3,2\}=$ 2 (mult is defined in Definition 2). Moreover, for any point $q_{0} \in \mathfrak{C}$ different from $q_{1}$, the multiplicity of the 


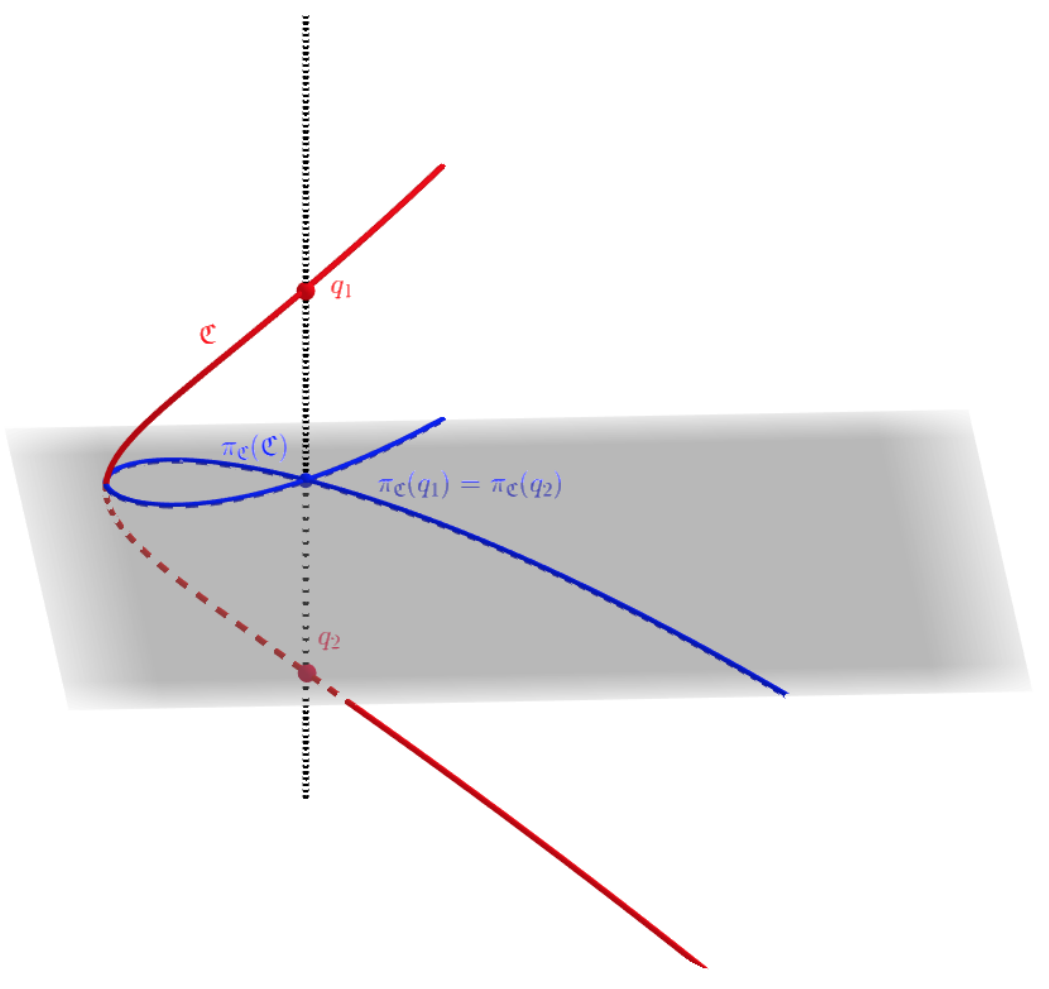

Figure 5: The curve $\mathfrak{C}\left(\right.$ red) and its plane projection $\pi_{\mathfrak{C}}(\mathfrak{C})$ (blue) of Example 19 displaying a node singularity.

corresponding system at its unique solution $q_{0}$ is one, thus $P$ satisfies Assumption $\mathcal{A}_{3}$ The system $\operatorname{Ball}(P)$ :

$$
\left\{\begin{array}{l}
x_{1}-3 r^{2} t y+3 r^{2} t-y^{3}+3 y^{2}-3 y+1=0 \\
x_{2}-r^{2} t-y^{2}+2 y-1=0 \\
-r^{3} t-3 r y^{2}+6 r y-3 r=0 \\
-2 r y+2 r=0 \\
r^{2}-1=0
\end{array}\right.
$$

Example 19. Refer to Figure 5 Let $B$ be defined as in Example 18 Define the functions $P_{1}\left(x_{1}, x_{2}, x_{3}\right)=x_{1}-$ $\left(x_{3}^{2}-1\right), P_{2}\left(x_{1}, x_{2}, x_{3}\right)=x_{2}-\left(x_{3}^{3}-x_{3}\right)$ and $P=\left(P_{1}, P_{2}\right)$. The Jacobian matrix of $P$ has full rank over $\mathfrak{C}$, thus Assumption $\mathcal{A}_{1}$ is satisfied. Moreover, the set $\mathfrak{L}_{\mathrm{c}}$ is empty and $\mathfrak{L}_{\mathrm{n}}=\left\{q_{1}, q_{2}\right\}$, with $q_{1}=(0,0,1), q_{2}=(0,0,-1)$, i.e., Assumptions $\mathcal{A}_{2}$ and $\mathcal{A}_{4}$ are satisfied. The multiplicity of the system $\left\{P=0 \in \mathbb{R}^{n-1}, x_{1}=x_{2}=0\right\}$ at both 
$q_{1}, q_{2}$ is equal to one, thus Assumption $\mathcal{A}_{3}$ is also satisfied. The system $\operatorname{Ball}(P)$ :

$$
\left\{\begin{array}{l}
x_{1}-r^{2} t-y^{2}+1=0 \\
x_{2}-r^{2} t y-y^{3}+y=0 \\
-2 r y=0 \\
-r^{3} t-3 r y^{2}+r=0 \\
r^{2}-1=0
\end{array}\right.
$$

has two twin solutions $X=(0,0,0,1,1)$ and $X^{\prime}=(0,0,0,-1,1)$ in $\mathbb{R}^{5}$ such that $\Omega_{P}(X)=\Omega_{P}\left(X^{\prime}\right)=$ $\left(q_{1}, q_{2}\right) \in \widehat{\mathfrak{L}}_{\mathrm{n}}$.

\subsection{Singularities induced by $\mathfrak{L}_{n}$}

We now study the types of singularities of the plane curve $\pi_{\mathfrak{C}}(\mathfrak{C})$ obtained by projecting points in $\mathfrak{L}_{\mathrm{n}}$, that is when several points of $\mathfrak{C}$ project to the same point. We begin by showing that the geometric property that the curve $\mathfrak{C}$ has a tangent orthogonal to the projection plane has an algebraic equivalent in terms of multiplicity.

Lemma 20. Let $P=\left(P_{1} \ldots, P_{n-1}\right) \in C^{\infty}\left(\mathbb{R}^{n}, \mathbb{R}^{n-1}\right)$ satisfy Assumption $\mathcal{A}_{1}$ Let $q$ be in $\overline{\mathfrak{C}}$ such that the multiplicity of the system $S=\left\{P(x)=0 \in \mathbb{R}^{n-1}, x_{1}-\alpha=x_{2}-\beta=0\right\}$ at $q$ is finite, where $(\alpha, \beta)=\pi_{\overline{\mathfrak{C}}}(q) \in \mathbb{R}^{2}$. Then, $q \in \overline{\mathfrak{L}_{\mathrm{c}}}$ if and only if the multiplicity of the system $S$ at $q$ is at least two.

Proof. Without loss of generality assume that $q=0 \in \mathbb{R}^{n}$.

Sufficiency: Assume that $q \in \overline{\mathfrak{L}_{\mathrm{c}}}$. Let $v=\left(v_{1}, \ldots, v_{n}\right)$ be a non-trivial vector of the tangent line of $\overline{\mathfrak{C}}$ at $q$. Thus, $J_{P}(q) \cdot v^{T}=0$. By the definition of $\overline{\mathfrak{L}_{\mathrm{c}}}$ we have $v_{1}=v_{2}=0$. Define the differential operator $c=\sum_{i=3}^{n} v_{i} \frac{\partial}{\partial x_{i}}$. Notice that $c \cdot P_{j}=\sum_{i=3}^{n} v_{i} \frac{\partial P_{j}}{\partial x_{i}}(q)=0$ for all integers $1 \leqslant j \leqslant n-1$ (see [DLZ11, 2.1] for the definition of $c \cdot P_{j}$ ). Moreover, by the definition of $c$ and since $v_{1}=v_{2}=0$, we have $c \cdot\left(x_{1}\right)=c \cdot\left(x_{2}\right)=0$. Hence, $c \in D_{q}^{1}[S] \backslash D_{q}^{0}[S]$. Thus, $\operatorname{dim}\left(D_{q}^{1}\right)>1$. Hence, the multiplicity of $S$ at $q$ is at least two.

Necessity: Assume that the multiplicity of $S$ at $q$ is at least two, then $D_{q}^{0}[S] \subsetneq D_{q}^{1}[S]$. This implies that there exists a non-trivial differential operator $c=\sum_{i=1}^{n} c_{i} \frac{\partial}{\partial x_{i}} \in D_{q}^{1}[S] \backslash D_{q}^{0}[S]$ such that:

(a) We have that $c \cdot P_{j}=0$ for all integers $1 \leqslant j \leqslant n-1$ which implies that if we write $v_{i}=c_{i}$, with $1 \leqslant i \leqslant n$, the non-trivial vector $v$ is in the tangent space of $\overline{\mathfrak{C}}$ at $q$.

(b) We have that $c \cdot\left(x_{1}\right)=c \cdot\left(x_{2}\right)=0$, equivalently, $c_{1}=c_{2}=0$. Thus, $v_{1}=v_{2}=0$.

The tangent line to the curve at $q$ is thus orthogonal to the $\left(x_{1}, x_{2}\right)$-plane. Thus, $q \in \overline{\mathfrak{L}_{\mathrm{c}}}$.

Lemma 21. Under Assumptions $\mathcal{A}_{1}, \mathcal{A}_{2}, \mathcal{A}_{3}$ and $\mathcal{A}_{4}$, if $q \in \mathfrak{L}_{\mathrm{n}}$, then $\pi_{\mathfrak{C}}(q)$ is a singular point of the plane curve $\pi_{\mathfrak{C}}(\mathfrak{C})$. More precisely, either $\pi_{\mathfrak{C}}(q)$ is of type $A_{2 k+1}^{-}$with $k \geqslant 0$, or there exists a non-null smooth function $g$ defined in a neighborhood of $0 \in \mathbb{R}$ with $\operatorname{mult}(g)=\infty$ such that $\left(\pi_{\mathfrak{C}}(q), \pi_{\mathfrak{C}}(\mathfrak{C})\right.$ ) is equivalent (according to Definition 6) to the curve defined by $x^{2}-g\left(y^{2}\right)=0$ at the origin. 
Proof. Let $p=\pi_{\mathfrak{C}}(q)$, according to $\mathcal{A}_{3}, \pi_{\mathfrak{C}}^{-1}(p)$ has at most two points, and since $q$ is in $\mathfrak{L}_{\mathrm{n}}$, it also has at least two points. Define $q^{\prime}$ such that $\pi_{\mathfrak{C}}^{-1}(p)=\left\{q, q^{\prime}\right\}$ and denote the plane curve $\pi_{\mathfrak{C}}(\mathfrak{C})$ by $C$. Without loss of generality, one can assume that $p=(0,0)$. In addition, $\mathcal{A}_{3}$ also implies that the multiplicities of $q$ and $q^{\prime}$ in the system $\left\{P(x)=0 \in \mathbb{R}^{n-1}, x_{1}=x_{2}=0\right\}$ are one. With Assumption $\mathcal{A}_{1}$ Lemma 20 then implies that the tangents to $\mathfrak{C}$ at $q$ and $q^{\prime}$ are not orthogonal to the $\left(x_{1}, x_{2}\right)$-plane. Thus there exists two neighborhoods $N_{q}$ and $N_{q^{\prime}}$ of $q$ and $q^{\prime}$ in $\mathbb{R}^{n}$ such that $\pi$ restricted to $\mathfrak{C} \cap N_{q}$ (resp. $\mathfrak{C} \cap N_{q^{\prime}}$ ) is an embedding. Let $D_{k}$ be a sequence of open disks centered at $p$ and of radius $\frac{1}{k}$. By contradiction, if for all $k$, there exists points $q_{k} \in \mathfrak{C}$ such that $q_{k}$ is not in $N_{q} \cup N_{q^{\prime}}$ and $\pi_{\mathfrak{C}}\left(q_{k}\right) \in D_{k}$, then the limit $q_{\infty}$ is a point of $\overline{\mathfrak{C}}$ distinct from $q$ and $q^{\prime}$, and $\pi_{\mathfrak{C}}\left(q_{\infty}\right)=p$. If $q_{\infty}$ is in $B$, it contradicts $\mathcal{A}_{3}$ and if it is in $\bar{B}$, it contradicts $\mathcal{A}_{4}$ Thus for a small enough neighborhood of $p$, the projection of the curve is restricted to the projection of the two branches around $q$ and $q^{\prime}$. Finally, if for all $D_{k}$, the pre-image of $\pi^{-1}\left(D_{k}\right)$ contains a point in $\overline{\mathfrak{L}_{\mathrm{n}}} \backslash\left\{q, q^{\prime}\right\}$, then this contradicts the discreteness assumption $\mathcal{A}_{4}$ Thus there exists a neighborhood $N \subseteq \mathbb{R}^{2}$ of $p$ such that $\pi_{\mathfrak{C}}^{-1}(N)$ is a union of two smooth (Assumption $\mathcal{A}_{1}$ open subsets of $\mathfrak{C}$ such that $q$ is on one branch and $q^{\prime}$ on the other, and $\pi_{\mathfrak{C}}$ restricted to $\pi_{\mathfrak{C}}^{-1}(N) \backslash\left\{p, q^{\prime}\right\}$ is an embedding. The projection of these two smooth branches are thus two smooth curves in the plane. Let these two smooth plane branches be defined by the zero sets of the smooth functions $f_{1}$ and $f_{2}$ in $C^{\infty}\left(\mathbb{R}^{2}, \mathbb{R}\right)$. Let $u$ (resp. $u^{\prime}$ ) be a non-zero tangent vector of $\mathfrak{C}$ at $q\left(\right.$ resp. $q^{\prime}$ ) and $v$ (resp. $v^{\prime}$ ) be its projection in $\mathbb{R}^{2}$. We distinguish two cases:

(a) The vectors $v$ and $v^{\prime}$ are independent in $\mathbb{R}^{2}$. Thus, $v$ and $v^{\prime}$ give rise to a local coordinate system $(x, y)$ in a neighborhood of $p$ in $\mathbb{R}^{2}$. The vector $v$ being tangent to the zero set of $f_{1}$, one has $\frac{\partial f_{1}}{\partial x}(p)=0$ and $\frac{\partial f_{1}}{\partial y}(p) \neq 0$. By the implicit function theorem [Dem00, Corollary 2.7.3.], we deduce that there exists a real smooth function $h_{1}$ such that $y=x^{2} \cdot h_{1}(x)$ is a local parameterization of the zero set of $f_{1}$. Similarly, there exists a smooth function $h_{2}$ such that $x=y^{2} \cdot h_{2}(y)$ is a local parameterization of the zero set of $f_{2}$. Thus $(x, y) \in N$ iff $f(x, y)=f_{1}(x, y) f_{2}(x, y)=0$ iff $\left(y-x^{2} \cdot h_{1}(x)\right)\left(x-y^{2} \cdot h_{2}(y)\right)=0$, equivalently, $\left[y-x-x^{2} \cdot h_{1}(x)+y^{2} \cdot h_{2}(y)\right]^{2}-\left[y+x-x^{2} \cdot h_{1}(x)-y^{2} \cdot h_{2}(y)\right]^{2}=0$. The change of coordinates $X=y-x+x^{2} \cdot h_{1}(x)+y^{2} \cdot h_{2}(y)$ and $Y=y+x+x^{2} \cdot h_{1}(x)-y^{2} \cdot h_{2}(y)$ is a diffeomorphism since $\operatorname{det}\left(J_{x, y}(X, Y)\right)_{p} \neq 0$. Then, the local equation of the curve $C$ at $p$ is of the form $X^{2}-Y^{2}$ with these new coordinates, which means that $p$ is a $A_{1}^{-}$or node singularity.

(b) The vectors $v$ and $v^{\prime}$ are co-linear. Then, choose $v^{\prime \prime} \in T_{p} \mathbb{R}^{2}$ linearly independent from $v$, the vectors $v, v^{\prime \prime}$ give rise to a coordinate system $(x, y)$ at $p$. In this coordinate system, we thus have $\frac{\partial f_{1}}{\partial x}(p)=\frac{\partial f_{2}}{\partial x}(p)=0$, $\frac{\partial f_{1}}{\partial y}(p) \neq 0$ and $\frac{\partial f_{2}}{\partial y}(p) \neq 0$. By the implicit function theorem, there exist smooth functions $h_{1}$ and $h_{2}$ such that locally $f(x, y)=0$ if and only if $\left(y-x^{2} \cdot h_{1}(x)\right)\left(y-x^{2} \cdot h_{2}(x)\right)=0$. The last equality is equivalent to $\left(2 y-x^{2}\left(h_{1}(x)+h_{2}(x)\right)\right)^{2}-x^{4}\left(h_{1}(x)-h_{2}(x)\right)^{2}=0$. Assumption $\mathcal{A}_{4}$ ensures that the projections of the two branches have only one common point, such that $h_{1}(x)-h_{2}(x)$ does not vanish identically. We distinguish two cases:

(i) $\operatorname{mult}\left(h_{1}(x)-h_{2}(x)\right)=k \leqslant \infty$, then $h_{1}(x)-h_{2}(x)=x^{k} \cdot u$ with $u(p) \neq 0$ and without loss 
Proof. Since $\operatorname{rank}\left(J_{P}(q)\right)=n-1$ (Assumption $\underline{\mathcal{A}_{1}}$, there exists $k \in\{1, \ldots, n\}$ such that $\operatorname{det}\left(M_{k}(q)\right) \neq 0$, where $M_{k}$ is the minor of $J_{P}$ obtained by removing the $k$-th column. Notice that $k \notin\{1,2\}$, since $q \in \mathfrak{L}_{\mathrm{c}}$ implies that $\operatorname{det}\left(M_{1}(q)\right)=\operatorname{det}\left(M_{2}(q)\right)=0$. Without loss of generality, we assume that $k=n$. Using the implicit of generality, assume that $u(p)>0$. The change of coordinates $X=2 y-x^{2}\left(h_{1}(x)+h_{2}(x)\right)$ and $Y=x \cdot u^{\frac{1}{2+k}}$ is a diffeomorphism (notice that indeed $u^{\frac{1}{2+k}}$ is a smooth function around $p$ ). Then, the local equation of the curve $C$ at $p$ is of the form $X^{2}-Y^{(2 k+3)+1}$ with these new coordinates, which means that $p$ is a singularity of type $A_{2 k+3}^{-}$.

(ii) $\operatorname{mult}\left(h_{1}(x)-h_{2}(x)\right)=\infty$. Since the function $x^{4}\left(h_{1}(x)-h_{2}(x)\right)^{2}$ is even, by Theorem 49 , there exists a smooth function $g$ such that $x^{4}\left(h_{1}(x)-h_{2}(x)\right)^{2}=g\left(x^{2}\right)$. Thus, taking the diffeomorphism $X=2 y-x^{2}\left(h_{1}(x)+h_{2}(x)\right)$ and $Y=x$, we get the second case of the claim.

\subsection{Singularities induced by $\mathfrak{L}_{\mathrm{c}}$}

We now study the types of singularities of the plane curve $\pi_{\mathfrak{C}}(\mathfrak{C})$ obtained by projecting points in $\mathfrak{L}_{\mathrm{c}}$, that is when the tangent to $\mathfrak{C}$ is orthogonal to the projection plane. We start by locally parametrizing $\mathfrak{C}$ around a point in $\mathfrak{L}_{\mathrm{c}}$. This parameterization will ease the computation of $\operatorname{Ball}(P)$ and its Jacobian in Section 4 In the rest of this section and Section 4 , the analysis is simplified by translating relevant points or assuming the curve $\mathfrak{C}$ is parametrizable by a specific variable. On the other hand, in our algorithmic Section 5 , the input is not modifiable at all but every computation uses interval arithmetic. This implies that the exact coordinates of a point may not be known, instead we only compute with a box containing it and isolating it from other relevant points. The idea of our semi-algorithms is to check that some function does not vanish on such a box. This then implies that such a function does not vanish at the point this box contains. The theoretical analysis of this section can then be applied to the point to deduce the appropriate property without the knowledge of the exact location of that point.

Lemma 22. Let $P \in C^{\infty}\left(\mathbb{R}^{n}, \mathbb{R}^{n-1}\right)$. Let $q \in \mathfrak{L}_{\mathrm{c}}$ such that Assumption $\mathcal{A}_{1}$ is satisfied in a neighborhood of $q$ in B. Without loss of generality one can assume $q=0 \in \mathbb{R}^{n}$. Then there exist an invertible matrix $M$ of size $(n-1) \times(n-1)$ of smooth functions in a neighborhood of $q$ and smooth functions $f_{1}, f_{2}, f_{3}, \ldots, f_{n-1}$ defined in a neighborhood of $0 \in \mathbb{R}$, such that:

$$
\left(\begin{array}{c}
x_{1}-f_{1}\left(x_{n}\right) \\
x_{2}-f_{2}\left(x_{n}\right) \\
x_{3}-f_{3}\left(x_{n}\right) \\
\ldots \\
\ldots \\
x_{n-1}-f_{n-1}\left(x_{n}\right)
\end{array}\right)=M \cdot\left(\begin{array}{c}
P_{1} \\
P_{2} \\
P_{3} \\
\ldots \\
\ldots \\
P_{n-1}
\end{array}\right),
$$

with $\min \left\{\operatorname{mult}\left(f_{1}\left(x_{n}\right)\right)\right.$, mult $\left.\left(f_{2}\left(x_{n}\right)\right)\right\}>1$ (mult is defined in Definition 2). were $M_{k}$ is the minor of $J_{P}$ obtained by removing he $k$-h column. Notice that $k \notin\{1,2\}$, since $q \in \mathfrak{L}$ inplies 
function theorem [Corollary 2.7.3] [Dem00], there exist smooth functions $f_{1}, \ldots, f_{n-1}$ of one variable such that we have that

$$
P_{j}\left(f_{1}\left(x_{n}\right), \ldots, f_{n-1}\left(x_{n}\right), x_{n}\right)=0, j \in\{1, \ldots n-1\} .
$$

Define the function $\varphi$ that maps $x_{i}$ to $z_{i}=x_{i}-f_{i}\left(x_{n}\right)$, for all $i \in\{1, \ldots, n-1\}$ and $x_{n}$ to $z_{n}=x_{n}$. We can see that $\varphi$ is a diffeomorphism and $z=\left(z_{1}, \ldots, z_{n}\right)$ is a local coordinate system around $q$. Hence, we can define the function $G_{j}(z)=P_{j} \circ \varphi^{-1}(z)=P_{j}(x)$ for all integers $1 \leqslant j \leqslant n-1$. Using Hadamard's Lemma [Dem00, Proposition 4.2.3] for the first $n-1$ variables of $z$, we can write $G_{j}(z)-G_{j}\left(0, \ldots, 0, z_{n}\right)=$ $\sum_{i=1}^{n-1} z_{i} \cdot h_{j i}(z)$ for some smooth functions $h_{j i}$. Note that $\varphi^{-1}(z)=\left(z_{1}+f_{1}\left(z_{n}\right), \ldots, z_{n-1}+f_{n-1}\left(z_{n}\right), z_{n}\right)$. Hence, $G_{j}\left(0, \ldots, 0, z_{n}\right)=P_{j} \circ \varphi^{-1}\left(0, \ldots, 0, z_{n}\right)=P_{j}\left(f_{1}\left(z_{n}\right), \ldots, f_{n-1}\left(z_{n}\right), z_{n}\right)=P_{j}\left(f_{1}\left(x_{n}\right), \ldots, f_{n-1}\left(x_{n}\right), x_{n}\right)$. The latter function is equal to zero by (3.5). Thus, $P_{j}(x)=G_{j}(z)=\sum_{i=1}^{n-1} z_{i} \cdot h_{j i}(z)=\sum_{i=1}^{n-1}\left(x_{i}-f_{i}\left(x_{n}\right)\right) \cdot H_{j i}(x)$, with $H_{j i}(x)=h_{j i} \circ \varphi(x)$.

Defining $M_{0}=\left(H_{j i}\right)_{1 \leqslant j, i \leqslant n-1}$ we get:

$$
\left(\begin{array}{c}
P_{1} \\
\cdots \\
\cdots \\
P_{n-1}
\end{array}\right)=M_{0} \cdot\left(\begin{array}{c}
x_{1}-f_{1}\left(x_{n}\right) \\
\ldots \\
\cdots \\
x_{n-1}-f_{n-1}\left(x_{n}\right)
\end{array}\right) .
$$

Notice that $M_{0}$ evaluated at $q$ is the invertible matrix $M_{n}(q)$. Hence, by continuity of the determinant function, there is a neighborhood of $q$ in which $M_{0}$ is invertible. Thus, writing $M$ as the inverse of $M_{0}$ we get:

$$
Q_{0}=\left(\begin{array}{c}
x_{1}-f_{1}\left(x_{n}\right) \\
\cdots \\
\cdots \\
x_{n-1}-f_{n-1}\left(x_{n}\right)
\end{array}\right)=M \cdot\left(\begin{array}{c}
P_{1} \\
\cdots \\
\cdots \\
P_{n-1}
\end{array}\right) .
$$

To prove that $\min \left\{\operatorname{mult}\left(f_{1}\left(x_{n}\right)\right)\right.$, $\left.\operatorname{mult}\left(f_{2}\left(x_{n}\right)\right)\right\}>1$, we take the Jacobian matrices of both sides of 3.6 and we evaluate them at $q=0$. We get the equation $J_{Q_{0}}(q)=M(q) \cdot J_{P}(q)$. By invertibility of $M(q)$ we deduce that the $k$-th minors (obtained by removing the $k$-th column) of $J_{Q_{0}}(q)$ and $J_{P}(q)$ have the same rank. Computing $J_{Q_{0}}(q)$ and considering the fact that $\operatorname{det}\left(M_{1}(q)\right)=\operatorname{det}\left(M_{2}(q)\right)=0$ implies that $f_{1}^{\prime}(0)=f_{2}^{\prime}(0)=0$, we thus have that $\min \left\{\operatorname{mult}\left(f_{1}\left(x_{n}\right)\right), \operatorname{mult}\left(f_{2}\left(x_{n}\right)\right)\right\}$ is at least two.

Lemma 23. Preserving the notation and the assumptions in Lemma 22 the multiplicity $m$ of the system $S=$ $\left\{Q_{0}(x)=0 \in \mathbb{R}^{n-1}, x_{1}=x_{2}=0\right\}$ at $q$ is equal to $d=\min \left\{\operatorname{mult}\left(f_{1}\left(x_{n}\right)\right)\right.$, $\left.\operatorname{mult}\left(f_{2}\left(x_{n}\right)\right)\right\}$.

Proof. First, we start with the case $m<\infty$. By Proposition 5, we can assume without loss of generality, that $f_{1}, \ldots, f_{n-1}$ are polynomials. Following the notation in Definition 3 , let $\mathbb{R}[x]$ (resp. $\mathbb{R}\left[x_{n}\right]$ ) be the ring of polynomials with $n$ variables (resp. one variable) and $\mathbb{R}[x]_{q}$ (resp. $\mathbb{R}\left[x_{n}\right]_{0}$ ) be its localization at $q$ (resp. $0 \in \mathbb{R}$ ). Also, define $I_{S}$ to be the ideal generated by the polynomials of $S$ in $\mathbb{R}[x]_{q}$ (as $I_{G}$ is defined in Definition 3 , , 
Lemma 24. Let $P \in C^{\infty}\left(\mathbb{R}^{n}, \mathbb{R}^{n-1}\right)$. Let $q \in \mathfrak{L}_{\mathrm{c}}$ such that Assumptions $\mathcal{A}_{1}, \mathcal{A}_{2}, \mathcal{A}_{3}$ and $\mathcal{A}_{4}$ hold in a neigh-
borhood of $q$ in $B$, then there exist an invertible matrix $\widetilde{M}$ of size $(n-1) \times(n-1)$ of smooth functions in a

Lemma 24. Let $P \in C^{\infty}\left(\mathbb{R}^{n}, \mathbb{R}^{n-1}\right)$. Let $q \in \mathfrak{L}_{\mathrm{c}}$ such that Assumptions $\mathcal{A}_{1}, \mathcal{A}_{2}, \mathcal{A}_{3}$ and $\mathcal{A}_{4}$ hold in a neigh-
borhood of $q$ in $B$, then there exist an invertible matrix $\widetilde{M}$ of size $(n-1) \times(n-1)$ of smooth functions in a i.e., $I_{S}=\left\langle x_{1}-f_{1}\left(x_{n}\right), x_{2}-f_{2}\left(x_{n}\right), \ldots x_{n-1}-f_{n-1}\left(x_{n}\right), x_{1}, x_{2}\right\rangle=\left\langle x_{1}-f_{1}\left(x_{n}\right), x_{2}-f_{2}\left(x_{n}\right), \ldots x_{n-1}-\right.$ $\left.f_{n-1}\left(x_{n}\right), f_{1}\left(x_{n}\right), f_{2}\left(x_{n}\right)\right\rangle$. If $f_{1}\left(x_{n}\right)=f_{2}\left(x_{n}\right)=0$, then the ideal $I_{S}$ is of dimension one, hence, $S$ has an infinite number of solutions which contradicts the assumption that $m<\infty$. Thus, $d<\infty$ which means that there exist $h_{1}, h_{2} \in \mathbb{R}\left[x_{n}\right]_{0}$ such that $h_{1}\left(x_{n}\right) f_{1}\left(x_{n}\right)+h_{2}\left(x_{n}\right) f_{2}\left(x_{n}\right)=x_{n}^{d}$. Thus, $I_{S}=\left\langle x_{1}-f_{1}\left(x_{n}\right), x_{2}-\right.$ $\left.f_{2}\left(x_{n}\right), \ldots, x_{n-1}-f_{n-1}\left(x_{n}\right), x_{n}^{d}\right\rangle$. Note that the set $\left\{x_{1}-f_{1}\left(x_{n}\right), x_{2}-f_{2}\left(x_{n}\right), \ldots x_{n-1}-f_{n-1}\left(x_{n}\right), x_{n}^{d}\right\}$ is a Gröbner basis of $I_{S}$ with respect to Local Lexicographical ordering $x_{1}>\cdots>x_{n}$. Hence, By [CLO05, Theorem 4.4.3] we have $\operatorname{dim}\left(\frac{\mathbb{R}[x]_{q}}{I_{S}}\right)=\operatorname{dim}\left(\frac{\mathbb{R}[x]_{q}}{L T\left(I_{S}\right)}\right)=\operatorname{dim}\left(\frac{\mathbb{R}[x]_{q}}{\left\langle x_{1}, x_{2}, \ldots x_{n-1}, x_{n}^{d}\right\rangle}\right)$, where $L T\left(I_{S}\right)$ is the ideal generated by the leading terms of $I_{S}$. Consequently, $m=\operatorname{dim}\left(\frac{\mathbb{R}[x]_{q}}{I_{S}}\right)=\operatorname{dim}\left(\frac{\mathbb{R}\left[x_{n}\right]_{0}}{\left\langle x_{n}^{d}\right\rangle}\right)=d$.

Second, assume that $m=\infty$. We prove that $d=\infty$, that is, $\frac{\partial^{k} f_{1}}{\partial x_{n}^{k}}(0)=\frac{\partial^{k} f_{2}}{\partial x_{n}^{k}}(0)=0$ for any positive integer $k$. Preserving the notation in Definition 4 , consider the dual space $D_{q}^{k}[S]$. We are going to show that for any positive integer $k$ and any element $c \in D_{q}^{k}[S] \backslash D_{q}^{k-1}[S]$ (which always exists since $m=\infty$ ), the coefficient $c_{x_{n}^{k}}$ corresponding to $\frac{\partial^{k}}{\partial x_{n}^{k}}$, for $c$, is non-zero. We consequentially show that $\frac{\partial^{k} f_{1}}{\partial x_{n}^{k}}(0)=\frac{\partial^{k} f_{2}}{\partial x_{n}^{k}}(0)=0$. We prove the previous statements by induction on $k$.

For $k=1$, since $q \in \mathfrak{L}_{\mathrm{c}}$, we already showed in the proof of Lemma 20 that a non-trivial element $c=\sum_{i=1}^{n} v_{i} \frac{\partial}{\partial x_{i}}$ is in $D_{q}^{1}[S] \backslash D_{q}^{0}[S]$ if and only if $v=\left(v_{1}, \ldots, v_{n}\right)$ is in $T_{q} \mathfrak{C}$. On the other hand, $T_{q} \mathfrak{C}$ is generated by the vector $\left(f_{1}^{\prime}(0), \ldots f_{n-1}^{\prime}(0), 1\right)$, thus $c_{x_{n}^{1}}=v_{n} \neq 0$. The function $f_{1}\left(x_{n}\right)$ is in the set of functions generated by $S$ thus $0=c \cdot\left(f_{1}\left(x_{n}\right)\right)=\sum_{i=1}^{n} v_{i} \frac{\partial}{\partial x_{i}} q \cdot\left(f_{1}\left(x_{n}\right)\right)=c_{x_{n}^{1}} \frac{\partial f_{1}}{\partial x_{n}}(0)$, and thus $\frac{\partial f_{1}}{\partial x_{n}}(0)=0$. Thus, the induction hypothesis holds for $k=1$.

Define $c^{\prime}=\phi_{n}(c)$ and consider two cases:

(a) $c^{\prime} \in D_{q}^{k-1}[S] \backslash D_{q}^{k-2}[S]:$ By the induction hypothesis, the coefficient $c_{x_{n}^{k-1}}^{\prime}$ corresponding to $\frac{\partial^{k-1}}{\partial x_{n}^{k-1}}$ for $c^{\prime}$ is non-zero and $\frac{\partial^{k^{\prime}} f_{1}}{\partial x_{n}^{k^{\prime}}}(0)=\frac{\partial^{k^{\prime}} f_{2}}{\partial x_{n}^{k^{\prime}}}(0)=0$, for all $k^{\prime}<k$. Notice that by the definition of $\phi_{n}$, we have $c_{x_{n}^{k}}=c_{x_{n}^{k-1}}^{\prime} \neq 0$. Hence, $0=c \cdot f_{1}\left(x_{n}\right)=\sum_{i=1}^{k} c_{x_{n}^{i}} \frac{\partial^{i} f_{1}}{\partial x_{n}^{i}}(0)=c_{x_{n}^{k}} \frac{\partial^{k} f_{1}}{\partial x_{n}^{k}}(0)$. Hence, $\frac{\partial^{k} f_{1}}{\partial x_{n}^{k}}(0)=0$. Similarly, we prove that $\frac{\partial^{k} f_{2}}{\partial x_{n}^{k}}(0)=0$. Thus in Case (a), the lemma is proved.

(b) $c^{\prime} \in D_{q}^{k-2}[S]$ : Since $c \in D_{q}^{k}[S] \backslash D_{q}^{k-1}[S]$, there exists $j \in\{1, \ldots, n-1\}$ such that the element $c^{\prime \prime}=\phi_{j}(c)$ is in $D_{q}^{k-1}[S] \backslash D_{q}^{k-2}[S]$. By the induction hypothesis, the coefficient $c_{x_{n}^{k-1}}^{\prime \prime}$ corresponding to $\frac{\partial^{k-1}}{\partial x_{n}^{k-1}}$ for $c^{\prime \prime}$, is non-zero. On the other hand, $c_{x_{j} x_{n}^{k-1}}=c_{x_{n}^{k-1}}^{\prime \prime} \neq 0$. Hence, since $\phi_{n}\left(c_{x_{j} x_{n}^{k-1}} \frac{\partial^{k}}{\partial x_{j} \partial x_{n}^{k-1}}\right) \in D_{q}^{k-1}[S] \backslash$ $D_{q}^{k-2}[S]$, then so is $\phi_{n}(c)=c^{\prime}$ which contradicts the assumption. Thus, Case (b) is impossible.

With the additional Assumptions $\mathcal{A}_{2}, \mathcal{A}_{3}$ and $\mathcal{A}_{4}$, one can give a more precise form of $f_{1}$ and $f_{2}$ in Equation (3.4). 
neighborhood of $q$, a smooth diffeomorphism $\varphi$ defined in an open subset of $\mathbb{R}^{n}$, with $z=\left(z_{1}, \ldots, z_{n}\right)=\varphi^{-1}(x)$ and smooth functions $f_{3}, \ldots, f_{n-1}, g$ defined in a neighborhood of $0 \in \mathbb{R}$, such that

$$
Q=\left(\begin{array}{c}
z_{1}-z_{n} \cdot g\left(z_{n}^{2}\right) \\
z_{2}-z_{n}^{2} \\
z_{3}-f_{3}\left(z_{n}\right) \\
\ldots \\
\ldots \\
z_{n-1}-f_{n-1}\left(z_{n}\right)
\end{array}\right)=\widetilde{M} \cdot\left(\begin{array}{c}
P_{1} \\
P_{2} \\
P_{3} \\
\ldots \\
\ldots \\
P_{n-1}
\end{array}\right) \circ \varphi
$$

on a neighborhood of $q$. Moreover, either $\operatorname{mult}\left(g\left(z_{n}\right)\right)=\infty$ or there exists an integer $k>0$ with $g\left(z_{n}\right)=z_{n}^{k}$.

Proof. Step 1: Equation (3.6) implies that $Q_{0}$ and $P$ define the same curve $\mathfrak{C}$ in a neighborhood of $q$ and that the function $Q_{0}$ satisfies the same assumptions as $P$ around $q$. By Lemma $23, d=\min \left\{\operatorname{mult}\left(f_{1}\left(x_{n}\right)\right), \operatorname{mult}\left(f_{2}\left(x_{n}\right)\right)\right\}$ is the multiplicity of the system $\left\{Q_{0}(x)=0 \in \mathbb{R}^{n-1}, x_{1}=0, x_{2}=0\right\}$ at $q$. By Assumption $\mathcal{A}_{3}$, we have that $d=2$.

Without loss of generality, assume that $\operatorname{mult}\left(f_{2}\left(x_{n}\right)\right)=2$ and $\frac{\partial^{2} f_{2}}{\partial x_{n}^{2}}(0)=2$. Hence, there is a smooth function $v$ such that $f_{2}\left(x_{n}\right)=x_{n}^{2}\left(1+x_{n} \cdot v\left(x_{n}\right)\right)$. Now, consider the diffeomorphism $\phi_{n}$ that sends $x_{n}$ to $z_{n}=x_{n} \sqrt{1+x_{n} \cdot v\left(x_{n}\right)}$. We have that $x_{2}-f_{2}\left(x_{n}\right)=x_{2}-z_{n}^{2}$. Define $\tilde{f}_{1}\left(z_{n}\right)=f_{1}\left(\phi_{n}^{-1}\left(z_{n}\right)\right)$ and $\tilde{f}_{2}\left(z_{n}\right)=f_{2}\left(\phi_{n}^{-1}\left(z_{n}\right)\right)=z_{n}^{2}$. Since $\operatorname{mult}\left(\tilde{f}_{1}\left(z_{n}\right)\right)=\operatorname{mult}\left(f_{1}\left(x_{n}\right)\right) \geqslant d=2$, there exists a smooth function $h$ such that $\tilde{f}_{1}\left(z_{n}\right)=z_{n}^{2} h\left(z_{n}\right)$. Write $\tilde{f}_{1}\left(z_{n}\right)=z_{n}^{2}\left[\frac{h\left(z_{n}\right)+h\left(-z_{n}\right)}{2}+\frac{h\left(z_{n}\right)-h\left(-z_{n}\right)}{2}\right]$. Since $\frac{h\left(z_{n}\right)+h\left(-z_{n}\right)}{2}$ (resp. $\left.\frac{h\left(z_{n}\right)+h\left(-z_{n}\right)}{2}\right)$ is even (resp. odd), then by Theorem 49 there exists a smooth function $\xi_{1}$ (resp. $\xi_{2}$ ) such that $\frac{h\left(z_{n}\right)+h\left(-z_{n}\right)}{2}=\xi_{1}\left(z_{n}^{2}\right)$ (resp. $\left.\frac{h\left(z_{n}\right)-h\left(-z_{n}\right)}{2}=z_{n} \xi_{2}\left(z_{n}^{2}\right)\right)$. Thus, $\tilde{f}_{1}\left(z_{n}\right)=z_{n}^{2}\left(\xi_{1}\left(z_{n}^{2}\right)+z_{n} \xi_{2}\left(z_{n}^{2}\right)\right)$. Notice that $\xi_{2}\left(x_{n}^{2}\right)$ cannot be the zero function, otherwise $\tilde{f}_{1}(\epsilon)=\tilde{f}_{1}(-\epsilon)$ and $\tilde{f}_{2}(\epsilon)=\tilde{f}_{2}(-\epsilon)$ for all small enough $\epsilon>0$, which contradicts Assumption $\mathcal{A}_{4}$

Step 2: We have two cases:

Case 1: $\operatorname{mult}\left(\xi_{2}\left(z_{n}\right)\right)=\infty$, then define the diffeomorphism $\phi$ which sends $x_{1}$ to $z_{1}=x_{1}-x_{2} \xi_{1}\left(x_{2}\right), x_{i}$ to $z_{i}=x_{i}$ for all integers $i \in\{2, \ldots, n-1\}$ and $x_{n}$ to $z_{n}=x_{n} \sqrt{1+x_{n} \cdot v\left(x_{n}\right)}$. Taking $g\left(z_{n}\right)=z_{n} \xi_{2}\left(z_{n}\right)$ and $\varphi=\phi^{-1}$ we prove the claim for the first case.

Case 2: $\operatorname{mult}\left(\xi_{2}\left(z_{n}\right)\right)=k<\infty$, that is, $\xi_{2}\left(z_{n}\right)=z_{n}^{k} u\left(z_{n}\right)$, for some smooth function $u$, with $u(0) \neq 0$ and an integer $k \geqslant 0$. Hence, we can write $x_{1}-\tilde{f}_{1}\left(z_{n}\right)=x_{1}-z_{n}^{2} \xi_{1}\left(z_{n}^{2}\right)-z_{n}^{2 k+3} u\left(z_{n}^{2}\right)=x_{1}-x_{2} \xi_{1}\left(x_{2}\right)-z_{n}^{2 k+3} u\left(x_{2}\right)$.

So, defining the diffeomorphism $\phi$ which sends $x_{i}$ to $z_{i}=x_{i}$ for all integers $i \in\{2, \ldots, n-1\}, x_{n}$ to $z_{n}=$ $x_{n} \sqrt{1+x_{n} \cdot v\left(x_{n}\right)}$ and $x_{1}$ to $z_{1}=\left(x_{1}-x_{2} \xi_{1}\left(x_{2}\right)\right) u^{-1}\left(x_{2}\right)$ (which means that $x_{1}-f_{1}\left(x_{n}\right)=u\left(x_{2}\right)\left[z_{1}-z_{n}^{2 k+3}\right]$ ), we get that: 


$$
\left(\begin{array}{c}
x_{1}-f_{1}\left(x_{n}\right) \\
\ldots \\
\ldots \\
x_{n-1}-f_{n-1}\left(x_{n}\right)
\end{array}\right)=\left(\begin{array}{cc}
u\left(x_{2}\right) & 0_{1 \times(n-2)} \\
0_{(n-2) \times 1} & I_{n-2}
\end{array}\right) \cdot\left(\begin{array}{c}
z_{1}-z_{n}^{2 k+3} \\
z_{2}-z_{n}^{2} \\
z_{3}-f_{3}\left(z_{n}\right) \\
\ldots \\
\ldots \\
z_{n-1}-f_{n-1}\left(z_{n}\right)
\end{array}\right) \circ \phi
$$

for a small enough neighborhood of $q$, where $I_{n-2}$ is the identity matrix of size $n-2$. Comparing with [3.4], we get:

$$
M \cdot\left(\begin{array}{c}
P_{1} \\
P_{2} \\
P_{3} \\
\cdots \\
\cdots \\
P_{n-1}
\end{array}\right)=\left(\begin{array}{cc}
u\left(x_{2}\right) & 0_{1 \times(n-2)} \\
0_{(n-2) \times 1} & I_{n-2}
\end{array}\right) \cdot\left(\begin{array}{c}
z_{1}-z_{n}^{2 k+3} \\
z_{2}-z_{n}^{2} \\
z_{3}-f_{3}\left(z_{n}\right) \\
\ldots \\
\ldots \\
z_{n-1}-f_{n-1}\left(z_{n}\right)
\end{array}\right) \circ \phi
$$

Hence, taking $\widetilde{M}=\left(\begin{array}{cc}u\left(x_{2}\right) & 0_{1 \times(n-2)} \\ 0_{(n-2) \times 1} & I_{n-2}\end{array}\right)^{-1} \cdot M$ and $\varphi=\phi^{-1}$ we recover 3.7.

Following the conclusion of Lemma 24, the reader may wonder whether the projection of $q$ in $\pi_{\mathfrak{C}}$ is always singular. This is clear when $g\left(x_{n}\right)=x_{n}^{k}$ for $0<k<\infty$ since this implies $z_{1}^{2}-z_{2}^{k+1}=0$ and thus $\pi_{\mathfrak{C}}(q)$ is a singularity of the type $A_{2 k}$. We next prove that the projection is also singular if $\operatorname{mult}\left(g\left(z_{n}\right)\right)=\infty$.

Lemma 25. Preserving the notation and the assumptions in Lemma 24 consider the function g defined in 3.7 , if $\operatorname{mult}\left(g\left(z_{n}\right)\right)=\infty$, then $\pi_{\mathfrak{C}}(q)$ is singular in $\pi_{\mathfrak{C}}(\mathfrak{C})$.

Proof. Since mult $\left(g\left(z_{n}\right)\right)=\infty$, then Case 1 in the proof of Lemma 24 holds. Moreover, we saw in the same proof that $\xi_{2}\left(z_{n}^{2}\right)$ (restricted to an open neighborhood of $0 \in \mathbb{R}$ ) cannot be the zero function. This implies that neither is the function $g\left(z_{n}^{2}\right)=z_{n}^{2} \xi\left(z_{n}^{2}\right)$, i.e., $g\left(z_{n}^{2}\right)$, restricted to an open neighborhood of $0 \in \mathbb{R}$, is not the zero function. Assume for the sake of contradiction that $\pi_{\mathfrak{C}}(q)$ is smooth in $\pi_{\mathfrak{C}}(\mathfrak{C})$, then using the implicit function theorem, there exists a $C^{\infty}$-function defined in a neighborhood of 0 in $\mathbb{R}$, with $f(0)=0$ such that for a small neighborhood of $\pi_{\mathfrak{C}}(q)$ in $\mathbb{R}^{2}$, one of the following cases is satisfied:

(a) $f\left(z_{1}\right)=z_{2} \Longleftrightarrow\left(z_{1}, z_{2}\right) \in \pi_{\mathfrak{C}}(\mathfrak{C})$. Then, by 3.7), we have $f\left(z_{n} g\left(z_{n}^{2}\right)\right)=z_{n}^{2}$. Taking the second derivative of both sides with respect to $z_{n}$ and then evaluating at 0 (recall that mult $\left(g\left(z_{n}\right)\right)=\infty$ ), we get the contradiction $0=2$.

(b) $f\left(z_{2}\right)=z_{1} \Longleftrightarrow\left(z_{1}, z_{2}\right) \in \pi_{\mathfrak{C}}(\mathfrak{C})$. Then $f\left(z_{n}^{2}\right)=z_{n} g\left(z_{n}^{2}\right)$. The function $z_{n} g\left(z_{n}^{2}\right)$ is an odd function but not the zero function, and on the other hand $f\left(z_{2}\right)$ is an even function, which leads to a contradiction. 
Thus, in both cases we have a contradiction, that is, $f$ does not exist and $\pi_{\mathfrak{C}}(q)$ cannot be smooth in $\pi_{\mathfrak{C}}(\mathfrak{C})$.

Returning to (3.7), notice that $\varphi$ is defined in such a way that it preserves the singularity class of $\pi_{\mathfrak{C}}(\mathfrak{C})$ at the point $\pi_{\mathfrak{C}}(q)$. In other words, if $C$ is the plane projection of the curve defined by $Q$ then $\left(\pi_{\mathfrak{C}}(\mathfrak{C}), 0\right)$ and $(C, 0)$ are equivalent.

\subsection{Proof of Theorem 11}

We first characterize the singularities of the projected curve $\pi_{\mathfrak{C}}(\mathfrak{C})$ by the points in $\mathfrak{L}_{n}$ and $\mathfrak{L}_{\mathrm{c}}$. The proof of Theorem 11 is then obtained via the bijection $\Omega_{P}$ (Definition 14 ) between $\widehat{\mathfrak{L}}$ and the solutions of the Ball system.

Lemma 26. If $P$ satisfies Assumptions $\mathcal{A}_{1}, \mathcal{A}_{2}, \mathcal{A}_{3}$ and $\mathcal{A}_{4}$ then a point $q \in \mathfrak{C}$ projects to a singular point in $\pi_{\mathfrak{C}}(\mathfrak{C})$ if and only if $q \in \mathfrak{L}_{\mathrm{c}} \cup \mathfrak{L}_{\mathrm{n}}$.

Proof. If $q \in \mathfrak{L}_{\mathrm{c}} \cup \mathfrak{L}_{\mathrm{n}}$, then by Lemmas 21, 24, and 25, $\pi_{\mathfrak{C}}(q)$ is singular in $\pi_{\mathfrak{C}}(\mathfrak{C})$. Conversely, if $q \notin \mathfrak{L}_{\mathrm{c}} \cup \mathfrak{L}_{\mathrm{n}}$, we prove that $\pi_{\mathfrak{C}}(q)$ is smooth in $\pi_{\mathfrak{C}}(\mathfrak{C})$.

Since $q \notin \mathfrak{L}_{\mathfrak{c}}$, the plane projection of $T_{q} \mathfrak{C}$ is a line, or equivalently, the derivative $T_{q} \pi_{\mathfrak{C}}$ of $\pi_{\mathfrak{C}}$ at $q$ is injective. Thus, $\pi_{\mathfrak{C}}$ is an immersion at $q$ ([Dem00, Definition 2.9.3]). Hence, for a small enough neighborhood $U_{0}$ of $q$ in $\mathbb{R}^{n}$, we have that $\pi_{\mathfrak{C}}$ restricted to $V=U_{0} \cap \mathfrak{C}$ is an embedding (see [Dem00, Proposition 2.9.6]). We are going to prove that, assuming that $U_{0}$ is small enough, the curve $\pi_{\mathfrak{C}}(\mathfrak{C})$ has exactly one branch around $\pi_{\mathfrak{C}}(q)$ which implies that $\pi_{\mathfrak{C}}(\mathfrak{C})$ is smooth at $\pi_{\mathfrak{C}}(q)$ since $\mathfrak{C}$ is smooth at $q$ by Assumption $\mathcal{A}_{1}$

To prove this claim, assume that there exists an open subset $U_{0}^{\prime}$ in $\mathbb{R}^{n}$ such that the set $V^{\prime}=U_{0}^{\prime} \cap \mathfrak{C}$ and $V$ are disjoint, but $\pi_{\mathfrak{C}}(q)$ is in the closure of $\pi_{\overline{\mathfrak{C}}}\left(V^{\prime}\right)$. Let $q_{k}$ be a sequence of points in $V^{\prime}$ such that $\pi_{\mathfrak{C}}\left(q_{k}\right)$ converges to $\pi_{\mathfrak{C}}(q)$. Since $\bar{B}$ is compact, there exists a convergent sub-sequence of $q_{k}$ that has a limit $q^{\prime}$ in $\bar{B}$. Notice that $\pi_{\overline{\mathfrak{C}}}\left(q^{\prime}\right)=\pi_{\mathfrak{C}}(q)$ by the continuity of $\pi_{\overline{\mathfrak{C}}}$. Hence, $q, q^{\prime}$ are both in $\overline{\mathfrak{L}_{\mathrm{n}}}$. However, since $q \notin \mathfrak{L}_{\mathrm{n}}$, we must have that $q^{\prime} \notin B$. Hence, $q^{\prime}$ is in the boundary of $B$ which contradicts Assumption $\mathcal{A}_{4}$. Hence, the curve $\pi_{\mathfrak{C}}(\mathfrak{C})$ has exactly one smooth branch around $\pi_{\mathfrak{C}}(q)$ which concludes the proof.

Finally, we prove that the solutions of the Ball system project to the singular points of $\pi_{\mathfrak{C}}(\mathfrak{C})$.

Proof of Theorem 117. By Lemma 26, if $\left(x_{1}, x_{2}\right)$ is singular in $\pi_{\mathfrak{C}}(\mathfrak{C})$, then there exists a point $q_{1} \in \mathfrak{L}_{\mathrm{c}} \cup \mathfrak{L}_{\mathrm{n}}$, with $\pi_{\mathfrak{C}}\left(q_{1}\right)=\left(x_{1}, x_{2}\right)$. If $q_{1} \in \mathfrak{L}_{\mathrm{c}}$, let $q_{2}=q_{1}$ and otherwise let $q_{2}$ be the unique (by Assumption $\mathcal{A}_{3}$ point in $\mathfrak{L}_{\mathrm{n}}$, distinct from $q_{1}$, that projects onto $\left(x_{1}, x_{2}\right)$, i.e. $\pi_{\mathfrak{C}}\left(q_{1}\right)=\pi_{\mathfrak{C}}\left(q_{2}\right)=\left(x_{1}, x_{2}\right)$. Hence, $\left(q_{1}, q_{2}\right)$ is in $\widehat{\mathfrak{L}}$. Since $\Omega_{P}$ is surjective (Lemma 15), there exists $X=\left(x_{1}, x_{2}, y, r, t\right) \in \operatorname{Sol}_{\operatorname{Ball}(P)}$ with $\Omega_{P}(X)=\left(q_{1}, q_{2}\right)$.

On the other hand, if $X$ is a solution of $\operatorname{Ball}(P)$, then by Lemma 13 the pair $\left(q_{1}, q_{2}\right)=\Omega_{P}(X)$ is in $\widehat{\mathfrak{L}}$. Hence, $q_{1}=\left(x_{1}, x_{2}, y+r \sqrt{t}\right) \in \mathbb{R} \times \mathbb{R} \times \mathbb{R}^{n-2}$ is in $\mathfrak{L}_{\mathrm{c}} \cup \mathfrak{L}_{\mathrm{n}}$. Hence, by Lemma 26 the point $\left(x_{1}, x_{2}\right)$ is singular in $\pi_{\mathfrak{C}}(\mathfrak{C})$. 


\section{Regularity of the Ball system}

In this section, our goal is to prove Theorem 27 determining necessary and sufficient conditions for Ball $(P)$ to be regular.

Theorem 27. Let $P \in C^{\infty}\left(\mathbb{R}^{n}, \mathbb{R}^{n-1}\right)$ that satisfies Assumptions $\left|\mathcal{A}_{1}\right| \mathcal{A}_{2} \mid \mathcal{A}_{3}$ and $\mid \mathcal{A}_{4}$ then $P$ satisfies Assumption $\mathcal{A}_{5}^{-}$if and only if $\mathrm{Ball}(P)$ is regular in $B_{\mathrm{Ball}}$.

In order to prove Theorem 27, we are going to show that the Jacobian matrices of $\operatorname{Ball}(P)$ and $\operatorname{Ball}(Q)$ evaluated at $X$ have the same rank, where $Q$ is defined in Equation (3.7). Recall that Equation (3.7) implies that $P$ and $Q$ define the same curve around $q$. Notice also that if $X=(q, r, 0) \in \mathbb{R}^{n} \times \mathbb{R}^{n-2} \times \mathbb{R}$ is in $\Omega_{P}^{-1}((q, q))$, then $X \in \Omega_{Q}^{-1}((q, q))$.

Lemma 28. Let $P$ and $Q$ be as defined in 3.7. Under Assumption $\mathcal{A}_{1}$ let $(q, r, 0) \in \mathbb{R}^{n} \times \mathbb{R}^{n-2} \times \mathbb{R}$ be a solution of the system $\mathrm{Ball}(P)$ in $B_{\mathrm{Ball}}$, then $\mathrm{Ball}(P)$ is regular at $(q, r, 0)$ if and only if $\mathrm{Ball}(Q)$ is regular at the point $(0, r, 0) \in \mathbb{R}^{n} \times \mathbb{R}^{n-2} \times \mathbb{R}$ (recall that for simplicity, we assume in Lemma 24 that $q=0 \in \mathbb{R}^{n}$ ).

Proof. Let us write $X=(q, r, 0)$. We are going to prove that the Jacobian matrices of $\operatorname{Ball}(P)$ and $\operatorname{Ball}(Q)$ evaluated at $X$ have the same rank. By Remark 16 we have that $\Omega_{P}(X)=(q, q) \in \widehat{\mathfrak{L}}_{\mathrm{c}}$ (see Definitions 14 and 12 , and hence, $q \in \mathfrak{L}_{\mathrm{c}}$. By Lemma 13 we have that $(0,0, r) \in T_{q} \mathfrak{C}$. We prove the claim in three steps:

Step 1: Let $\widetilde{M}=\left(f_{i j}\right)_{1 \leqslant i, j \leqslant n-1}$ be as defined in the Equality (3.7). We define $S \cdot \widetilde{M}$ (resp. $D \cdot \widetilde{M}$ ) to be the matrix $\left(S \cdot f_{i j}\right)_{1 \leqslant i, j \leqslant n-1}\left(\right.$ resp. $\left.\left(D \cdot f_{i j}\right)_{1 \leqslant i, j \leqslant n-1}\right)$. Using the identity $\frac{1}{2}(a b+c d)=\frac{1}{4}(a+c)(b+d)+\frac{1}{4}(a-$ $c)(b-d)$, one deduces the properties for any $f, g \in C^{\infty}\left(\mathbb{R}^{n}, \mathbb{R}\right)$ :

$$
\begin{aligned}
& S \cdot f g=(S \cdot f)(S \cdot g)+t(D \cdot f)(D \cdot g) \\
& D \cdot f g=(D \cdot f)(S \cdot g)+(S \cdot f)(D \cdot g)
\end{aligned}
$$

These identities applied to Equation (3.7) yield

$$
\left(\begin{array}{c}
S \cdot Q_{1} \\
\cdots \\
\cdots \\
S \cdot Q_{n-1}
\end{array}\right)=\left(\begin{array}{ll}
S \cdot \widetilde{M} & t D \cdot \widetilde{M}
\end{array}\right) \cdot\left(\begin{array}{c}
S \cdot\left(P_{1} \circ \varphi\right) \\
\cdots \\
\cdots \\
S \cdot\left(P_{n-1} \circ \varphi\right) \\
D \cdot\left(P_{1} \circ \varphi\right) \\
\cdots \\
\cdots \\
D \cdot\left(P_{n-1} \circ \varphi\right)
\end{array}\right)
$$


and

$$
\left(\begin{array}{c}
D \cdot Q_{1} \\
\cdots \\
\cdots \\
D \cdot Q_{n-1}
\end{array}\right)=\left(\begin{array}{ll}
D \cdot \widetilde{M} & S \cdot \widetilde{M}
\end{array}\right) \cdot\left(\begin{array}{c}
S \cdot\left(P_{1} \circ \varphi\right) \\
\cdots \\
\cdots \\
S \cdot\left(P_{n-1} \circ \varphi\right) \\
D \cdot\left(P_{1} \circ \varphi\right) \\
\cdots \\
\cdots \\
D \cdot\left(P_{n-1} \circ \varphi\right)
\end{array}\right)
$$

Combining the last two equations:

$$
\left(\begin{array}{c}
S \cdot Q_{1} \\
\cdots \\
\cdots \\
S \cdot Q_{n-1} \\
D \cdot Q_{1} \\
\cdots \\
\cdots \\
D \cdot Q_{n-1}
\end{array}\right)=\left(\begin{array}{cc}
S \cdot \widetilde{M} & t D \cdot \widetilde{M} \\
D \cdot \widetilde{M} & S \cdot \widetilde{M}
\end{array}\right) \cdot\left(\begin{array}{c}
S \cdot\left(P_{1} \circ \varphi\right) \\
\cdots \\
\cdots \\
S \cdot\left(P_{n-1} \circ \varphi\right) \\
D \cdot\left(P_{1} \circ \varphi\right) \\
\cdots \\
\cdots \\
D \cdot\left(P_{n-1} \circ \varphi\right)
\end{array}\right)
$$

${ }_{486} \quad$ Notice that $\left(\begin{array}{cc}S \cdot \widetilde{M} & t D \cdot \widetilde{M} \\ D \cdot \widetilde{M} & S \cdot \widetilde{M}\end{array}\right)_{X}=\left(\begin{array}{cc}\widetilde{M}(q) & 0 \\ D \cdot \widetilde{M}(X) & \widetilde{M}(q)\end{array}\right)$ (recall that in our case we have $S \cdot \widetilde{M}(X)=\widetilde{M}(q)$ ) and that the latter matrix has an inverse (by Lemma $24 \widetilde{M}(q)$ is an invertible matrix of size $n-1$ ), namely, $\left(\begin{array}{cc}\widetilde{M}(q)^{-1} & 0 \\ -\widetilde{M}(q)^{-1} \cdot(D \cdot \widetilde{M})(X) \cdot \widetilde{M}(q)^{-1} & \widetilde{M}(q)^{-1}\end{array}\right)$ which implies (by continuity of the determinant function) 489 that $\left(\begin{array}{cc}S \cdot \widetilde{M} & t D \cdot \widetilde{M} \\ D \cdot \widetilde{M} & S \cdot \widetilde{M}\end{array}\right)$ is invertible in a neighborhood of $X$.

Step 2: Writing $y=\left(y_{3}, \ldots, y_{n}\right)$ and $r=\left(r_{3}, \ldots, r_{n}\right)$, consider the diffeomorphism $\varphi$ defined in Lemma 24 and define the smooth function $\psi$ over an open subset of $\mathbb{R}^{2 n-1}$ containing $X$ which maps the point $\left(x_{1}, x_{2}, y, r, t\right)$ to $\left(\varphi_{1}, \varphi_{2}, S \cdot \varphi_{3}, \ldots, S \cdot \varphi_{n}, D \cdot \varphi_{3}, \ldots, D \cdot \varphi_{n}, t\right)$. Notice that we have:

$$
S \cdot\left(P_{j} \circ \varphi\right)=(S \cdot P) \circ \psi \text { and } D \cdot\left(P_{j} \circ \varphi\right)=(D \cdot P) \circ \psi, \text { for } 1 \leqslant j \leqslant n-1,
$$

since $\varphi_{i}\left(x_{1}, x_{2}, y \pm r \sqrt{t}\right)=\psi_{i} \pm \psi_{n+i-2} \sqrt{\psi_{2 n-1}}$ for all $i \in\{3, \ldots, n\}$. In fact, using the last two equations we can also see that $\psi^{-1}$ exists and is smooth. Thus, $\psi$ is a diffeomorphism.

Step 3: Now, comparing (4.3) with 4.4 we get: 


$$
S D \cdot Q:=\left(\begin{array}{c}
S \cdot Q_{1} \\
\cdots \\
\cdots \\
S \cdot Q_{n-1} \\
D \cdot Q_{1} \\
\cdots \\
\cdots \\
D \cdot Q_{n-1}
\end{array}\right)=\left(\begin{array}{cc}
S \cdot \widetilde{M} & t D \cdot \widetilde{M} \\
D \cdot \widetilde{M} & S \cdot \widetilde{M}
\end{array}\right) \cdot\left(\begin{array}{c}
S \cdot P_{1} \\
\cdots \\
\cdots \\
S \cdot P_{n-1} \\
D \cdot P_{1} \\
\cdots \\
\cdots \\
D \cdot P_{n-1}
\end{array}\right) \circ \psi
$$

Consider the vector $S D \cdot P=\left(S \cdot P_{1}, \ldots, S \cdot P_{n-1}, D \cdot P_{1}, \ldots, D \cdot P_{n-1}\right)^{T}$ and let $J_{S D \cdot P}, J_{S D \cdot Q}$ and $J_{\psi}$ be the Jacobian matrices of $S D \cdot P, S D \cdot Q$ and $\psi$, respectively. Taking the Jacobian matrix of both sides of the last equality:

$$
J_{S D \cdot Q}=\left(\begin{array}{cc}
S \cdot \widetilde{M} & t D \cdot \widetilde{M} \\
D \cdot \widetilde{M} & S \cdot \widetilde{M}
\end{array}\right) \cdot J_{S D \cdot P} \cdot J_{\psi}+\mathrm{Jacobian}\left(\left(\begin{array}{cc}
S \cdot \widetilde{M} & t D \cdot \widetilde{M} \\
D \cdot \widetilde{M} & S \cdot \widetilde{M}
\end{array}\right)\right) \cdot\left(\begin{array}{c}
S \cdot P_{1} \\
\cdots \\
\cdots \\
S \cdot P_{n-1} \\
D \cdot P_{1} \\
\cdots \\
\ldots \\
D \cdot P_{n-1}
\end{array}\right) \circ \psi .
$$

Evaluating the last equality at $X=(0, r, 0)$ and using the fact that $\psi(X)=\psi(0, r, 0)=(0, r, 0)=X$, we note that the second term of the right-hand side is zero. One has:

$$
J_{S D \cdot Q}(X)=\left(\begin{array}{cc}
S \cdot \widetilde{M} & t D \cdot \widetilde{M} \\
D \cdot \widetilde{M} & S \cdot \widetilde{M}
\end{array}\right)_{X} \cdot J_{S D \cdot P}(X) \cdot J_{\psi}(X)
$$

Computing $J_{\psi}(X)$, we get $J_{\psi}(X)=\left(\begin{array}{c:cc}\frac{\partial \varphi_{1}}{\partial z_{1}}(0) & \frac{\partial \varphi_{1}}{\partial z_{2}(0)} & 0_{1 \times(2 n-3)} \\ \hdashline 0_{(2 n-2) \times 1} & I_{2 n-2}\end{array}\right)$, with $\frac{\partial \varphi_{1}}{\partial z_{1}}(0) \neq 0$ according to the formula in Lemma 24

Hence by Equation 4.5, it is straightforward to check that:

$$
\begin{aligned}
J_{\mathrm{Ball}(Q)} & =\left(\begin{array}{c}
J_{S D \cdot Q}(X) \\
2 X
\end{array}\right)=\left(\begin{array}{ccc}
S \cdot \widetilde{M} & t D \cdot \widetilde{M} & 0 \\
D \cdot \widetilde{M} & S \cdot \widetilde{M} & 0 \\
0_{1 \times(n-1)} & 0_{1 \times(n-1)} & 1
\end{array}\right)_{X} \cdot\left(\begin{array}{c}
J_{S D \cdot P}(X) \\
2 X
\end{array}\right) \cdot J_{\psi}(X) \\
& =\left(\begin{array}{ccc}
S \cdot \widetilde{M} & t D \cdot \widetilde{M} & 0 \\
D \cdot \widetilde{M} & S \cdot \widetilde{M} & 0 \\
0_{1 \times(n-1)} & 0_{1 \times(n-1)} & 1
\end{array}\right)_{X} \cdot J_{\mathrm{Ball}(P)}(X) \cdot J_{\psi}(X) .
\end{aligned}
$$



lows

Recalling that $J_{\psi}(X)$ and $\left(\begin{array}{ccc}S \cdot \widetilde{M} & t D \cdot \widetilde{M} & 0 \\ D \cdot \widetilde{M} & S \cdot \widetilde{M} & 0 \\ 0_{1 \times(n-1)} & 0_{1 \times(n-1)} & 1\end{array}\right)_{X}$ are invertible matrices, the proof of the lemma fol-

Now, we are ready to prove Theorem 27, which characterizes the regularity of the solutions of $\operatorname{Ball}(P)$ under generic assumptions. We split the proof in the two Lemmas 32 and 33 Before that, we introduce a new assumption that helps to simplify the proof.

Definition 29. Let $\left(q_{1}, q_{2}\right) \in \widehat{\mathfrak{L}}$. We say that $\left(q_{1}, q_{2}\right)$ satisfies Assumption $\left|\mathcal{A}_{5}^{-1}\right|$ if $q_{1}$ and $q_{2}$ are isolated in $\mathfrak{L}_{\mathrm{n}} \cup \mathfrak{L}_{\mathrm{c}}$ and the following conditions are satisfied:

(a) If $\left(q_{1}, q_{2}\right) \in \widehat{\mathfrak{L}}_{\mathrm{n}}$, then the plane projections of the tangent lines of $q_{1}$ and $q_{2}$ to $\mathfrak{C}$ are linearly independent.

(b) If $\left(q_{1}, q_{2}\right) \in \widehat{\mathfrak{L}}_{\mathrm{c}}$, then the plane projection of a small enough neighborhood of $q_{1}$ in $\mathfrak{C}$ is an ordinary cusp at $\pi_{\mathfrak{C}}\left(q_{1}\right)$ and the multiplicity of the system $\left\{P(x)=0,\left(x_{1}, x_{2}\right)=\pi_{\mathfrak{C}}\left(q_{1}\right)\right\}$ at $q_{1}$ is two.

Assumption $\mathcal{A}_{5}^{-1}$ can be seen as a "local version" of Assumption $\mathcal{A}_{5}^{-}$We are going to prove that if Assumptions $\mathcal{A}_{1}, \mathcal{A}_{2}, \mathcal{A}_{3}$ and $\mathcal{A}_{4}$ are satisfied, then Assumption $\mathcal{A}_{5}^{-}$is equivalent to the condition that Assumption $\mathcal{A}_{5}^{-1}$ is satisfied for all $\widehat{\mathfrak{L}}$.

The main reason behind introducing Assumption $\mathcal{A}_{5}^{-1}$, is that we are going to prove in Lemma 32 that, under Assumption $\mathcal{A}_{1}$ a pair $\left(q_{1}, q_{2}\right) \in \widehat{\mathfrak{L}}$ satisfies Assumption $\mathcal{A}_{5}^{-1}$ if and only if every $X$ in $\Omega_{P}^{-1}\left(q_{1}, q_{2}\right)$ is a regular solution of $\operatorname{Ball}(P)$, whereas Assumption $\mathcal{A}_{5}^{-}$is, in general, not sufficient for the regularity of the solutions of Ball $(P)$. For example, take $n=3$ and $P=\left(x_{1}-x_{3}^{6}, x_{2}-x_{3}^{9}\right)$. We can see that $P$ satisfies Assumption $\mathcal{A}_{1}$ the set $\mathfrak{L}_{\mathrm{c}}$ consists of a unique point $q=(0,0,0)$ and the set $\mathfrak{L}_{\mathrm{n}}$ is empty. The plane projection of $\mathfrak{C}$ is the curve given by the equation $x_{1}^{3}-x_{2}^{2}=0$. Hence, Assumption $\mathcal{A}_{5}^{-}$is satisfied. However, the multiplicity of the system $\left\{P\left(x_{1}, x_{2}, x_{3}\right)=0 \in \mathbb{R}^{2}, x_{1}=x_{2}=0\right\}$ at the point $q$ equals 6 (Lemma 23). Hence, Assumption $\mathcal{A}_{5}^{-1}$ is not satisfied and one can also check that $\operatorname{Ball}(P)$ is not regular.

The next definition and lemma are technical tools to handle the case of nodes in Lemma 32, and later in Lemma 54

Definition 30. Consider $P=\left(P_{1}, \ldots, P_{n-1}\right) \in C^{\infty}\left(\mathbb{R}^{n}, \mathbb{R}^{n-1}\right)$ satisfying Assumption $\mathcal{A}_{1}$ and recall that we denote the Jacobian matrix of $P$ at the point $q$ by $J_{P}(q)$. We define the $(n-1) \times(n-2)$ sub-matrix $M_{P}(q)$ obtained by removing the first two columns of $J_{P}(q)$ and the $(n-1) \times 2$ sub-matrix $N_{P}(q)$ formed by the first two columns of $J_{P}(q)$. Let $q_{1}, q_{2} \in \mathfrak{C}$, we define the $2 n-2$ square matrix $M\left(q_{1}, q_{2}\right)=\left(\begin{array}{ccc}N_{P}\left(q_{1}\right) & 0 & M_{P}\left(q_{1}\right) \\ N_{P}\left(q_{2}\right) & M_{P}\left(q_{2}\right) & 0\end{array}\right)$.

Lemma 31. Using the same assumption and notation as in Definition 30 let $q_{1}$ and $q_{2}$ be distinct points of $\mathfrak{C}$ with $\pi_{\mathfrak{C}}\left(q_{1}\right)=\pi_{\mathfrak{C}}\left(q_{2}\right)$, then $M\left(q_{1}, q_{2}\right)$ is invertible if and only if neither $q_{1}$ nor $q_{2}$ is in $\mathfrak{L}_{\mathfrak{c}}$ and the plane projections of the tangent lines of $\mathfrak{C}$ at $q_{1}$ and $q_{2}$ do not coincide. 
Proof. We prove the converse statement using

$\operatorname{det}\left(M\left(q_{1}, q_{2}\right)\right)=0 \Longleftrightarrow \quad$ There exist $\alpha \in \mathbb{R}^{2}$ and $\beta, \gamma \in \mathbb{R}^{n-2}$ such that the vector $x=(\alpha, \beta, \gamma)$ is not trivial and $M\left(q_{1}, q_{2}\right) \cdot x^{T}=0$.

$\Longleftrightarrow \quad(\alpha, \beta)$ and $(\alpha, \gamma)$ are in the tangent lines $T_{q_{1}} \mathfrak{C}$ and $T_{q_{2}} \mathfrak{C}$, respectively, and at least one of them is not trivial.

The last statement can be split in two cases:

- $\alpha$ is not trivial, which is equivalent to saying that the plane projections of $T_{q_{1}} \mathfrak{C}$ and $T_{q_{2}} \mathfrak{C}$ are both generated by $\alpha$ and coincide.

- $\alpha=(0,0)$, which is equivalent to $\beta$ or $\gamma$ are not trivial, which is equivalent to $T_{q_{2}} \mathfrak{C}$ or $T_{q_{1}} \mathfrak{C}$ projects to a point in the plane, which is equivalent to $q_{1}$ or $q_{2}$ is in $\mathfrak{L}_{\mathrm{c}}$.

Lemma 32. Let $P \in C^{\infty}\left(\mathbb{R}^{n}, \mathbb{R}^{n-1}\right)$ satisfy Assumption $\mathcal{A}_{1}$ Let $X$ be a solution of $\operatorname{Ball}(P)$ and $\left(q_{1}, q_{2}\right)=$ $\Omega_{P}(X)$ (Definition 14 ), then $X$ is a regular solution of $\operatorname{Ball}(P)$ if and only if $\left(q_{1}, q_{2}\right)$ satisfies Assumption $\mathcal{A}_{5}^{-\prime}$

Proof. Let $X=\left(x_{1}, x_{2}, y, r, t\right) \in \mathbb{R} \times \mathbb{R} \times \mathbb{R}^{n-2} \times \mathbb{R}^{n-2} \times \mathbb{R}$ be a solution of Ball $(P)$. We consider two cases:

Case (a): $t \neq 0$, i.e., $q_{1} \neq q_{2}$.

It is easy to see that $\frac{\partial\left(S \cdot P_{i}\right)}{\partial x_{j}}, \frac{\partial\left(D \cdot P_{i}\right)}{\partial x_{j}}, \frac{\partial\left(S \cdot P_{i}\right)}{\partial r_{k}}, \frac{\partial\left(D \cdot P_{i}\right)}{\partial r_{k}}, \frac{\partial\left(S \cdot P_{i}\right)}{\partial t}, \frac{\partial\left(D \cdot P_{i}\right)}{\partial t}$ are, respectively, equal to: $S \cdot \frac{\partial\left(P_{i}\right)}{\partial x_{j}}, D$. $\frac{\partial\left(P_{i}\right)}{\partial x_{j}}, t \cdot D \cdot \frac{\partial\left(P_{i}\right)}{\partial x_{k}}, S \cdot \frac{\partial\left(P_{i}\right)}{\partial x_{k}}, \frac{1}{2} \sum_{m=3}^{n} D \cdot\left(\frac{\partial P_{i}}{\partial x_{m}}\right) \cdot r_{m}, \frac{1}{2 t}\left[\sum_{m=3}^{n} S \cdot\left(\frac{\partial P_{i}}{\partial x_{m}}\right) \cdot r_{m}-D \cdot P_{i}\right]$. Hence, by computing the Jacobian matrix of the $\operatorname{Ball}(P)$ we get the matrix:

$$
\left(\begin{array}{cccccc}
S \cdot \frac{\partial\left(P_{1}\right)}{\partial x_{1}} & \ldots & S \cdot \frac{\partial P_{1}}{\partial x_{n}} & t \cdot D \cdot \frac{\partial\left(P_{1}\right)}{\partial x_{3}} \ldots & t \cdot D \cdot \frac{\partial\left(P_{1}\right)}{\partial x_{n}} & \frac{1}{2} \sum_{m=3}^{n} D \cdot\left(\frac{\partial P_{1}}{\partial x_{m}}\right) \cdot r_{m} \\
\ldots & \ldots & \ldots & \ldots & \ldots \\
\ldots & \ldots & \ldots & \ldots & \ldots \\
S \cdot \frac{\partial\left(P_{n-1}\right)}{\partial x_{1}} & \ldots & S \cdot \frac{\partial\left(P_{n-1}\right)}{\partial x_{n}} & t \cdot D \cdot \frac{\partial\left(P_{n-1}\right)}{\partial x_{3}} \ldots & t \cdot D \cdot \frac{\partial\left(P_{n-1}\right)}{\partial x_{n}} & \frac{1}{2} \sum_{m=3}^{n} D \cdot\left(\frac{\partial P_{n-1}}{\partial x_{m}}\right) \cdot r_{m} \\
D \cdot \frac{\partial\left(P_{1}\right)}{\partial x_{1}} & \ldots & D \cdot \frac{\partial\left(P_{1}\right)}{\partial x_{n}} & S \cdot \frac{\partial\left(P_{1}\right)}{\partial x_{3}} \ldots & S \cdot \frac{\partial\left(P_{1}\right)}{\partial x_{n}} & \frac{1}{2 t}\left[\sum_{m=3}^{n} S \cdot\left(\frac{\partial P_{1}}{\partial x_{m}}\right) \cdot r_{m}-D \cdot P_{1}\right] \\
\ldots & \ldots & \ldots & \ldots & \ldots \\
\ldots & \ldots & \ldots & \ldots & \ldots \cdot \frac{\partial\left(P_{n-1}\right)}{\partial x_{n}} & \frac{1}{2 t}\left[\sum_{m=3}^{n} S \cdot\left(\frac{\partial P_{n-1}}{\partial x_{m}}\right) \cdot r_{m}-D \cdot P_{n-1}\right] \\
D \cdot \frac{\partial\left(P_{n-1}\right)}{\partial x_{1}} & \ldots & D \cdot \frac{\partial\left(P_{n-1}\right)}{\partial x_{n}} & S \cdot \frac{\partial\left(P_{n-1}\right)}{\partial x_{3}} \ldots & 2 r_{n} & 0 \\
0 & \ldots & 0 & 2 r_{3} \ldots &
\end{array}\right) .
$$

We denote by $C_{i}$ (resp. $L_{i}$ ) the $i$-th column (resp. line) of the latter matrix. Replace the last column $C_{2 n-1}$ with $\sum_{m=1}^{n-2} \frac{r_{m+2}}{2 t} C_{n+m}+C_{2 n-1}$, also for all integers $1 \leqslant k \leqslant n-1$ we replace the line $L_{k}$ with $L_{k}+\sqrt{t} \cdot L_{k+n-1}$ and 
then the line $L_{k+n-1}$ with $L_{k}-2 \sqrt{t} L_{k+n-1}$. The resulting matrix is:

$$
\left(\begin{array}{cccccc}
\frac{\partial\left(P_{1}\right)}{\partial x_{1}}\left(q_{1}\right) & \ldots \frac{\partial P_{1}}{\partial x_{n}}\left(q_{1}\right) & \sqrt{(t)} \cdot \frac{\partial\left(P_{1}\right)}{\partial x_{3}}\left(q_{1}\right) & \ldots & \sqrt{(} t) \frac{\partial\left(P_{1}\right)}{\partial x_{n}}\left(q_{1}\right) & 0 \\
\ldots & \ldots & \ldots & \ldots & \ldots \\
\frac{\partial\left(P_{n-1}\right)}{\partial x_{1}}\left(q_{1}\right) & \ldots \frac{\partial P_{n-1}}{\partial x_{n}}\left(q_{1}\right) & \sqrt{(t) \cdot \frac{\partial\left(P_{n-1}\right)}{\partial x_{3}}\left(q_{1}\right)} & \ldots & \sqrt{\left.(t) \frac{\partial\left(P_{n-1}\right)}{\partial x_{n}}\left(q_{1}\right)\right)} & 0 \\
\frac{\partial\left(P_{1}\right)}{\partial x_{1}}\left(q_{2}\right) & \ldots \frac{\partial\left(P_{1}\right)}{\partial x_{n}}\left(q_{2}\right) & -\sqrt{(} t) \frac{\partial\left(P_{1}\right)}{\partial x_{3}}\left(q_{2}\right) & \ldots & -\sqrt{(t)} \frac{\partial\left(P_{1}\right)}{\partial x_{n}}\left(q_{2}\right) & 0 \\
\ldots & \ldots & \ldots & \ldots & \ldots & \\
\ldots & \ldots & \ldots & \ldots & \ldots & \ldots \\
\frac{\partial\left(P_{n-1}\right)}{\partial x_{1}}\left(q_{2}\right) & \ldots \frac{\partial\left(P_{n-1}\right)}{\partial x_{n}}\left(q_{2}\right) & -\sqrt{(} t) \frac{\partial\left(P_{n-1}\right)}{\partial x_{3}}\left(q_{2}\right) & \ldots & \left.-\sqrt{(} t) \frac{\partial\left(P_{n-1}\right)}{\partial x_{n}}\left(q_{2}\right)\right) & 0 \\
0 \ldots & 0 & 2 r_{3} & \ldots & 2 r_{n} & \frac{1}{2 t}
\end{array}\right) .
$$

The determinant of the latter matrix is zero if and only if the determinant of the following matrix is zero:

$$
M_{0}=\left(\begin{array}{lll}
N_{P}\left(q_{1}\right) & M_{P}\left(q_{1}\right) & M_{P}\left(q_{1}\right) \\
N_{P}\left(q_{2}\right) & M_{P}\left(q_{2}\right) & -M_{P}\left(q_{2}\right)
\end{array}\right) \text {, where } M_{P}\left(q_{1}\right), M_{P}\left(q_{2}\right) \text { are the minors that are obtained, }
$$
respectively, by removing the first two columns from $J_{P}\left(q_{1}\right), J_{P}\left(q_{2}\right)$ and $N_{P}\left(q_{1}\right), N_{P}\left(q_{2}\right)$ are the matrices formed by the first two columns of $J_{P}\left(q_{1}\right), J_{P}\left(q_{2}\right)$, respectively. By linear operations on $M_{0}$, we can see that $M_{0}$ has same rank as the matrix $M\left(q_{1}, q_{2}\right)$ (see Definition 30). Thus, $X$ is regular for Ball $(P)$ if and only if $M\left(q_{1}, q_{2}\right)$ is invertible. By Lemma 31 we have that $M\left(q_{1}, q_{2}\right)$ is invertible if and only if none of $q_{1}, q_{2}$ is in $\mathfrak{L}_{\mathrm{c}}$ (and hence none of the plane projections of $T_{q_{1}} \mathfrak{C}, T_{q_{2}} \mathfrak{C}$ is trivial) and the plane projection of their tangent spaces are different. Equivalently, the pair $\left(q_{1}, q_{2}\right)$ is in $\widehat{\mathfrak{L}}_{\mathrm{n}}$ and satisfies Assumption $\mathcal{A}_{5}^{-1}$

Case (b): $t=0$, i.e., $q_{1}=q_{2}$.

Let us write $q=q_{1}$. We prove the claim in three steps:

Step 1: We first simplify $P$. Without loss of generality and by Lemma 22 we can assume that $q=0$ and $P_{1}, \ldots, P_{n-1}$ are, respectively, equal to $x_{1}-f_{1}\left(x_{n}\right), x_{2}-f_{2}\left(x_{n}\right), \ldots, x_{n-1}-f_{n-1}\left(x_{n}\right)$ with the property that $\min \left\{\operatorname{mult}\left(f_{1}\right), \operatorname{mult}\left(f_{2}\right)\right\} \geqslant 2$. For all $i \in\{3, \ldots, n-1\}$, using Taylor's theorem, we can write $f_{i}\left(x_{n}\right)=$ $\sum_{j=1}^{3} a_{i, j} x_{n}^{j}+x_{n}^{4} h_{i}\left(x_{n}\right)$, for some $a_{i, j} \in \mathbb{R}$ and smooth functions $h_{i}\left(x_{n}\right)$. Since $\min \left\{\operatorname{mult}\left(f_{1}\right), \operatorname{mult}\left(f_{2}\right)\right\} \geqslant 2$, we can write $f_{1}\left(x_{n}\right)=\sum_{j=2}^{3} \alpha_{j} x_{n}^{j}+x_{n}^{4} h_{1}\left(x_{n}\right)$ and $f_{2}\left(x_{n}\right)=\sum_{j=2}^{3} \beta_{j} x_{n}^{j}+x_{n}^{4} h_{2}\left(x_{n}\right)$. Notice that

$$
\left(f_{1}\left(x_{n}\right), f_{2}\left(x_{n}\right), f_{3}\left(x_{n}\right), \ldots, f_{n-1}\left(x_{n}\right), x_{n}\right)
$$

is a local parameterization system of $\mathfrak{C}$ around $q$. Since $\operatorname{dim}\left(T_{q} \mathfrak{C}\right)=1$ (Assumption $\left[\mathcal{A}_{1}\right.$, there exists $\lambda \in \mathbb{R}^{*}$ with $\left(a_{3,1}, \ldots, a_{n-1,1}, 1\right)=\lambda r$ (because the vectors $(0,0, r) \in \mathbb{R} \times \mathbb{R} \times \mathbb{R}^{n-2}$ and $\left(0,0, a_{1,3}, \ldots, a_{1, n-1}, 1\right)$ are in $\left.T_{q} \mathfrak{C} \backslash\{0\}\right)$. In particular, $r_{n} \neq 0$.

Step 2: Now, we compute $J_{\mathrm{Ball}(P)}$ at $X=\left(x_{1}, x_{2}, y, r, 0\right)$ by first computing it for $X_{t}=\left(x_{1}, x_{2}, y, r, t\right)$ with $t \neq 0$, and then taking the limit when $t$ goes to 0 . Since the operator $S$ is linear, we write $S\left(x_{i}-f_{i}\left(x_{n}\right)\right)=$ $S\left(x_{i}-\sum_{j=1}^{3} a_{i, j} x_{n}^{j}\right)-S\left(x_{n}^{4} h_{i}\left(x_{n}\right)\right)$. On the other hand, using the identity 4.1) we deduce that $S\left(x_{n}^{4} h_{i}\left(x_{n}\right)\right)=$ 
$S\left(x_{n}^{4}\right) \cdot S\left(h_{i}\left(x_{n}\right)\right)+t D\left(x_{n}^{4}\right) \cdot D\left(h_{i}\left(x_{n}\right)\right)$, for all $\in\{1, \ldots, n-1\}$. It is straightforward to see that $S\left(x_{n}^{4}\right)=$ $r_{n}^{4} t^{2}+6 r_{n}^{2} t x_{n}^{2}+x_{n}^{4}$ and $t D\left(x_{n}^{4}\right)=4 r_{n}^{3} x_{n} t^{2}+4 r_{n} x_{n}^{3} t$ with $r=\left(r_{3}, \ldots, r_{n}\right)$. Hence, all of the first-order partial derivatives of $S\left(x_{n}^{4} h_{i}\left(x_{n}\right)\right)$, evaluated at $X_{t}$, converge to zero when $t$ goes to 0 . Hence, the partial derivatives of the functions $S\left(x_{i}-f_{i}\left(x_{n}\right)\right)$ and $S\left(x_{i}-\sum_{j=1}^{3} a_{i, j} x_{n}^{j}\right)$ evaluated at $X$ are equal. Using an analogous argument, we deduce that the evaluation of the partial derivatives of the functions $D\left(x_{i}-f_{i}\left(x_{n}\right)\right)$ and $D\left(x_{i}-\sum_{j=1}^{3} a_{i, j} x_{n}^{j}\right)$, at $X$ are also equal. Thus, $J_{\mathrm{Ball}(P)}\left(X_{t}\right)$ and $J_{\mathrm{Ball}(\bar{P})}\left(X_{t}\right)$ converge to the same limit $J_{\mathrm{Ball}(P)}(X)$, where $\bar{P}$ is the function obtained by truncating $P$ beyond degree 3 with respect to the variable $x_{n}$.

Computing $J_{\operatorname{Ball}(P)}(X)=\lim _{t \rightarrow 0} J_{\operatorname{Ball}(\bar{P})}\left(X_{t}\right)$, we get:

$$
\left(\begin{array}{ccccccccc}
1 & 0 & \ldots & 0 & 0 & 0 & \ldots & 0 & -\alpha_{2} r_{n}^{2} \\
0 & 1 & \ldots & 0 & 0 & 0 & \ldots & 0 & -\beta_{2} r_{n}^{2} \\
0 & 0 & \ldots & \ldots & -a_{3,1} & 0 & \ldots & 0 & -a_{3,2} r_{n}^{2} \\
& \ldots & \ldots & \ldots & \ldots & \ldots & \ldots & & \\
& \ldots & \ldots & \ldots & \ldots & \ldots & \ldots & & \\
0 & 0 & \ldots & 1 & -a_{n-1,1} & 0 & \ldots & 0 & -a_{n-1,2} r_{n}^{2} \\
0 & 0 & \ldots & \ldots & -2 \alpha_{2} r_{n} & 0 & \ldots & 0 & -\alpha_{3} r_{n}^{3} \\
0 & 0 & \ldots & \ldots & -2 \beta_{2} r_{n} & 0 & \ldots & 0 & -\beta_{3} r_{n}^{3} \\
0 & 0 & \ldots & \ldots & -2 a_{3,2} r_{n} & 1 & \ldots & -a_{3,1} & -a_{3,3} r_{n}^{3} \\
& \ldots & \ldots & \ldots & \ldots & \ldots & \ldots & & \\
& \ldots & \ldots & \ldots & \ldots & \ldots & \ldots & & \\
0 & 0 & \ldots & \ldots & -2 a_{n-1,2} r_{n} & 0 & \ldots 1 & -a_{n-1,1} & -a_{n-1,3} r_{n}^{3} \\
0 & 0 & \ldots & \ldots & 0 & 2 r_{3} & \ldots 2 r_{n-1} & 2 r_{n} & 0
\end{array}\right)
$$

Hence, observing that the matrix is block diagonal, its determinant is zero if and only if the determinant of the following one is:

$$
\left(\begin{array}{cccccc}
-2 \alpha_{2} r_{n} & 0 & \ldots & 0 & 0 & -\alpha_{3} r_{n}^{3} \\
-2 \beta_{2} r_{n} & 0 & \ldots & 0 & 0 & -\beta_{3} r_{n}^{3} \\
-2 a_{3,2} r_{n} & 1 & 0 \ldots & 0 & -a_{3,1} & -a_{3,3} r_{n}^{3} \\
& \ldots & \ldots & & & \\
-2 a_{n-1,2} r_{n} & 0 & 0 \ldots & 1 & -a_{n-1,1} & -a_{n-1,3} r_{n}^{3} \\
0 & 2 r_{3} & \ldots & 2 r_{n-1} & 2 r_{n} & 0
\end{array}\right)
$$


Shifting the columns of the last matrix we get:

$$
\left(\begin{array}{cccccc}
-\alpha_{3} r_{n}^{3} & -2 \alpha_{2} r_{n} & 0 & \ldots & 0 & 0 \\
-\beta_{3} r_{n}^{3} & -2 \beta_{2} r_{n} & 0 & \ldots & 0 & 0 \\
-a_{3,3} r_{n}^{3} & -2 a_{3,2} r_{n} & 1 & 0 \ldots & 0 & -a_{3,1} \\
& \ldots & \ldots & & & \\
-a_{n-1,3} r_{n}^{3} & -2 a_{n-1,2} r_{n} & 0 & 0 \ldots & 1 & -a_{n-1,1} \\
0 & 0 & 2 r_{3} & \ldots & 2 r_{n-1} & 2 r_{n}
\end{array}\right)
$$

To compute the determinant of the second block, we expand it about the last row. Hence, the determinant of the last matrix is zero if and only if $r_{n}\left(\alpha_{2} \beta_{3}-\alpha_{3} \beta_{2}\right)\left(r_{n}+\sum_{i=3}^{n-1} a_{i, 1} r_{i}\right)=0$. Notice that, by Step 1 , we have that $r_{n} \neq 0$ and the third factor $\left(r_{n}+\sum_{i=3}^{n-1} a_{i, 1} r_{i}\right)$ is never zero since it is equal to $\lambda$. Thus, $J_{\operatorname{Ball}(P)}(X)$ is invertible iff $\alpha_{2} \beta_{3}-\alpha_{3} \beta_{2} \neq 0$, equivalently, the matrix $A=\left(\begin{array}{cc}\alpha_{2} & \alpha_{3} \\ \beta_{2} & \beta_{3}\end{array}\right)$ is invertible.

Step 3: We now show that the invertibility of $A$ is equivalent to the condition that $(q, q)$ satisfies Assumption $\mathcal{A}_{5}^{-1}$

First assume that $A$ is invertible. It follows that either $\alpha_{2} \neq 0$ or $\beta_{2} \neq 0$ and this yields that the minimum of the multiplicities of $f_{1}$ and $f_{2}$ is 2 . By Lemma 23 , the multiplicity of the system $\left\{P\left(x_{1}, x_{2}, y\right)=0 \in\right.$ $\left.\mathbb{R}^{n-1},\left(x_{1}, x_{2}\right)=\pi_{\mathfrak{C}}(q)\right\}$ at $q$ is equal to 2, thus Assumption $\mathcal{A}_{5}^{-1}$ (b) is satisfied. Using the same notation as in the proof of Lemma 24 , one can write $\tilde{f}_{1}\left(z_{n}\right)=z_{n}^{2}\left(\xi_{1}\left(z_{n}^{2}\right)+z_{n} \xi_{2}\left(z_{n}^{2}\right)\right)$. Notice that $\xi_{2}\left(x_{n}^{2}\right)$ cannot be the zero function, otherwise $\tilde{f}_{1}(\epsilon)=\tilde{f}_{1}(-\epsilon)$ and $\tilde{f}_{2}(\epsilon)=\tilde{f}_{2}(-\epsilon)$ for all small enough $\epsilon>0$, which means that $X$ would be the limit of solutions $X_{\epsilon}$ of $\operatorname{Ball}(P)$ with $\Omega_{P}\left(X_{\epsilon}\right) \in \widehat{\mathfrak{L}}_{\mathrm{n}} . X$ would then be a non-isolated solution and thus a non-regular solution of Ball $(P)$ which contradicts the assumption. We then have two cases as in Lemma 24. The first one is when $\operatorname{mult}\left(\xi_{2}\left(z_{n}\right)\right)=\infty$, that would imply that $\alpha_{2}=\alpha_{3}=0$ and contradicts the invertibility of $A$. We then must satisfy the second case $\operatorname{mult}\left(\xi_{2}\left(z_{n}\right)\right)=k<\infty$ and, after a change of variables, the first equation of the system becomes equivalent to $z_{1}-z_{n}^{2 k+3}=0$. The invertibility of $A$ implies that $k=0$. The projection of the curve in the plane is thus locally parameterized by $\left(z_{n}^{3}, z_{n}^{2}\right)$ and is an ordinary cusp, Assumption $\mathcal{A}_{5}^{-1}$ (a) is satisfied.

Second, assume that Assumption $\mathcal{A}_{5}^{-1}$ is satisfied. By Lemma 23 and Assumption $\mathcal{A}_{5}^{-1}$ (b), the minimum of the multiplicities of $f_{1}$ and $f_{2}$ is 2 . Using the proof of Lemma 24 once again, one can assume that $f_{2}\left(z_{n}\right)=z_{n}^{2}$ and $f_{1}\left(z_{n}\right)=z_{n} g\left(z_{n}^{2}\right)$ or $f_{1}\left(z_{n}\right)=z_{n}^{2 k+3}$. By Assumption $\mathcal{A}_{5}^{-1}$ (a), the projection is an ordinary cusp and thus has a parameterization of the form $\left(z_{n}^{2}, z_{n}^{3}\right)$, that is $f_{1}\left(z_{n}\right)=z_{n}^{3}$. This implies that $A$ is equivalent to $\left(\begin{array}{ll}0 & 1 \\ 1 & 0\end{array}\right)$ and hence is invertible.

Lemma 33. If Assumptions $\mathcal{A}_{1}, \mathcal{A}_{2}, \mathcal{A}_{3}$ and $\mathcal{A}_{4}$ are satisfied, then Assumption $\mathcal{A}_{5}^{-}$is satisfied if and only if Assumption $\mathcal{A}_{5}^{-1}$ is satisfied for all $\left(q_{1}, q_{2}\right) \in \widehat{\mathfrak{L}} \subset B \times B$. 
Proof. Assume that Assumption $\overline{\mathcal{A}_{5}^{-}}$is satisfied and $\left(q_{1}, q_{2}\right) \in \widehat{\mathfrak{L}}$. If $\left(q_{1}, q_{2}\right) \in \widehat{\mathfrak{L}}_{\mathrm{c}}$, then by Lemma 24 and Assumption $\mathcal{A}_{5}^{-}$we must have that the plane projection of a small enough neighborhood of $q_{1}$ in $\mathfrak{C}$ is an ordinary cusp at $\pi_{\mathfrak{C}}\left(q_{1}\right)$. By Assumption $\mathcal{A}_{3}$ and Lemma 20, the multiplicity of the mentioned system at $q_{1}=q_{2}$ is two. Thus, $\left(q_{1}, q_{2}\right)$ satisfies Assumption $\mathcal{A}_{5}^{-1}$ If $\left(q_{1}, q_{2}\right) \in \widehat{\mathfrak{L}}_{\mathrm{n}}$, then by Lemma 21 and Assumption $\mathcal{A}_{5}^{-}$, we have that $\pi_{\mathfrak{C}}\left(q_{1}\right)$ is a node in $\pi_{\mathfrak{C}}(\mathfrak{C})$. Thus, we have that $\pi_{\mathfrak{C}}\left(q_{1}\right)$ is a transverse intersection of two smooth branches of $\pi_{\mathfrak{C}}(\mathfrak{C})$. Those branches are the plane projections of two disjoint branches of $\mathfrak{C}$ each of which contains either $q_{1}$ or $q_{2}$. Hence, the plane projections of the tangent spaces of $q_{1}$ and $q_{2}$ to $\mathfrak{C}$ are linearly independent. Thus, $\left(q_{1}, q_{2}\right)$ satisfies Assumption $\mathcal{A}_{5}^{-1}$

Assume conversely that $\mathcal{A}_{5}^{-1}$ is satisfied for all $\left(q_{1}, q_{2}\right) \in \widehat{\mathfrak{L}}$. By Lemma 26 , any singular point of $\pi_{\mathfrak{C}}(\mathfrak{C})$ is the plane projection of a point $q_{1} \in \mathfrak{L}_{\mathrm{c}} \cup \mathfrak{L}_{\mathrm{n}}$. For some $q_{2} \in \mathfrak{C}$, the pair $\left(q_{1}, q_{2}\right)$ is in $\widehat{\mathfrak{L}}$ (which satisfies Assumption $\mathcal{A}_{5}^{-1}$. Hence, if $\left(q_{1}, q_{2}\right)$ is in $\widehat{\mathfrak{L}}_{\mathrm{n}}$ (resp. in $\widehat{\mathfrak{L}}_{\mathrm{c}}$ ) the plane projection of $q_{1}$ is a node (resp. an ordinary cusp) by Lemma21 (resp. Lemma24).

\section{Lemmas 32 and 33 then imply Theorem 27}

\section{Semi-algorithms to check assumptions and isolate singularities}

In this section we present Semi-algorithm 3 that checks the weak assumptions of Section 2.4 and, if it terminates, outputs a superset of isolating boxes of the singularities of the plane projection $\pi_{\mathfrak{C}}(\mathfrak{C})$ of $\mathfrak{C}$. We also present Semi-algorithm 4 that checks the strong assumptions of Section 2.4 and, if it terminates, outputs a set of isolating boxes of the singularities of $\pi_{\mathfrak{C}}(\mathfrak{C})$.

The main idea of these semi-algorithms comes from Theorems 11 and 27. Theorem 11 states that, under Assumptions $\mathcal{A}_{1-4}$, the singularities of $\pi_{\mathfrak{C}}(\mathfrak{C})$ are the plane projections of the solutions of $\operatorname{Ball}(P)$. Theorem 27 states that, under the further Assumption $\mathcal{A}_{5}^{-}$Ball $(P)$ is regular, so we can use certified numerical methods such as interval Newton methods [MKC09] to solve $\operatorname{Ball}(P)$. In addtion, in order to verify these assumptions, we use subdivision approaches based on interval arithmetic in a semi-algorithm framework.

We present in Section 5.1 the basics of interval arithmetic with the notation and definitions by Lin and Yap [LY11] and our semi-algorithms in Section 5.2.

\subsection{Interval arithmetic}

Recall that for some positive integer $k$, by a closed (resp. open) $k$-box $\mathfrak{B}$, we mean the Cartesian product of $k$ closed (resp. open) intervals. The width of a box $\mathfrak{B}$, denoted by $w(\mathfrak{B})$, is the maximal length of the intervals of that product. For a subset $A \subset \mathbb{R}^{k}$, the set $I A$ is the set of all closed $k$-boxes that are contained in $A$. For the positive integer $m$ and a function $f: A \rightarrow \mathbb{R}^{m}$, the function $\square f: I A \rightarrow I \mathbb{R}^{m}$ is called an inclusion of $f$ if the set $f(\mathfrak{B})=\{f(x) \mid x \in \mathfrak{B}\}$ is contained in $\square f(\mathfrak{B})$, for all $\mathfrak{B} \in I A$. An inclusion $\square f$ of $f$ is called a box function, if for any descending sequence of closed $k$-boxes $\mathfrak{B}_{1} \supset \mathfrak{B}_{2} \supset \ldots$ that converges to a point $q \in \mathbb{R}^{k}$, the sequence $\square f\left(\mathfrak{B}_{1}\right) \supset \square f\left(\mathfrak{B}_{2}\right) \supset \ldots$ converges to $f(q)$. In the rest of this section, we assume that we are given a 
box function $\square f$ for any function $f$ we consider. The command subdivide is applied to a closed $k$-box $\overline{\mathfrak{B}}$, and it returns the set of boxes obtained by bisecting $\overline{\mathfrak{B}}$ in all dimensions.

An interval matrix $\square M$ is a matrix whose coefficients are intervals. It can also be seen as the set of all matrices whose $(i, j)$-th coefficients belong to the $(i, j)$-th interval. The rank of an interval matrix $\square M$, denoted by $\operatorname{rank}(\square M)$, is the minimum of the ranks of all the matrices in this set.

\subsection{Semi-algorithms}

This section is dedicated to the proof of the following theorem. Recall that the weak and strong assumptions are defined in Definition 8

Theorem 34. For an open $n$-box $B$ and a smooth function $P$ from $\bar{B}$ to $\mathbb{R}^{n-1}$, Semi-algorithm 3 stops if and only if $P$ satisfies the weak assumptions in $\bar{B}$ and then it returns a set of isolating boxes of all the singularities of $\pi_{\mathfrak{C}}(\mathfrak{C})$, plus possibly other spurious disjoint boxes. Semi-algorithm 4 stops if and only if $P$ satisfies the strong assumptions in $\bar{B}$ and then it returns a set of isolating boxes of all the singularities of $\pi_{\mathfrak{C}}(\mathfrak{C})$.

To check whether a given function $P$ satisfies the weak assumptions $\mathcal{A}_{1}, \mathcal{A}_{2}, \mathcal{A}_{3}, \mathcal{A}_{4}$ and $\mathcal{A}_{5}^{-}$in $\bar{B}$, we use their relation to the solutions of $\operatorname{Ball}(P)$ studied in the previous sections. Recall that for any subset $A \subseteq \mathbb{R}^{n}$, we defined $A_{\text {Ball }}=\left\{\left(x_{1}, x_{2}, y, r, t\right) \mid t \geqslant 0,\left(x_{1}, x_{2}, y+r \sqrt{t}\right),\left(x_{1}, x_{2}, y-r \sqrt{t}\right) \in A,\|r\|^{2}=1\right\}$. Let $B$ be an open $n$-box and $P$ be a smooth function from $\bar{B}$ to $\mathbb{R}^{n-1}$ that satisfies Assumption $\mathcal{A}_{1}$ in $\bar{B}$. Consider the following assumptions:

$\aleph_{1}$ - All solutions of $\operatorname{Ball}(P)$ in $\bar{B}_{\text {Ball }}$ are regular.

$\aleph_{2}$ - For every solution $X$ of $\operatorname{Ball}(P)$ in $\bar{B}_{\text {Ball }}$, none of the points of the pair $\Omega_{P}(X)$ (Definition 14 is in the boundary of $B$.

$\aleph_{3}$ - No two distinct solutions of $\operatorname{Ball}(P)$ in $\bar{B}_{\text {Ball }}$, except the twin solutions (Remark 17), have the same plane projection.

The next lemma shows the relation between these new assumptions and those of Section 2.4 The motivation of these alternative assumptions is that they are stated in terms of Ball $(P)$, which makes them easier to verify in our semi-algorithms.

Lemma 35. Let $B$ be an open $n$-box and $P$ be a smooth function from $\bar{B}$ to $\mathbb{R}^{n-1}$ that satisfies Assumption $\mathcal{A}_{1}$ in $\bar{B}$. Then, Assumptions $\aleph_{1}, \aleph_{2}$ and $\aleph_{3}$ are satisfied if and only if Assumptions $\mathcal{A}_{2}, \mathcal{A}_{3}, \mathcal{A}_{4}$ and $\mathcal{A}_{5}^{-}$are satisfied in $\bar{B}$.

Proof. If Assumptions $\mathcal{A}_{2}, \mathcal{A}_{3}, \mathcal{A}_{4}$ and $\mathcal{A}_{5}^{-}$are satisfied in $\bar{B}$, then by Theorem 27 we have Assumption $\aleph_{1}$ is satisfied. Moreover, by Assumptions $\mathcal{A}_{2}$ and $\mathcal{A}_{4}$ we have that none of $\overline{\mathfrak{L}_{\mathrm{n}}}, \overline{\mathfrak{L}_{\mathrm{c}}}$ intersects the boundary of $B$. By Definition 14 for any solution $X$ of $\operatorname{Ball}(P)$, we have that the points of the pair $\Omega_{P}(X)$ are in $\overline{\mathfrak{L}_{\mathrm{n}}} \cup \overline{\mathfrak{L}_{\mathrm{c}}}$ and hence 
are not on the boundary of $B$, which implies that Assumption $\aleph_{2}$ is satisfied. Assume that Assumption $\aleph_{3}$ is not satisfied, that is, there exist two distinct non-twin solutions $X, X^{\prime}$ that have the same plane projection $p \in \mathbb{R}^{2}$. Let $\left(q_{1}, q_{2}\right)=\Omega_{P}(X)$ and $\left(q_{1}^{\prime}, q_{2}^{\prime}\right)=\Omega_{P}\left(X^{\prime}\right)$. By Lemma 13 , the pairs $\left(q_{1}, q_{2}\right),\left(q_{1}^{\prime}, q_{2}^{\prime}\right)$ are distinct and the points $q_{1}, q_{2}, q_{1}^{\prime}, q_{2}^{\prime}$ have the same plane projection $p$. By Assumption $\mathcal{A}_{3}$, we cannot have three pairwise distinct points among $q_{1}, q_{2}, q_{1}^{\prime}, q_{2}^{\prime}$. Moreover, if the multiplicity at all of the points $q_{1}, q_{2}, q_{1}^{\prime}, q_{2}^{\prime}$ is one, then $\left(q_{1}, q_{2}\right),\left(q_{1}^{\prime}, q_{2}^{\prime}\right)$ are in $\widehat{\mathfrak{L}}_{\mathrm{n}}$ and not distinct. Hence, at least a point say $q_{1}$ has multiplicity larger than one, i.e., $q_{1} \in \mathfrak{L}_{\mathrm{c}}$ (Lemma 20p. Hence, the number of solutions counted with multiplicity is at least three which contradicts Assumption $\mathcal{A}_{3}$ Hence, Assumption $\aleph_{3}$ is satisfied.

Now, assume that Assumptions $\aleph_{1}, \aleph_{2}$ and $\aleph_{3}$ are satisfied. Since, by Assumption $\aleph_{1}$, Ball $(P)$ is a regular square system, its solution set is a zero-dimensional manifold in the compact set $\bar{B}_{\mathrm{Ball}(P)}$ (regular value theorem). Hence, Ball $(P)$ has a finite number of solutions in $\bar{B}_{\text {Ball }}$. Since $\Omega_{P}$ (Definition 14) is surjective (Lemma 15), the set $\widehat{\mathfrak{L}}$ (Definition 12 is also finite. Hence, the set $\mathfrak{L}_{\mathrm{c}} \cup \mathfrak{L}_{\mathrm{n}}$ is finite (since $\mathfrak{L}_{\mathrm{c}} \cup \mathfrak{L}_{\mathrm{n}}$ is the image of $\widehat{\mathfrak{L}}$ under the surjective function $\left.\left(q_{1}, q_{2}\right) \rightarrow q_{1}\right)$. Moreover, by Assumption $\aleph_{2}$, the set $\overline{\mathfrak{L}_{\mathrm{n}}} \cup \overline{\mathfrak{L}_{\mathrm{c}}}$ does not intersects the boundary of $B$. Hence, Assumptions $\mathcal{A}_{2}$ and $\mathcal{A}_{4}$ are satisfied in $\bar{B}$. To prove that Assumption $\mathcal{A}_{3}$ is satisfied, let $p=(\alpha, \beta) \in \pi_{\mathfrak{C}}(\mathfrak{C})$ and $\left|\pi^{-1}(p)\right| \geqslant 3$. For pairwise distinct points $q_{1}, q_{2}, q_{3} \in \pi^{-1}(p)$, by Lemma 13 , we have that there exist two distinct non-twin solutions $X, X^{\prime}$ of $\operatorname{Ball}(P)$, with $\Omega_{P}(X)=\left(q_{1}, q_{2}\right)$ and $\Omega_{P}\left(X^{\prime}\right)=\left(q_{1}, q_{3}\right)$ such that we have the same plane projection $p$ which contradicts Assumption $\aleph_{3}$ Hence, $\pi_{\mathfrak{C}}^{-1}(p)$ consists of at most two distinct points. We consider two cases:

(a) $\pi_{\mathfrak{C}}^{-1}(p)$ has two distinct elements, say $q_{1}, q_{2}$. By Lemma 13 , the pair $\left(q_{1}, q_{2}\right)$ is in $\widehat{\mathfrak{L}}_{\mathrm{n}}$, and hence, there exists a solution $X=(\alpha, \beta, y, r, t) \in \mathbb{R} \times \mathbb{R} \times \mathbb{R}^{n-2} \times \mathbb{R}^{n-2} \times \mathbb{R}$ of $\operatorname{Ball}(P)$, with $t \neq 0$ and $\Omega_{P}(X)=\left(q_{1}, q_{2}\right)$. Since $X$ is a regular solution (Assumption $\aleph_{1}$, by Lemma 32 we have that none of $q_{1}, q_{2}$ is in $\mathfrak{L}_{\mathrm{c}}$. Hence, by Lemma 20, the multiplicity of $\left\{P\left(x_{1}, x_{2}, y\right)=0 \in \mathbb{R}^{n-1}, x_{1}-\alpha=x_{2}-\beta=0\right\}$ at $q_{1}$ (resp. $\left.q_{2}\right)$ is one. Thus, the number of solutions counted with multiplicity is two.

(b) $\pi_{\mathfrak{C}}^{-1}(p)$ has a unique point $q$. Let $m$ denote the multiplicity of the system $\left\{P\left(x_{1}, x_{2}, y\right)=0 \in \mathbb{R}^{n-1}, x_{1}-\right.$ $\left.\alpha=x_{2}-\beta=0\right\}$ at $q$. If $m=1$, then we are done. If $m>1$, then by Lemma 20 we have that $q \in \mathfrak{L}_{\mathrm{c}}$. Hence, there exists a solution of $\operatorname{Ball}(P)$ of the form $X=(\alpha, \beta, y, r, 0) \in \mathbb{R} \times \mathbb{R} \times \mathbb{R}^{n-2} \times \mathbb{R}^{n-2} \times \mathbb{R}$ such that $\Omega_{P}(X)=(q, q)$ (Lemma 15). Since $X$ is regular (Assumption $\aleph_{1}$, by Lemma 32 we have that $(q, q)$ satisfies assumption $\mathcal{A}_{5}^{\prime}$. In particular, the multiplicity $m$ is equal to two.

Thus, for all $p \in \pi_{\mathfrak{C}}(\mathfrak{C})$ the sum of the multiplicities of the solutions in the system $\left\{P(x)=0 \in \mathbb{R}^{n-1}, x_{1}-\alpha=\right.$ $\left.x_{2}-\beta=0\right\}$ is at most two, i.e., Assumption $\mathcal{A}_{3}$ is satisfied. Now, since Assumptions $\mathcal{A}_{1}, \mathcal{A}_{2}, \mathcal{A}_{3}$ and $\mathcal{A}_{4}$ are satisfied and since all solutions of $\operatorname{Ball}(P)$ are regular, by Theorem 27 , we have that Assumption $\mathcal{A}_{5}^{-}$is also satisfied.

Using Lemma 35 , we are ready to check Assumptions $\mathcal{A}_{2}, \mathcal{A}_{3}, \mathcal{A}_{4}$ and $\mathcal{A}_{5}^{-}$using $\aleph_{1}, \aleph_{2}$ and $\aleph_{3}$, Since Lemma 35 requires Assumption $\mathcal{A}_{1}$, we start by checking that assumption with Semi-algorithm 1 that is based on 
subdivision.

Semi-algorithm 1 Checking Assumption $\mathcal{A}_{1}$

Input: An open $n$-box $B$ and a function $P$ from $\bar{B}$ to $\mathbb{R}^{n-1}$.

Termination: If and only if $P$ satisfies Assumption $\mathcal{A}_{1}$ in $\bar{B}$.

Output: True.

$$
\begin{aligned}
& \text { 1: } L:=\{\bar{B}\} \\
& \text { 2: } \text { while } L \neq \emptyset \text { do } \\
& \text { 3: } \quad \mathfrak{B}:=\operatorname{pop}(L) \\
& \text { 4: } \quad \text { if } 0 \in \square P(\mathfrak{B}) \text { and } \operatorname{rank}\left(\square J_{P}(\mathfrak{B})\right)<n-1 \text { then } \\
& \text { 5: } \quad \text { Subdivide } \mathfrak{B} \text { and add its children to } L . \\
& \text { 6: } \text { return True. }
\end{aligned}
$$

Lemma 36. Semi-algorithm 1 stops if and only if $P$ satisfies Assumption $\mathcal{A}_{1}$ in $\bar{B}$.

Proof. If Semi-algorithm 1 stops, by the conditions in Step (4), the box $\bar{B}$ is partitioned into two sets of boxes. A set of boxes that are disjoint with $\overline{\mathfrak{C}}$ and the other one is a set of boxes that contain parts of $\overline{\mathfrak{C}}$ that satisfy Assumption $\mathcal{A}_{1}$. Thus, Assumption $\mathcal{A}_{1}$ is satisfied in $\bar{B}$. On the other hand, assume that $P$ satisfies Assumption $\mathcal{A}_{1}$ in $\bar{B}$ and Semi-algorithm 1 does not stop, then, for every positive real $\epsilon$ there exists a closed box $\overline{\mathfrak{B}}_{\epsilon} \subset \bar{B}$, with $w\left(\overline{\mathfrak{B}}_{\epsilon}\right)<\epsilon$ such that the conditions in Step (4) are satisfied in $\overline{\mathfrak{B}}_{\epsilon}$. Consider the infinite chain $\overline{\mathfrak{B}}_{\frac{1}{1}}, \overline{\mathfrak{B}}_{\frac{1}{2}}, \overline{\mathfrak{B}}_{\frac{1}{3}} \ldots$ and take $q_{k} \in \mathfrak{B}_{\frac{1}{k}}$, with $q_{k} \neq q_{k^{\prime}}$ for $k \neq k^{\prime}$. Since $\bar{B}$ is compact, then there exists a subsequence of $q_{k}$ that converges to a point on $\bar{B}$ say $q$. Since $\square P$ and $\square J_{P}$ are box function we must have that $P(q)=0$ and $\operatorname{rank}\left(J_{P}(q)\right)<n-1$. Thus, $q$ is a point in $\overline{\mathfrak{C}}$ that does not satisfy Assumption $\mathcal{A}_{1}$ which is a contradiction. Hence, Semi-algorithm 1 stops.

The next step is to check Assumptions $\aleph_{1}$ and $\aleph_{2}$. For this goal, we want to find a finite set of pairwise disjoint boxes in $\bar{B}_{\text {Ball }}$ such that every box contains at most one solution of $\operatorname{Ball}(P)$ and the union of these boxes contains all solutions of $\operatorname{Ball}(P)$ in $\bar{B}_{\text {Ball }}$. Notice that, by the definition of box functions, for a closed $(2 n-1)$-box $\overline{\mathfrak{U}}$, if $0 \notin \square \operatorname{Ball}(P)(\overline{\mathfrak{U}})$, then $\overline{\mathfrak{U}}$ does not contain a solution of $\operatorname{Ball}(P)$, whereas the condition $0 \in \square \operatorname{Ball}(P)(\overline{\mathfrak{U}})$ does not necessarily imply that a solution is in $\overline{\mathfrak{U}}$. This is why the set we are going to find might have unnecessary boxes. However, we will see later that this is enough for our purpose. Before introducing Semi-algorithm 2, we define the following functions.

Definition 37. Consider the set $\mathbb{R}_{t \geqslant 0}^{2 n-1}=\left\{\left(x_{1}, x_{2}, y, r, t\right) \in \mathbb{R} \times \mathbb{R} \times \mathbb{R}^{n-2} \times \mathbb{R}^{n-2} \times \mathbb{R} \mid t \geqslant 0\right\}$ and define

$$
\begin{aligned}
f_{\text {Ball }}^{ \pm}: \mathbb{R}_{t \geqslant 0}^{2 n-1} & \rightarrow \mathbb{R}^{n} \\
\left(x_{1}, x_{2}, y, r, t\right) & \mapsto\left(x_{1}, x_{2}, y \pm r \sqrt{t}\right)
\end{aligned}
$$

Define the function $f_{\mathrm{Ball}}: \mathbb{R}_{t \geqslant 0}^{2 n-1} \rightarrow \mathbb{R}^{n} \times \mathbb{R}^{n}$ that maps $X$ to $\left(f_{\mathrm{Ball}}^{+}(X), f_{\mathrm{Ball}}^{-}(X)\right)$. Notice that $f_{\mathrm{Ball}}$ is an extension of $\Omega_{P}$ (Definition 14). By abuse of notation, for a set $S \subset \mathbb{R}^{2 n-1}$, we define $f_{\mathrm{Ball}}(S)$ as $f_{\mathrm{Ball}}\left(S \cap \mathbb{R}_{t \geqslant 0}^{2 n-1}\right)$. 
Semi-algorithm 2 Isolating the solutions of $\operatorname{Ball}(P)$ (under Assumption $\mathcal{A}_{1}$

Input: An open $n$-box $B$, a function $P$ from $\bar{B}$ to $\mathbb{R}^{n-1}$ such that $P$ satisfies Assumption $\overline{\mathcal{A}}_{1}$ in $\bar{B}$ and a $(2 n-1)$ open box $\mathfrak{U}_{0}$ that contains $\bar{B}_{\text {Ball }}$ (see Remark 40 ).

Termination: If and only if Ball $(P)$ satisfies Assumptions $\aleph_{1}$ and $\aleph_{2}$ in $\bar{B}_{\text {Ball }}$.

Output: A list of pairwise disjoint isolating boxes of the solutions of Ball $(P)$ in $\mathfrak{U}_{0}$ such that their images by $f_{\text {Ball }}$ lies in $B \times B$.

1: Solutions $=\emptyset$.

2: $L:=\left\{\mathfrak{U}_{0}\right\}$.

3: while $L \neq \emptyset$ do

4: $\quad \mathfrak{U}:=\operatorname{pop}(L)$.

5: $\quad$ if $0 \notin \square \operatorname{Ball}(P)(\overline{\mathfrak{U}})$ or $\left(\square f_{\text {Ball }}(\overline{\mathfrak{U}})\right) \cap(\bar{B} \times \bar{B})=\emptyset$ then

6: $\quad$ Do nothing $(\mathfrak{U}$ is simply removed from $L)$.

7: $\quad$ else if $\operatorname{rank}\left(\square J_{\operatorname{Ball}(P)}(\overline{\mathfrak{U}})\right)=2 n-1$ and $\square f_{\text {Ball }}(\epsilon \text {-inflation }(\overline{\mathfrak{U}}))^{2} \subset B \times B$ then

8: $\quad$ if $\epsilon$-inflation $(\mathfrak{U})$ contains a solution of $\operatorname{Ball}(P)$ (see Remark 38) then

9: $\quad$ Add $\epsilon$-inflation $(\mathfrak{U})$ to Solutions.

10: else

11: $\quad$ Subdivide $\mathfrak{U}$ and add its children to $L$.

12: Remove duplicates in Solutions (see Remark 38).

13: return Solutions

Remark 38. Steps (8) and (12) are not detailed because they are standard in subdivision algorithms to handle the issue of solutions on or near box boundaries and ensuring that solution boxes are pairwise disjoint. We only sketch below how these steps are done and refer to Sta95, \$5.9.1; Kea97: XY19] for details. In Step (8), an existence test is performed by evaluating an interval Newton operator on an $\epsilon$-inflation of the box $\mathfrak{U}$. The inflated box $\epsilon$-inflation( $(\mathfrak{U})$ is certified to contain a solution if its image by the interval Newton operator is contained in the interior of $\epsilon$-inflation( $\mathfrak{U})$. When the existence test is positive, the solution may be on the boundary or even outside $\mathfrak{U}$, but still in the interior of $\epsilon$-inflation( $\mathfrak{U})$. The side effect is that the same solution may be reported several times when it is on or near a boundary of the subdivision. This issue is then solved in Step (12) as follows. When two boxes in the set Solutions intersect, they must report the same solution, and in addition, this solution is in the intersection of the two boxes. In Step (12), we thus compute intersections between boxes and replace intersecting ones by their intersection box. The boxes in the output set Solutions are thus pairwise disjoint.

Lemma 39. Under Assumption $\mathcal{A}_{1}$ in $\bar{B}$, if Semi-algorithm 2 stops, it returns a list of pairwise disjoint isolating boxes of the solutions of $\mathrm{Ball}(P)$ in $\mathfrak{U}_{0}$ such that their images by $f_{\mathrm{Ball}}$ lies in $B \times B$. Moreover, Semi-algorithm 2

\footnotetext{
${ }^{2}$ For a box $\mathfrak{U}$ and $\epsilon>0, \epsilon$-inflation( $\left.\mathfrak{U}\right)$ is the box that has the same center as $\mathfrak{U}$ and its width is that of $\mathfrak{U}$ multiplied by $(1+\epsilon)$. The box $\epsilon$ inflation( $(\mathfrak{U})$ thus contains $\mathfrak{U}$, the exact value of $\epsilon$ is not important for the algorithm and it is usually set to 0.1 in subdivision algorithms $\mid$ Rum 10$]$.
} 
stops if and only if $\mathrm{Ball}(P)$ satisfies Assumptions $\aleph_{1} \aleph_{2}$ in $\bar{B}_{\text {Ball. }}$

Proof. We first prove the correctness of the Semi-algorithm 2 assuming that it terminates. Since Step (5) is the only time the algorithm discards boxes, it never discards a box that contains a solution of $\operatorname{Ball}(P)$ in $\mathfrak{U}_{0}$ such that its image by $f_{\text {Ball }}$ lies in $B \times B$. Hence, all such solutions of $\operatorname{Ball}(P)$ lie in output boxes. The rank condition in Step (7) guarantees that each output box contains at most one solution of Ball $(P)$ [Sny92b, Theorem A.1]. The fact that every output box contains at least one solution is ensured by a standard algorithm in Step (8) (see e.g., Neu91, Theorem 5.6.2; XY19] and Remark 38). Finally, by Step (12), the output boxes are pairwise disjoint, hence the algorithm outputs isolating boxes of the solutions of $\operatorname{Ball}(P)$ in $\mathfrak{U}_{0}$ such that their images by $f_{\text {Ball }}$ lie in $B \times B$.

To prove the equivalence for the termination, first assume that Semi-algorithm 2 stops and returns Solutions. According to the correctness proof, every solution $X$ of $\operatorname{Ball}(P)$ in $\bar{B}_{\text {Ball }}$ is regular and satisfies $\Omega_{P}(X) \in B \times B$. Thus, Assumptions $\aleph_{1}$ and $\aleph_{2}$ are satisfied in $\bar{B}_{\text {Ball }}$.

On the other hand, assume that $\aleph_{1}$ and $\aleph_{2}$ hold in $\bar{B}_{\text {Ball }}$. We prove that Semi-algorithm 2 terminates. By Assumption $\aleph_{1}$ all solutions in $\bar{B}_{\text {Ball }}$ of the square system $\operatorname{Ball}(P)$ are regular. Hence, they form a zero-dimensional manifold in the compact space $\bar{B}_{\text {Ball }}$. Thus, the solution set is finite. We now prove that for any box $\overline{\mathfrak{U}} \in L$ with a small enough width, one of the conditions in Step (5) or the conditions in Steps (7-8) are satisfied. Thus, in both cases $\overline{\mathfrak{U}}$ will be removed from $L$, and hence, Semi-algorithm 2 stops after a finite number of iterations. Due to Assumption $\aleph_{2}$, after a finite number of iterations, no box $\mathfrak{U}$ in $L$ intersects the boundary of $B \times B$. Moreover, due to the convergence of the box evaluations, we can also assume that either $\square f_{\text {Ball }}(\epsilon$-inflation $(\overline{\mathfrak{U}})) \subset B \times B$, which is the second condition of Step (7), or $\left(\square f_{\text {Ball }}(\overline{\mathfrak{U}})\right) \cap(\bar{B} \times \bar{B})=\emptyset$ which is the second condition of Step (5).

If $\mathfrak{U}$ does not contain a solution of $\operatorname{Ball}(P)$, then due to convergence of the box function evaluation of $\operatorname{Ball}(P)$, after a finite number of iterations, every children $\mathfrak{U}^{\prime}$ of $\mathfrak{U}$ satisfies $0 \notin \square \operatorname{Ball}(P)\left(\overline{\mathfrak{U}^{\prime}}\right)$, that is, it is discarded in Step (5).

If $\mathfrak{U}$ contains a solution of $\operatorname{Ball}(P)$ in $\bar{B}_{\text {Ball }}$, according to Assumption $\aleph_{1}$ it is a regular solution. Due to the convergence of the box evaluation $\operatorname{det}\left(J_{\mathrm{Ball}(P)}(\overline{\mathfrak{U}})\right)$ will eventually be non zero and thus $\operatorname{rank}\left(\square J_{\operatorname{Ball}(P)}(\overline{\mathfrak{U}})\right)$ will eventually be $2 n-1$ after a finite number of iterations, which is the first condition of Step (7). Due to the convergence of the interval Newton existence test, the condition of Step (8) will also be eventually satisfied (see Remark 38). The refined box will then eventually be added in the Solutions list.

Thus, for any box in $L$ with a small enough width, one of the conditions of Step (5) is satisfied or all of the conditions in Step (7-8) are satisfied, thus it is either discarded or added to the output. Hence, Semi-algorithm 2 terminates.

Remark 40. Semi-algorithm 2 requires a closed $(2 n-1)$-box $\overline{\mathfrak{U}}_{0}$ that contains $\bar{B}_{\text {Ball. }}$. For instance the following set could be used: $\left\{(q, r, t) \in \mathbb{R}^{2 n-1} \mid q \in \bar{B},-1 \leqslant r_{i} \leqslant 1\right.$ for $\left.i \in\{3, \ldots, n\}, 0 \leqslant t \leqslant \frac{\xi^{2}}{4}\right\}$ with $\xi=$ $\max \left\{\left\|q-q^{\prime}\right\| \mid q, q^{\prime} \in \bar{B}\right\}$.

Finally, using Lemma 35 Semi-algorithm 3 checks whether $P$ satisfies Assumptions $\mathcal{A}_{1}, \mathcal{A}_{2}, \mathcal{A}_{3}, \mathcal{A}_{4}$ and $\mathcal{A}_{5}^{-}$ 
in $\bar{B}$ and outputs a superset of isolating boxes of the singularities of $\pi_{\mathfrak{C}}(\mathfrak{C})$.

Semi-algorithm 3 Checking the weak assumptions and computing a superset of the singularities of $\pi_{\mathfrak{C}}(\mathfrak{C})$

Input: An open $n$-box $B$ and a smooth function $P$ from $\bar{B}$ to $\mathbb{R}^{n-1}$.

Termination: If and only if $P$ satisfies Assumptions $\mathcal{A}_{1}, \mathcal{A}_{2}, \mathcal{A}_{3}, \mathcal{A}_{4}$ and $\mathcal{A}_{5}^{-}$in $\bar{B}$.

Output: $N$, a list of certified node singularities: a list of boxes in $\mathbb{R}^{2 n-1}$ whose projections in $\mathbb{R}^{2}$ contain each a single node of $\pi_{\mathfrak{C}}(\mathfrak{C})$.

$U$, a list of uncertified singularities: a list of boxes in $\mathbb{R}^{2 n-1}$ whose projections in $\mathbb{R}^{2}$ contain each at most one node or one cusp of $\pi_{\mathfrak{C}}(\mathfrak{C})$.

The union of all these projected 2D boxes contains all the singularities of $\pi_{\mathfrak{C}}(\mathfrak{C})$.

1: Check Assumption $\mathcal{A}_{1}($ Semi-algorithm 1 ).

2: Compute a closed $(2 n-1)$-box $\overline{\mathfrak{U}}_{0}$ that contains $\bar{B}_{\text {Ball }}($ Remark 40 .

3: $L:=$ output of Semi-algorithm 2

4: Keep refining all boxes $\overline{\mathfrak{U}} \in L$ (see Remark 41). until no triplets of boxes overlap in projection. Then remove from $L$ one box from every pair (see Remark 17). This ensures Assumption $\aleph_{3}$

5: $N:=$ boxes of $L$ that lie in the halfspace $t>0$.

6: $U:=$ boxes of $L$ that intersect the hyperplane $t=0$.

7: return $N$ and $U$.

Remark 41. The refinement of an isolating box of a solution is performed by iterative evaluation of an interval Newton operator; we refer to [Neu91] Theorem 5.6.2] for details.

To identify the possible cusp points in the set $U$ returned by Semi-algorithm 3 , one may wish to solve independently the Ball system with the additional constraint $t=0$ (by Remark 16). Unfortunately, in this case we have an over-determined system and thus we cannot certify its solutions numerically. In the special case of a silhouette curve, it is possible to identify cusp points with numerical algorithms in the case $n=3$ [IMP16a, Lemmas $9 \&$ 10], but we leave as a conjecture its generalization for $n>3$.

On the other hand, for curves that satisfy the strong assumptions, $\mathcal{A}_{5}$ ensures that there are no cusps in the projection, which is equivalent to $\widehat{\mathfrak{L}}_{\mathrm{c}}$ being empty and $\operatorname{Ball}(P)$ having no solutions on the hyperplane $t=0$ (by Remark 16. Hence, if Assumptions $\mathcal{A}_{1-5}$ hold, we can refine the boxes output by Semi-Algorithm 3 until no box intersects $t=0$. Boxes in the half-space $t<0$ correspond to imaginary points in $\mathbb{C}^{n}$ (Definition 14). Then the boxes satisfying $t>0$ are all the isolating boxes of the nodes of $\pi_{\mathfrak{C}}(\mathfrak{C})$ by Lemmas 39 and 13 , Remark 16 and Lemma 26

Semi-algorithm 4 Checking Assumptions $\left|\mathcal{A}_{1-5}\right|$ and isolating the singularities of $\pi_{\mathfrak{C}}(\mathfrak{C})$

Input: An open $n$-box $B$ and a smooth function $P$ from $\bar{B}$ to $\mathbb{R}^{n-1}$.

Termination: If and only if $P$ satisfies Assumptions $\mathcal{A}_{1-5}$ in $\bar{B}$. 
Output: A list of boxes in $\mathbb{R}^{2 n-1}$ whose projections in $\mathbb{R}^{2}$ are isolating boxes of the singularities $\pi_{\mathfrak{C}}(\mathfrak{C}$ ) (all singularities are in some boxes and each box contains a unique singularity).

1: $N, U:=$ output of Semi-Algorithm 3

2: for all $\overline{\mathfrak{U}} \in U$ do

3: $\quad$ Keep refining $\overline{\mathfrak{U}}$ (see Remark 41) until it does not intersect the hyperplane $t=0$ and discard it if it lies in the half-space $t<0$.

4: return $N \cup U$.

\section{Implementation and experiments}

We first describe, in Section 6.1 the algorithms we implemented in our software Isolating_singularities $\sqrt[3]{ }$ with, in particular, the refinements we considered for improving the running time of those presented in Section 5 . We present in Section 6.2 the third-party libraries we use. In Section 6.3 we present our experiments on several analytic curves in 3 and 4 dimensions and discuss the efficiency of our implementation.

\subsection{Algorithms}

Semi-algorithms 3 and 4 of Section 5 take as input an open $n$-box $B$ and a curve $\mathfrak{C}$ defined by a smooth function $P$ from $\bar{B}$ to $\mathbb{R}^{n-1}$. They terminate if and only if $P$ satisfies our weak, respectively strong, assumptions of Definition 8 . Upon termination, they output a superset of the singularities, respectively the singularities, of the curve $\pi_{\mathfrak{C}}(\mathfrak{C})$.

The algorithms we implemented are variants of Semi-algorithms 3 and 4 . First, for visualization purposes we modified their output as follows. Instead of returning boxes in $\mathbb{R}^{2 n-1}$ isolating solutions of the Ball system, they now return their projections in $\mathbb{R}^{2}$ that contain singularities of $\pi_{\mathfrak{C}}(\mathfrak{C})$. In addition, they also return the projection in $\mathbb{R}^{2}$ of the boxes enclosing the curve $\mathfrak{C}$, that thus enclose the curve $\pi_{\mathfrak{C}}(\mathfrak{C})$.

The two main improvements of our implementations are described below. The first one solves a stability issue when singular points of the projection are induced by very close branches of the curve $\mathfrak{C}$ or by a close to vertical part of the curve. The second one is a generalization of an idea used in the three-dimensional case in [IMP18], it aims at reducing the domain where the Ball system is to be solved. It is critical for the efficiency since solving in this high-dimensional space is costly.

Evaluating the operators $S$ and D of Definition 9 To solve the Ball system we need box functions for the operators $S$ and $D$. We first note that if $P\left(x_{1}, x_{2}, y\right) \in C^{\infty}\left(\mathbb{R}^{n}, \mathbb{R}\right)$ is a polynomial function, $S \cdot P$ (resp. $D \cdot P$ ) is also a polynomial function and can be computed from the terms of $P\left(x_{1}, x_{2}, y+r t\right)$ that have even (resp. odd) exponents in the variable $t$, see [IMP16b, Lemma 6] for details.

$3^{\text {https://github.com/gkrait/Isolating_singularities }}$ 
If $P$ is a more general $C^{\infty}$ function for which we have a box function, using Definition 9 , computing $S \cdot P$ on any $(2 n-1)$-box $\overline{\mathfrak{U}}$ or computing $D \cdot P$ on $\overline{\mathfrak{U}}$ such that its $t$-interval does not contain 0 , is implemented from the box function of $P$ and interval arithmetic. On the other hand, when the $t$-interval contains 0 or is close to 0 , the division by $\sqrt{t}$ in the formula for $D \cdot P$ makes the computation undefined or unstable. In such non-polynomial cases, we use a Taylor form at order 3 [Ral83], that is, we compute a Taylor expansion with remainder at $t=0$ of $D \cdot P$ and evaluate by interval the third order derivative. We define a threshold $\delta_{\text {Taylor }}$ (which we set to $10^{-2}$ in our experiments) such that this Taylor form is used when the $t$-interval has values smaller than $\delta_{\text {Taylor }}$.

Improvement of Semi-algorithm 3 The domain $B_{\text {Ball }}$ where the Ball system is solved is refined to reduce costly computations in the high-dimensional space $\mathbb{R}^{2 n-1}$ by first enclosing the curve $\mathfrak{C}$ in a union of boxes in the smaller space $\mathbb{R}^{n}$. We denote this set of boxes by enclosing_curve. Our approach follows the observation that every cusp of $\pi_{\mathfrak{C}}(\mathfrak{C})$ lies in the projection of a box of enclosing_curve containing a point $p$ of $\mathfrak{C}$ with a tangent orthogonal to the $\left(x_{1}, x_{2}\right)$-plane. Such a point $p$ is both $x_{1}$ and $x_{2}$-critical for $\mathfrak{C}$, that is, both $\operatorname{det}\left(M_{1}(p)\right)$ and $\operatorname{det}\left(M_{2}(p)\right)$ vanish $\left(M_{i}\right.$ denotes the minor of $J_{P}$ obtained by removing the $i$-th column). In addition, every node in $\pi_{\mathfrak{C}}(\mathfrak{C})$ is contained in:

(a) the projection of a box $\mathfrak{B}$ in enclosing_curve such that $0 \in \operatorname{det}\left(\square M_{1}\right)(\mathfrak{B})$ and $0 \in \operatorname{det}\left(\square M_{2}\right)(\mathfrak{B})$, or

(b) the intersection of the plane projections of two boxes in enclosing_curve.

To understand this observation, we say that $\mathfrak{C}$ is parameterizable by $x_{i}$ in a box $\mathfrak{B}$, if for any particular value $\alpha$ of $x_{i}$ in $\mathfrak{B}$, the hyperplane $x_{i}=\alpha$ intersects the curve $\mathfrak{C}$ at most once in $\mathfrak{B}$. The interval implicit function theorem [Sny92a, Thm C5 p.291] states that a sufficient condition for $\mathfrak{C}$ to be parameterizable by $x_{i}$ in $\mathfrak{B}$, is that $0 \notin \operatorname{det}\left(\square M_{i}\right)(\mathfrak{B})$. In a box $\mathfrak{B}$ such that $0 \notin \operatorname{det}\left(\square M_{i}\right)(\mathfrak{B})$ for $i=1$ or $2, \mathfrak{C}$ is parameterizable in $x_{i}$, thus $\mathfrak{C}$ cannot contain two points with the same $x_{i}$ value, which implies that the projection of $\mathfrak{C} \cap \mathfrak{B}$ does not contain a node. It follows that such a box can only induce a node in the projection when it overlaps in projection with another box, this case being covered by criterion (b). All nodes or cusps are thus in the projection of boxes satisfying criteria (a) or (b). Using the mapping $f_{\text {Ball }}$ of Definition 37 from the Ball system space $\mathbb{R}^{2 n-1}$ to pairs of points in $\mathbb{R}^{n}$, we only have to solve the Ball system on the pre-image of these particular boxes or pairs of boxes in the set enclosing_curve. More precisely, the Ball system domains associated to boxes in enclosing_curve are defined as follows:

(i) for a single box $\mathfrak{B}$, cross product of boxes $\mathfrak{B}_{\left(x_{1}, x_{2}\right)}$ in $\mathbb{R}^{2}$ and $\mathfrak{B}_{y}$ in $\mathbb{R}^{n-2}$, the domain is $\left\{\left(x_{1}, x_{2}, y, r, t\right) \in \mathbb{R} \times \mathbb{R} \times \mathbb{R}^{n-2} \times \mathbb{R}^{n-2} \times \mathbb{R},\left(x_{1}, x_{2}, y\right) \in \mathfrak{B}, r \in[-1,1]^{n-2}, 0 \leqslant t \leqslant \max \left(\frac{\left\|\mathfrak{B}_{y}-\mathfrak{B}_{y}\right\|^{2}}{4}\right)\right\}$,

(ii) for a pair of boxes $\mathfrak{B}=\mathfrak{B}_{\left(x_{1}, x_{2}\right)} \times \mathfrak{B}_{y}$ and $\mathfrak{B}^{\prime}=\mathfrak{B}_{\left(x_{1}, x_{2}\right)}^{\prime} \times \mathfrak{B}_{y}^{\prime}$, the domain is $\left\{\left(x_{1}, x_{2}, y, r, t\right),\left(x_{1}, x_{2}\right) \in \mathfrak{B}_{\left(x_{1}, x_{2}\right)} \cap \mathfrak{B}_{\left(x_{1}, x_{2}\right)}^{\prime}, y \in \frac{1}{2}\left(\mathfrak{B}_{y}+\mathfrak{B}_{y}^{\prime}\right), r \in[-1,1]^{n-2}\right.$ if $\mathfrak{B} \cap \mathfrak{B}^{\prime} \neq \emptyset$, otherwise, $r \in$ $\left.\frac{\mathfrak{B}_{y}-\mathfrak{B}_{y}^{\prime}}{\left\|\mathfrak{B}_{y}-\mathfrak{B}_{y}^{\prime}\right\|}, t \in \frac{\left\|\mathfrak{B}_{y}-\mathfrak{B}_{y}^{\prime}\right\|^{2}}{4}\right\}$. 
To sum up, our improved versions of Semi-algorithm 3 and 4 consists of three steps: (i) computing a set enclosing_curve of $n$-boxes that enclose the curve $\mathfrak{C}$, (ii) finding the boxes in enclosing_curve that satisfy the above criteria (a) or (b), and (iii) solving $\operatorname{Ball}(P)$ over the pre-image of these boxes under $f_{\text {Ball }}$. When the semialgorithms terminate, they return the 2D projections of the boxes in the set enclosing_curve that cover $\pi_{\mathfrak{C}}(\mathfrak{C})$ together with the boxes that isolate the singularities of $\pi_{\mathfrak{C}}(\mathfrak{C})$.

\subsection{Third-party libraries}

Our software is based on interval arithmetic, interval evaluations of analytic functions and an interval solver. We use the following libraries, Python-FLINT and Ibexsolve, for these tasks.

Python-FLINT is a Python extension module wrapping FLINT (Fast Library for Number Theory) and Arb (arbitrary-precision ball arithmetic), which offers a toolbox for interval arithmetic and evaluation of analytic functions.

Ibexsolve is a $\mathrm{C}^{++}$end-user program that solves systems of non-linear equations rigorously, that is, it does not lose any solution and return each solution under the form of a small box enclosing the true value. It implements a classical branch-and-prune algorithm that interleaves contractions and branching (bisections) to enclose the solutions of a system at any given desired precision. However, as opposed to Arb Ibexsolve has a fixed precision, hence when several solutions are closer to each other than this precision, it will correctly return an enclosing box for these solutions but it will fail at isolating them. In our software, we use the default precision which is $10^{-7}$. Ibexsolve, and thus also our software Isolating_singularities, use a parameter eps_max that defines a maximum width for the isolating boxes output by the solver (the box bisections are forced until all output boxes are not larger than eps_max). We use Ibexsolve for solving the Ball system (Semi-algorithm 2) and also in a variant of Semi-algorithm 1 to check the smoothness of the curve $\mathfrak{C}$ and at the same time enclosing $\mathfrak{C}$ in a set of boxes of $\mathbb{R}^{n}$.

\subsection{Experiments}

In this section, we present four experiments performed with our software Isolating_singularities. More precisely, we applied our improved Semi-algorithm 3 on Experiment 1 and Semi-algorithm 4 on all other experiments. The first example is pedagogical and considers a simple analytic curve in $\mathbb{R}^{3}$ that induces only one node and one ordinary cusp in $\mathbb{R}^{2}$. The second example considers a smooth analytic curve in $\mathbb{R}^{4}$ that induces many nodes in $\mathbb{R}^{2}$. The third one considers sparse but reasonably-high-degree algebraic equations in $\mathbb{R}^{4}$. It should be stressed that, up to our knowledge, the two latter examples are out of reach by other methods: indeed, no other certified algorithm can handle non-algebraic curves in dimension higher than 3 and, for reasonably-high-degree algebraic equations in $\mathbb{R}^{4}$, the bivariate equation defining their $2 \mathrm{D}$ projection often has a very high degree (see Section 6.3 .3 for details).

Finally, in the fourth example, we exhibit the behavior of our software when a node in $\mathbb{R}^{2}$ is induced by a pair of points (on the space curve) that are very close. Indeed, when the equations defining the space curve are not algebraic, the Ball system contains a division by $\sqrt{t}$ (due to the formula of $D \cdot P$ ), which may cause instability 


\begin{tabular}{|c|c|c|c|c|c|c|c|c|}
\hline \multirow[b]{2}{*}{ Experiments } & \multirow[b]{2}{*}{$\begin{array}{l}\text { Boxes } \\
\text { max. } \\
\text { width }\end{array}$} & \multicolumn{3}{|c|}{ Enclosing curve } & \multicolumn{2}{|c|}{$\begin{array}{c}\text { Solving Ball } \\
\text { system }\end{array}$} & \multicolumn{2}{|c|}{ Output } \\
\hline & & Tree size & $\begin{array}{l}\text { Output } \\
\text { boxes }\end{array}$ & Time & Tree size & Time & $\begin{array}{l}\text { Total } \\
\text { time }\end{array}$ & $\begin{array}{c}\text { Singularity } \\
\text { boxes }\end{array}$ \\
\hline \multirow{3}{*}{ Experiment 1} & 0.1 & 535 & 134 & 0.1 & 70 & 3.6 & 3.7 & \multirow{3}{*}{2} \\
\hline & 0.03 & 1835 & 456 & 0.3 & 90 & 3.8 & 4.1 & \\
\hline & 0.01 & 5427 & 1354 & 0.7 & 188 & 4.4 & 5.1 & \\
\hline \multirow{3}{*}{ Experiment 2} & 0.1 & 2243 & 520 & 1.1 & 6098 & 52 & 53.1 & \multirow{3}{*}{43} \\
\hline & 0.03 & 6759 & 1639 & 3.4 & 1078 & 35.4 & 38.8 & \\
\hline & 0.01 & 19583 & 4847 & 10.2 & 372 & 35.8 & 45.6 & \\
\hline \multirow{3}{*}{ Experiment 3} & 0.1 & 1151 & 203 & 1.0 & 655 & 4.2 & 5.2 & \multirow{3}{*}{7} \\
\hline & 0.03 & 2503 & 523 & 1.8 & 272 & 3.5 & 5.3 & \\
\hline & 0.01 & 6347 & 1482 & 4.5 & 163 & 5.7 & 10.2 & \\
\hline
\end{tabular}

Table 1: Running times (in seconds) and numbers of boxes in Experiments 1 to 3.

since $t$ tends to zero when the distance between the pair of points tend to zero. For that purpose, we consider two very close skew lines defined analytically (and not algebraically).

We report the running times and other relevant parameters in Tables 1 and 2 Running times are in seconds on a sequential Intel(R) Core(TM) i7-7600U CPU @ 2.80GHz machine with Linux. We emphasize that the experiments are done with a prototype implementation that is under ongoing development. The tree size columns reports the total number of boxes created during the subdivision algorithm either for enclosing the curve $\mathfrak{C}$ in $\mathbb{R}^{n}$ or for solving the Ball system in $\mathbb{R}^{2 n-1}$. For the enclosing part, the column output boxes is the number of boxes in the set enclosing_curve. For each experiment, we provide a visualization of the plane projected curve $\pi_{\mathfrak{C}}(\mathfrak{C})$ with its singularities. On each figure, the green boxes are the plane projections of the boxes in enclosing_curve that enclose $\mathfrak{C}$, hence these green boxes enclose $\pi_{\mathfrak{C}}(\mathfrak{C})$. The black boxes are the projections of the Ball system solution boxes identifying nodes of the plane curve $\pi_{\mathfrak{C}}(\mathfrak{C})$.

For each experiment, we consider three values of eps_max and it can be observed (see Table 1) that the smaller the value of eps_max, the larger the set enclosing_curve, and the longer it is to compute. As expected, even with the improvement to reduce the Ball system domains to be solved in, the subdivision in the high-dimensional space $\mathbb{R}^{2 n-1}$ is the dominant step of the algorithm.

\subsubsection{Experiment 1: Analytic curve in $\mathbb{R}^{3}$ generating one node and one ordinary cusp}

We start with a pedagogical example pictured in Figure 6 Running times are given in Table 1 . The curve $\mathfrak{C}$ is defined in the box $B=(-1,4) \times(-1,4) \times(-4.8,-1.4)$ by

$$
P\left(x_{1}, x_{2}, x_{3}\right)=\left[x_{1}-\cos \left(x_{3}\right)\left(3+\sin ^{4}\left(x_{3}\right)\right)+3, \quad x_{2}-\sin ^{2}\left(x_{3}\right)\left(3+\sin \left(2 x_{3}\right)\right)\right] .
$$




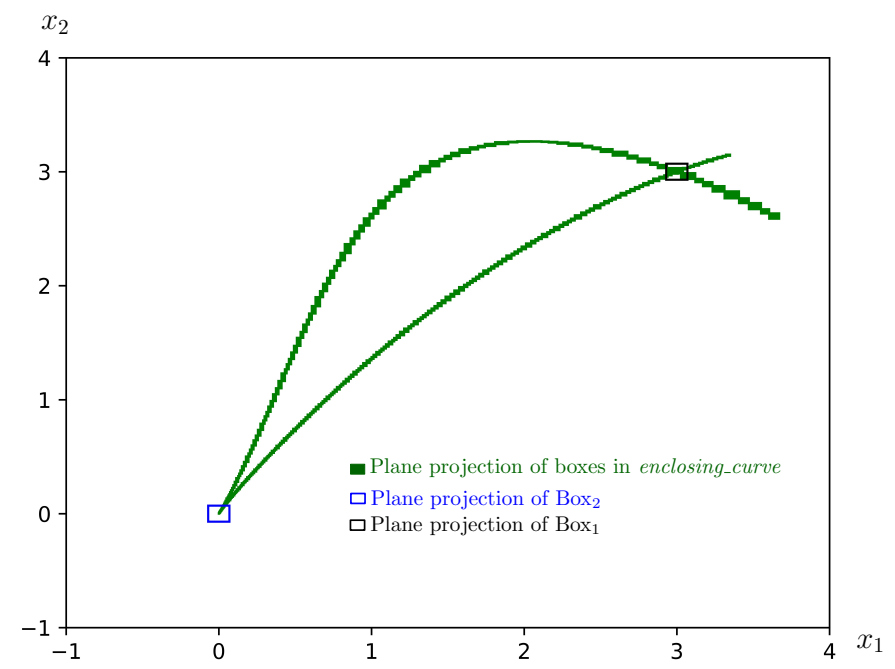

Figure 6: Experiment 1: Plane projection of an analytic curve in $\mathbb{R}^{3}$ with one node and one ordinary cusp.

Our improved Semi-algorithm 3 outputs the following solutions for the Ball system in $\mathbb{R}^{5}$ :

$$
\left\{\begin{array}{l}
N=\left\{\operatorname{Box}_{1}=[3,3] \times[3,3] \times[-3.15,-3.14] \times[1,1] \times[2.4673,2.4675]\right. \\
U=\left\{\operatorname{Box}_{2}=[-0.06,0.04] \times[-0.04,0.07] \times[-3.15,-3.14] \times[1,1] \times[-0.01,0.01]\right\}
\end{array}\right.
$$

The goal of this experiment is to emphasize the genericity of the assumptions and the efficiency of our software in the sparse polynomial case. The curve $\mathfrak{C}$ in defined in the 4-box $B=(-1,0.2) \times(-0.2,1.4) \times(-10,10)^{2}$ and

$$
\begin{aligned}
P= & {\left[x_{1}+2 \sin \left(x_{1}\right)-\cos \left(x_{4}\right)-\left(3 \cos \left(x_{3}\right)-\cos \left(2.8571 x_{3}\right)\right),\right.} \\
& x_{2}+0.2 \cos \left(x_{2}\right)+\left(3 \sin \left(x_{3}\right)-\sin \left(2.8571 x_{3}\right)\right)+\sin \left(x_{4}\right), \\
& \left.x_{4}^{2}-\sin \left(x_{2}\right)\right]
\end{aligned}
$$

over the box $B=(-1,0) \times(-0.1,3.5) \times(-20,20) \times(-10,10)$. This curve has many nodes, some of them very close to each other. Running times are given in Table 1 .

\subsubsection{Experiment 3: High degree algebraic curve in $\mathbb{R}^{4}$}




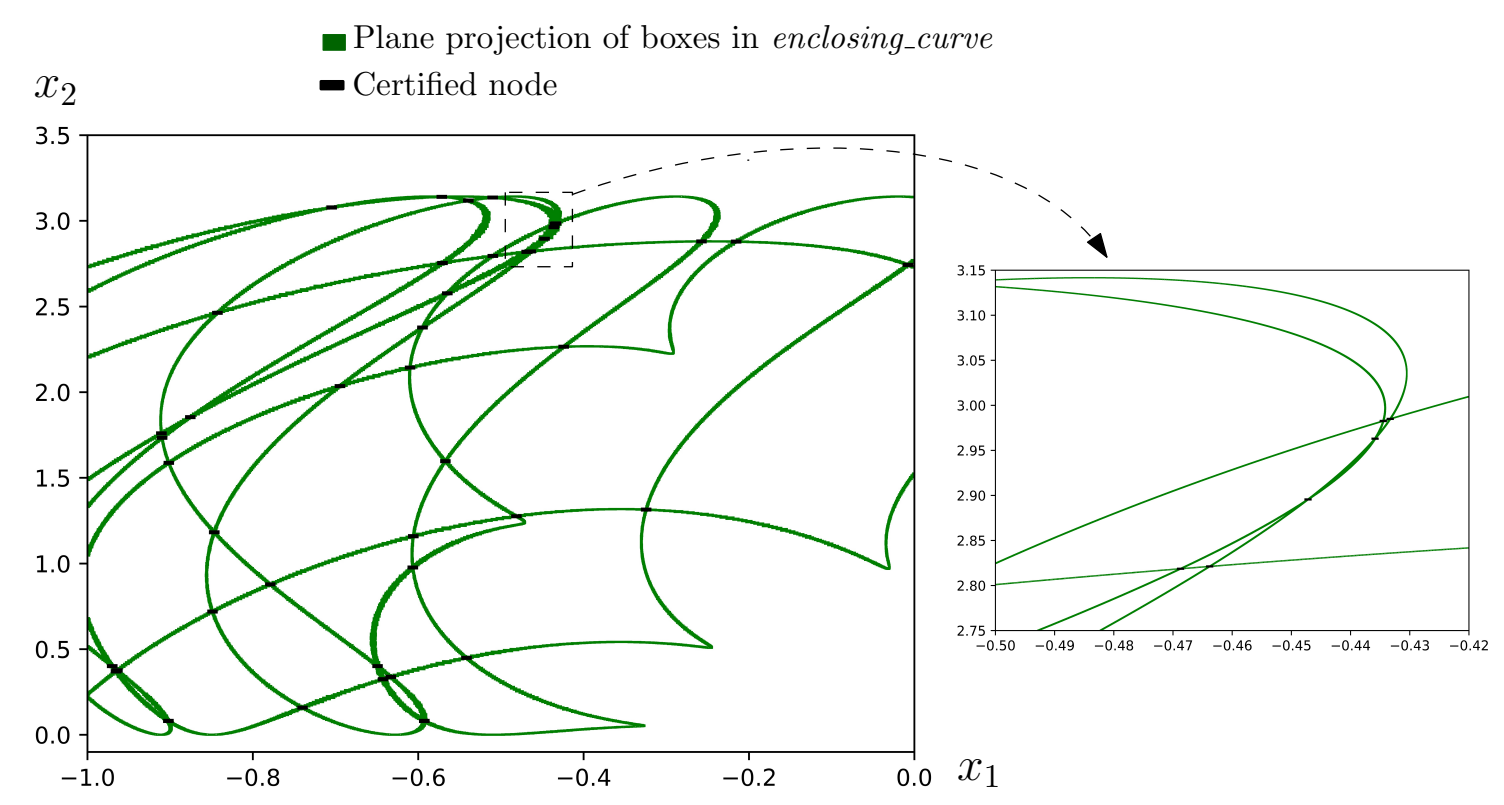

Figure 7: Experiment 2: Plane projection of an analytical curve $\mathfrak{C}$ in $\mathbb{R}^{4}$. Each of the 43 black boxes contains a node of $\pi_{\mathfrak{C}}(\mathfrak{C})$ and is the projection of a box in $\mathbb{R}^{7}$ containing one solution of $\operatorname{Ball}(P)$.

is the zero set of three polynomials of degrees 17,15 and 13, respectively that have a unique monomial of highest degree (which is monic) and 9 other random monomials of degrees at most 2 with integer coefficients in $(-25,25)$.

$$
\begin{aligned}
P= & {\left[x_{1}^{17}-14 x_{1}^{2}-7 x_{1} x_{3}-7 x_{2}^{2}-22 x_{2} x_{4}-x_{3} x_{4}-19 x_{4}^{2}+8 x_{1}-14 x_{3}+9,\right.} \\
& x_{2}^{15}+8 x_{1} x_{3}-14 x_{1} x_{4}-15 x_{2}^{2}+16 x_{2} x_{3}+8 x_{2} x_{4}+2 x_{3}^{2}+13 x_{4}^{2}+11 x_{1}+11 x_{2}, \\
& \left.x_{3}^{13}+17 x_{1}^{2}-15 x_{1} x_{2}+4 x_{1} x_{3}-20 x_{1} x_{4}+2 x_{2}^{2}-10 x_{2} x_{3}+4 x_{2} x_{4}+20 x_{4}^{2}-23 x_{2}\right] .
\end{aligned}
$$

Figure 8 illustrates the 7 nodes of the projection of $\mathfrak{C}$ and running times are given in Table 1 .

Note that since $P$ is polynomial, the implicit equation of $\pi_{\mathfrak{C}}(\mathfrak{C})$ can be computed using elimination theory and its singularities can then be isolated using algebraic solvers. However, the implicit equation we obtained for $\pi_{\mathfrak{C}}(\mathfrak{C})$ is defined by an irreducible bivariate polynomial of degree 442 with 51074 monomials. Isolating the singularities of such a high-degree polynomial is then a real challenge.

Note also that our class of examples is rather specific and our software does not work that well if our defining polynomials are dense or even if they have non-monic high-degree monomials. However, it should be stressed that our software is a prototype and that it would be more efficient to use an interval solver specialized for the algebraic case than the versatile Ibex solver we used. Such a specialized approach has been proved successful in the 3-dimensional case by Imbach et al. [IMP18].

\subsubsection{Experiment 4: Two close lines in $\mathbb{R}^{3}$ generating a node}

As mentioned in the preamble of Section 6.3 the purpose of this experiment $\{13$ is to study the behavior of our 


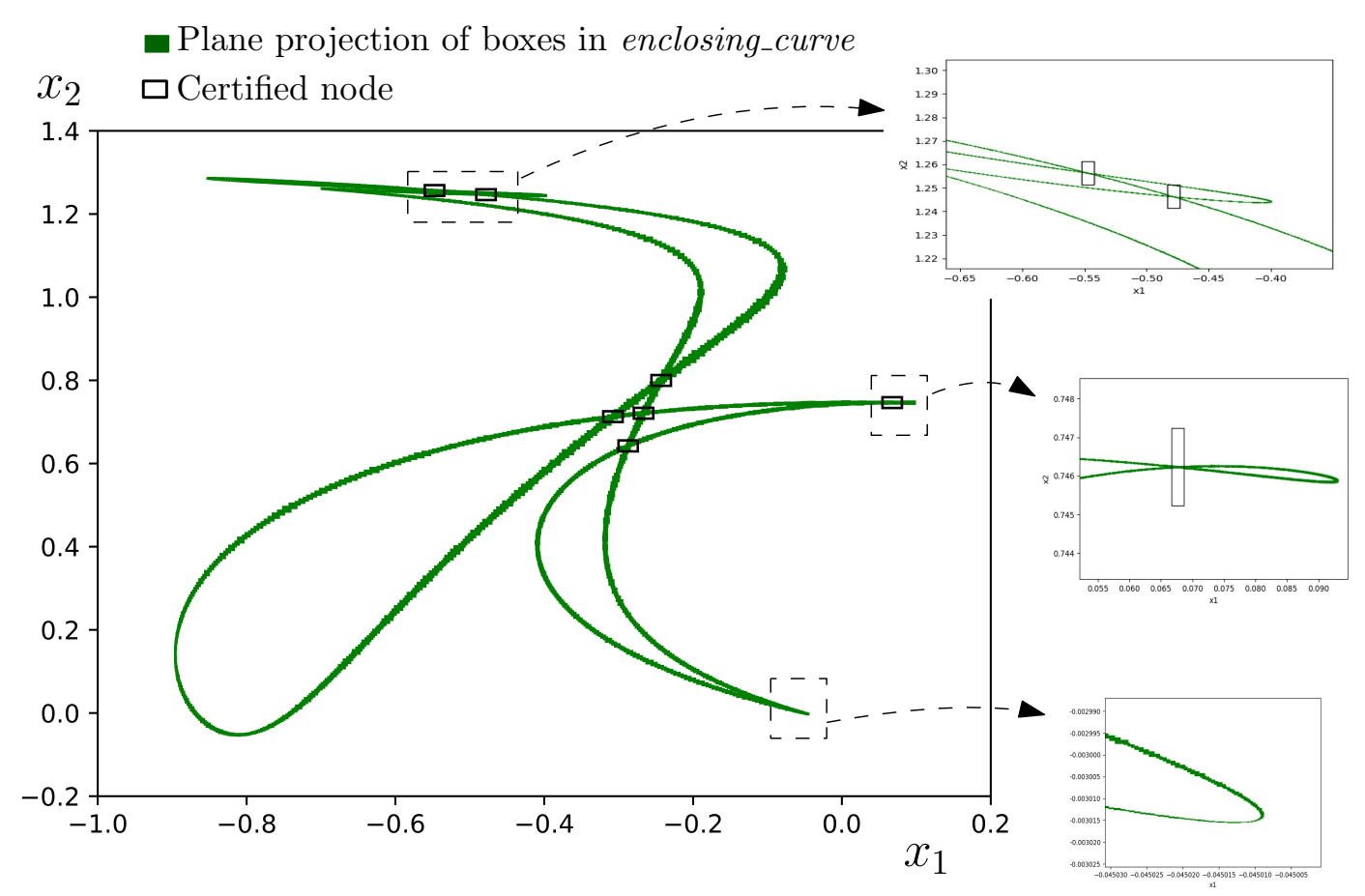

Figure 8: Experiment 3: High degree algebraic curve in $\mathbb{R}^{3}$ generating 7 nodes.

software when a node in $\mathbb{R}^{2}$ is induced by a pair of points (on the space curve) that are very close; namely when a node $\left(x_{1}, x_{2}\right) \in \mathbb{R}^{2}$ is induced by the pair of points $\left(x_{1}, x_{2}, y \pm r \sqrt{t}\right) \in \mathbb{R}^{n}$ with $t$ that tends to zero. Indeed, when the equations defining the space curve are not algebraic, the Ball system contains a division by $\sqrt{t}$ (due to the formula of $D \cdot P$ ), which may cause instability since $t$ tends to zero when the distance between the pair of points tend to zero.

The simplest example to consider is the two skew lines $x_{2}=x_{1}$ in the plane $x_{3}=\epsilon$ and $x_{2}=-x_{1}$ in the plane $x_{3}=-\epsilon$, whose projection in the $\left(x_{1}, x_{2}\right)$-plane has a node at the origin, and to make $\epsilon$ vary towards 0. The pair of lines is thus defined by $\left[\epsilon x_{2}-x_{1} x_{3},\left(x_{3}-\epsilon\right)\left(x_{3}+\epsilon\right)\right]$ but, in order to have non-algebraic equations, we replace $x_{3}$ by $\sin x_{3}$ and define our two lines by $P=\left[\epsilon x_{2}-x_{1} \sin x_{3}, \sin ^{2} x_{3}-\epsilon^{2}\right]$ in the box $B=(-1,1)^{3}$.

The goal of this experiment is to illustrate the stability of our software when $\epsilon$ varies towards 0 . Recall from Section 6.1 that the $D$ operator is evaluated on a box in two different ways depending on how close to zero is the $t$-interval of that box. The Ball system is thus solved either with Equation (3.1) (involving a division by $\sqrt{t}$ ) when the values of the $t$-interval are larger than a parameter $\delta_{\text {Taylor }}\left(\right.$ set to $10^{-2}$ ) or using a Taylor expansion otherwise.

To illustrate the stability of our software, we compared in Table 2 its running times when $\epsilon$ varies towards 0 with what it would be without using Taylor expansions. It shows that if we do not use Taylor expansions, the solution is not certified (by the Ibexsolver; see Section 6.2 when $\epsilon \leqslant 10^{-5}$. On the other hand, our software is stable, although its running time increases from 0.1 to 0.8 seconds when $\epsilon$ gets smaller than or equal to $10^{-2}$, which is when the $D$ operator starts to be evaluated using a Taylor expansion. 


\begin{tabular}{|c|c|c|c|c|c|c|c|c|c|c|c|}
\hline$\epsilon$-values & $>1$ & 1 & 0.99 & 0.9 & $10^{-1}$ & $10^{-2}$ & $10^{-3}$ & $10^{-4}$ & $10^{-5}$ & $10^{-6}$ & $10^{-7}$ \\
\hline Time $\left(\delta_{\text {Taylor }}=10^{-2}\right)$ & \multirow{2}{*}{ 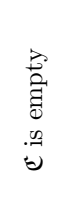 } & \multirow{2}{*}{ 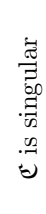 } & \multirow{2}{*}{\multicolumn{3}{|c|}{$\begin{array}{l}\text { Taylor forms are not } \\
\text { triggered }\end{array}$}} & \multicolumn{6}{|c|}{0.8} \\
\hline $\begin{array}{l}\text { Time without Taylor } \\
\text { forms }\end{array}$ & & & & & & \multicolumn{3}{|c|}{0.1} & \multicolumn{3}{|c|}{ Uncertified solutions } \\
\hline
\end{tabular}

Table 2: Experiment 4: Performances for different values of $\epsilon$.

\section{Genericity of the assumptions}

The key to prove the genericity of our assumptions is Thom's Transversality Theorem. We thus first recall, in Section 7.1 the basics of transversality theory using the notation of Demazure's book [Dem00]. We then prove, in Section 7.2, that all assumptions of Section 2 are satisfied for a generic curve. Finally, in Section 7.3, we consider the special case where the curve is the silhouette of a surface and prove that Assumptions $\mathcal{A}_{1}, \mathcal{A}_{2}, \mathcal{A}_{4}$ are generically satisfied in this case.

\subsection{Preliminaries}

We work with the set of smooth functions $C^{\infty}\left(\mathbb{R}^{n}, \mathbb{R}^{n-1}\right)$ with the weak (or compact-open) topology |Dem00 $\S 3.9 .2]$, that is convergence is understood as uniform on compact subsets and for any derivative. A subset of $C^{\infty}\left(\mathbb{R}^{n}, \mathbb{R}^{n-1}\right)$ is called residual if it contains the intersection of a countable family of dense open subsets. The space $C^{\infty}\left(\mathbb{R}^{n}, \mathbb{R}^{n-1}\right)$ is a Baire space [Dem00, Proposition 3.9.3], that is, every residual subset of $C^{\infty}\left(\mathbb{R}^{n}, \mathbb{R}^{n-1}\right)$ is dense. A property is generic in $C^{\infty}\left(\mathbb{R}^{n}, \mathbb{R}^{n-1}\right)$ if it is satisfied by a residual subset.

Definition 42 ([Dem00, \$3.8.3]). Let $E \simeq \mathbb{R}^{n}$ and $F$ be two finite-dimensional real vector spaces and let $r \geqslant 0$ be an integer. Let $P^{r}(E, F)$ be the vector space of polynomial functions of degree at most $r$ from $E$ to $F$. For an open subset $U$ of $E$ (with respect to the usual topology on $E$ ), let $J^{r}(U, F)=U \times P^{r}(E, F)$ be the space of jets of order $r$ of functions from $U$ to $F$. Notice that $J^{r}(U, F)$ can be identified with an open subset of $\mathbb{R}^{N}$ for some positive integer $N$. Let $f: U \rightarrow F$ be a smooth function, the jet of order $r$ of $f$ is the function

$$
\begin{aligned}
j^{r} f: U & \subset \mathbb{R}^{n} \rightarrow J^{r}(U, F) \subseteq \mathbb{R}^{N} \\
x & \mapsto\left(x, f(x), \frac{\partial f}{\partial x_{1}}(x), \ldots, \frac{\partial f}{\partial x_{n}}(x), \frac{\partial^{2} f}{\partial x_{1} \partial x_{2}}(x), \ldots, \frac{\partial^{r} f}{\partial x_{n}^{r}}(x)\right) .
\end{aligned}
$$

Let $W$ be a sub-manifold of $J^{r}(U, F)$. We say that $j^{r} f$ is transverse to $W$ if for all $a \in U$ either $j^{r} f(a) \notin W$ or every vector of $\mathbb{R}^{N}$ can be written as a sum of a vector of $T_{j^{r} f(a)} W$ and a vector in the image of the function $T_{a} j^{r} f$, where $T_{j^{r} f(a)} W$ is the tangent space of $W$ at $j^{r} f(a)$ and $T_{a} j^{r} f$ is the derivative function of $j^{r} f$ at $a$.

Theorem 43 (Thom's Transversality Theorem [Dem00, Theorem 3.9.4]). Let $E$ and $F$ be two finite-dimensional vector spaces with $U$ an open set in $E$. Let $r \geqslant 0$ be an integer and $W$ be a sub-manifold of $J^{r}(U, F)$. Then, the set of functions $f \in C^{\infty}(U, F)$ such that $j^{r} f$ is transverse to $W$ is a dense residual subset of $C^{\infty}(U, F)$. 
Proposition 44 ([Dem00, Corollary 3.7.3]). Let $U$ be an open subset of $\mathbb{R}^{n}, N \geqslant 1$ be an integer and $W$ be a sub-manifold of the vector space $\mathbb{R}^{N}$ of pure co-dimension $m$. Assume that the smooth function $g: U \rightarrow \mathbb{R}^{N}$ is transverse to $W$, then $g^{-1}(W)$ is a (possibly empty) sub-manifold of dimension $n-m$.

The idea of the proofs of genericity of our assumptions is to express each assumption as a system of equations in the jet space. When this system defines a manifold $W$, Proposition 44 directly applies to pull back the manifold from the jet space to the ambient space of the curve. This pull back is a sub-manifold of the same co-dimension as $W$. A difficulty occurs when the system does not define a manifold. The following corollary overcomes this difficulty in the special case where the system is defined by analytic functions, in other words, the system defines an analytic variety. Such a variety does not need to be a manifold, but, using the Whitney stratification theorem [Whi65], the variety is written as a union of manifolds on which Thom's theorem is then applied.

Corollary 45. Let $E$ and $F$ be two finite-dimensional vector spaces with $E$ of dimension $n$ and $U$ an open set in $E$. Let $r \geqslant 0$ be an integer and $W$ be an analytic variety of $J^{r}(U, F)$ with co-dimension larger than $n$, then for a generic $P \in C^{\infty}(U, F)$, the pre-image of $W$ under $j^{r} P$ is empty.

Proof. Let $W=\bigcup_{i=1}^{m} W_{i}$ be a Whitney stratification of $W$, where the $W_{i}$ 's are sub-manifolds. Since $\operatorname{codim}(W)>$ $n$, we have that $\operatorname{codim}\left(W_{i}\right)>n$ for any integer $1 \leqslant i \leqslant m$. Let $\Gamma_{i}=\left\{P \in C^{\infty}(U, F) \mid j^{r} P\right.$ is transverse to $\left.W_{i}\right\}$ and $\Gamma=\bigcap_{i=1}^{m} \Gamma_{i}$. By Theorem $43, \Gamma_{i}$ is residual and so is $\Gamma$. Moreover, by Proposition 44 , for $P \in \Gamma_{i}$ the pre-image of $W_{i}$ under $j^{r} P$ is empty. Hence, $\left(j^{r} P\right)^{-1}(W)=\bigcup_{i=1}^{m}\left(j^{r} P\right)^{-1}\left(W_{i}\right)=\emptyset$.

We will also need a refined version of Thom's theorem in a multijet setting, that is for several points in the source space simultaneously. We give the formal definitions of the multijet space and function but we do not restate versions of Theorem 43 . Proposition 44 and Corollary 45 that also hold for multijets.

Definition 46 ([Dem00, §3.9.6]). Let $U$ be an open subset of $\mathbb{R}^{n}$ and $k \geqslant 1$ be an integer. We denote $\Delta_{(k)}(U)$ the subset of $U^{k}$ consisting of sequences $\left(a_{1}, \ldots, a_{k}\right)$ of pairwise distinct points of $U$. For an integer $r \geqslant 0$ and a finite-dimensional space $F$, the k-multijet space of order $r, J_{(k)}^{r}(U, F)$, is the subset of $J^{r}(U, F)^{k}=$ $\left(U \times P^{r}(E, F)\right)^{k}$ consisting of the $k$-tuples $\left(\left(a_{1}, p_{1}\right), \ldots,\left(a_{k}, p_{k}\right)\right)$, with $\left(a_{1}, \ldots, a_{k}\right) \in \Delta_{(k)}(U)$. Let $f: U \rightarrow F$ be a smooth function, the $k$-multijet of order $r$ of $f$ is the function

$$
\begin{aligned}
j_{(k)}^{r} f: & \Delta_{(k)}(U) \rightarrow J_{(k)}^{r}(U, F) \\
& \left(a_{1}, \ldots, a_{k}\right) \mapsto\left(j^{r} f\left(a_{1}\right), \ldots, j^{r} f\left(a_{k}\right)\right) .
\end{aligned}
$$

Finally, we gather several technical tools from algebra and analysis.

Proposition 47 ([BV88, Proposition 1.A.1.1]). Let $M(m, n)$ be the vector space of real matrices of size $m \times n$ and $r$ be a positive integer such that $r<\min \{n, m\}$. The determinantal variety, $M_{r}$, is the set of matrices in $M(m, n)$ that have rank less than $r+1$. Then, the following statements hold: 
(a) $M_{r}$ is an irreducible variety in $M(m, n)$.

(b) $M_{r}$ is of dimension $r(n+m-r)$.

(c) The singular locus of $M_{r}$ is $M_{r-1}$.

Lemma 48 ([Bôc64, §XIV.61 Theorem 1]). Let $n \geqslant 2$ be an integer, $\left\{x_{i j}\right\}_{1 \leqslant j, i \leqslant n}$ be a set of $n^{2}$ variables and $\mathbb{C}\left[x_{i j}\right]_{1 \leqslant j, i \leqslant n}$ be the ring of complex polynomials with variables $\left\{x_{i j}\right\}$. Then, the determinant of the matrix $\left(x_{i j}\right)_{1 \leqslant i, j \leqslant n}$ is an irreducible polynomial in $\mathbb{C}\left[x_{i j}\right]_{1 \leqslant j, i \leqslant n}$.

Theorem 49 ([Whi43, Theorem $1 \& 2])$. Let $f$ be an even (resp. odd) smooth function, then there exists a smooth function $g$ such that $f(x)=g\left(x^{2}\right)\left(\right.$ resp. $\left.f(x)=x \cdot g\left(x^{2}\right)\right)$.

\subsection{Genericity of the assumptions for a curve in $\mathbb{R}^{n}$}

We are going to prove that each assumption in Section 2 is generic. Hence, the combination of these assumptions is also generic since a countable intersection of residual subsets in $C^{\infty}\left(\mathbb{R}^{n}, \mathbb{R}^{n-1}\right)$ is residual.

Lemma 50. Assumption $\widehat{\mathcal{A}_{1}}$ is generic.

Proof. Consider the jet of order 1 of the function $P \in C^{\infty}\left(\mathbb{R}^{n}, \mathbb{R}^{n-1}\right)$ :

$$
\begin{aligned}
j^{1} P: & \mathbb{R}^{n} \rightarrow J^{1}\left(\mathbb{R}^{n}, \mathbb{R}^{n-1}\right)=\mathbb{R}^{n} \times \mathbb{R}^{n-1} \times \mathbb{R}^{(n-1) \times n} \\
x & \mapsto\left(x, P(x), J_{P}(x)\right)=(x, y, z) .
\end{aligned}
$$

We represent the jet space by the variables $x \in \mathbb{R}^{n}, y \in \mathbb{R}^{n-1}$ and $z \in \mathbb{R}^{(n-1) \times n}$. Abusing notation, we can see the variable $z$ as an $(n-1) \times n$-matrix. Define the variety $W=\left\{(x, y, z) \in \mathbb{R}^{n} \times \mathbb{R}^{n-1} \times \mathbb{R}^{(n-1) \times n} \mid y=\right.$ $0, \operatorname{rank}(z) \leqslant n-2\}$. The variety $W$ is a product of a determinantal variety in $\mathbb{R}^{(n-1) \times n}$ of dimension $n^{2}-n-2$ (by Proposition 47) and a linear space of dimension $n$ in $\mathbb{R}^{n} \times \mathbb{R}^{n-1}$. Thus, $W$ is a variety of co-dimension $n+1$ in $\mathbb{R}^{n} \times \mathbb{R}^{n-1} \times \mathbb{R}^{(n-1) \times n}$. Hence, by Corollary 45 there exists a residual subset $\Gamma_{1} \subset C^{\infty}\left(\mathbb{R}^{n}, \mathbb{R}^{n-1}\right)$, such that for $P \in \Gamma_{1}$ the pre-image of $W$ under $j^{1} P$ is empty. Consequently, for a generic $P \in C^{\infty}\left(\mathbb{R}^{n}, \mathbb{R}^{n-1}\right)$ and any $q \in \overline{\mathfrak{C}}$, we have that $q \notin\left(j^{1} P\right)^{-1}(W)=\emptyset$, thus $\operatorname{rank}\left(J_{P}(q)\right)=n-1$, which is Assumption $\mathcal{A}_{1}$

Lemma 51. Assumption $\mathcal{A}_{2}$ is generic. Moreover, generically, the set $\overline{\mathfrak{L}_{\mathrm{c}}}$ is empty.

Proof. We consider the jet of order 1 of the function $P \in C^{\infty}\left(\mathbb{R}^{n}, \mathbb{R}^{n-1}\right)$ as in the proof of Lemma 50 with the same notation. Define the matrix $T_{1}(z)$ (resp. $T_{2}(z)$ ) to be the sub-matrix of $z$ obtained by removing the first (resp. second) column. Consider the variety $W \subset J^{1}\left(\mathbb{R}^{n}, \mathbb{R}^{n-1}\right)$ defined by $\left\{y=0 \in \mathbb{R}^{n-1}, \operatorname{det}\left(T_{1}(z)\right)=\right.$ $\left.\operatorname{det}\left(T_{2}(z)\right)=0\right\}$. Notice that $\overline{\mathfrak{L}_{\mathrm{c}}}$ is included in the pre-image of $W$ under $j^{1} P$ since $\overline{\mathfrak{L}_{\mathrm{c}}}$ is the set of points of the curve $\overline{\mathfrak{C}}$ that are both $x_{1}$ and $x_{2}$-critical. By Lemma 48 , we have that $\operatorname{both} \operatorname{det}\left(T_{1}(z)\right)$ and $\operatorname{det}\left(T_{2}(z)\right)$ are irreducible polynomials. By [CLO92, §9.4 Prop 10], a proper sub-variety of an irreducible variety is of lower dimension, we deduce that the common zero locus of $\operatorname{det}\left(T_{1}(z)\right)$ and $\operatorname{det}\left(T_{2}(z)\right)$ is of co-dimension at least two. 
We deduce that $\operatorname{codim}(W)>n$. By Corollary 45 , there exists a residual subset $\Gamma_{2} \subset C^{\infty}\left(\mathbb{R}^{n}, \mathbb{R}^{n-1}\right)$, such that for $P \in \Gamma_{2} \cap \Gamma_{1}$, the pre-image of $W$ under $j^{1} P$ is empty and hence $\overline{\mathfrak{L}_{\mathrm{c}}}$ is empty, which implies Assumption $\mathcal{A}_{2}$

Lemma 52. Assumption $\mathcal{A}_{3}$ is generic.

Proof. Let us consider the 3-multijet of order 0:

$$
\begin{aligned}
j_{(3)}^{0} P: \Delta_{(3)}\left(\mathbb{R}^{n}\right) & \rightarrow J_{(3)}^{0}\left(\mathbb{R}^{n}, \mathbb{R}^{n-1}\right)=\left(\mathbb{R}^{n} \times \mathbb{R}^{n-1}\right)^{3} \\
\left(x, x^{\prime}, x^{\prime \prime}\right) & \mapsto\left((x, P(x)),\left(x^{\prime}, P\left(x^{\prime}\right)\right),\left(x^{\prime \prime}, P\left(x^{\prime \prime}\right)\right)\right)=\left((x, y),\left(x^{\prime}, y^{\prime}\right),\left(x^{\prime \prime}, y^{\prime \prime}\right)\right)
\end{aligned}
$$

where every element in the jet space $J_{(3)}^{0}\left(\mathbb{R}^{n}, \mathbb{R}^{n-1}\right)$ is of the form $\left((x, y),\left(x^{\prime}, y^{\prime}\right),\left(x^{\prime \prime}, y^{\prime \prime}\right)\right)$, where $x=$ $\left(x_{1}, \ldots, x_{n}\right), x^{\prime}, x^{\prime \prime} \in \mathbb{R}^{n}$ and $y, y^{\prime}, y^{\prime \prime} \in \mathbb{R}^{n-1}$. Consider the linear sub-manifold $W=\left\{x_{1}=x_{1}^{\prime}=x_{1}^{\prime \prime}, x_{2}=\right.$ $\left.x_{2}^{\prime}=x_{2}^{\prime \prime}, y=y^{\prime}=y^{\prime \prime}=0\right\}$, the co-dimension of $W$ is thus $3 n+1$ which is larger than the dimension of the source space $\Delta_{(3)}\left(\mathbb{R}^{n}\right)$ which is $3 n$. Thus, by Corollary 45 there exists a residual subset $\Gamma_{3} \subset C^{\infty}\left(\mathbb{R}^{n}, \mathbb{R}^{n-1}\right)$, such that for $P \in \Gamma_{3}$, the pre-image of $W$ by $j_{(3)}^{0}$ is empty, which translates to the fact that there are no pairwise distinct points $q, q^{\prime}, q^{\prime \prime}$ in $\overline{\mathfrak{C}}$ such that $\pi_{\overline{\mathfrak{C}}}(q)=\pi_{\overline{\mathfrak{C}}}\left(q^{\prime}\right)=\pi_{\overline{\mathfrak{C}}}\left(q^{\prime \prime}\right)$. This is also equivalent to saying that the system $S=\left\{P(x)=0 \in \mathbb{R}^{n-1}, x_{1}-\alpha=x_{2}-\beta=0\right\}$ has at most two distinct solutions (without counting multiplicities) for any $(\alpha, \beta) \in \mathbb{R}^{2}$.

Using $\Gamma_{1}, \Gamma_{2}$ as defined in the proofs of Lemmas 50 \&1] and $\Gamma_{3}$ defined above, we define $\Gamma_{4}=\Gamma_{1} \cap \Gamma_{2} \cap \Gamma_{3}$ which is thus a residual set and let $P$ be in $\Gamma_{4}$. Since $P$ is in $\Gamma_{3}$, the system $S$ has at most two distinct solutions. In addition, since $P$ is in $\Gamma_{2} \cap \Gamma_{1}$, one has that $\overline{\mathfrak{L}_{\mathrm{c}}}$ is empty and finally together with Lemma 20 , since $P$ is in $\Gamma_{1}$, this implies that these solutions have multiplicity exactly 1 in $S$. For $P$ in the residual set $\Gamma_{4}$, the number of solutions counted with multiplicities of $S$ is thus at most 2, which is Assumption $\mathcal{A}_{3}$

Lemma 53. Assumption $\mathcal{A}_{4}$ is generic.

Proof. Let us consider the 2-multijet of order 0 of $P$ :

$$
\begin{aligned}
j_{(2)}^{0} P: & \Delta_{(2)}\left(\mathbb{R}^{n}\right) \rightarrow J_{(2)}^{0}\left(\mathbb{R}^{n}, \mathbb{R}^{n-1}\right)=\left(\mathbb{R}^{n} \times \mathbb{R}^{n-1}\right)^{2} \\
\left(x, x^{\prime}\right) & \mapsto\left((x, P(x)),\left(x^{\prime}, P\left(x^{\prime}\right)\right)\right)=\left((x, y),\left(x^{\prime}, y^{\prime}\right)\right)
\end{aligned}
$$

where every element in the jet space $J_{(2)}^{0}\left(\mathbb{R}^{n}, \mathbb{R}^{n-1}\right)$ is of the form $\left((x, y),\left(x^{\prime}, y^{\prime}\right)\right)$, where $x=\left(x_{1}, \ldots, x_{n}\right)$, $x^{\prime} \in \mathbb{R}^{n}$ and $y, y^{\prime} \in \mathbb{R}^{n-1}$. Consider the linear sub-manifold $W=\left\{x_{1}=x_{1}^{\prime}, x_{2}=x_{2}^{\prime}, y=y^{\prime}=0\right\}$ of the jet space $J_{(2)}^{0}\left(\mathbb{R}^{n}, \mathbb{R}^{n-1}\right)$. Notice that, $\left(j_{(2)}^{0} P\right)^{-1}(W)$ contains the set $\widehat{\mathfrak{L}}_{\mathrm{n}}^{\prime}=\left\{\left(q_{1}, q_{2}\right) \in \Delta_{(2)}\left(\mathbb{R}^{n}\right) \cap \overline{\mathfrak{C}} \times \overline{\mathfrak{C}} \mid \pi_{\overline{\mathfrak{C}}}\left(q_{1}\right)=\right.$ $\left.\pi_{\overline{\mathfrak{C}}}\left(q_{2}\right)\right\}$ and $\overline{\mathfrak{L}_{\mathrm{n}}}$ is the image of $\widehat{\mathfrak{L}}_{\mathrm{n}}^{\prime}$ by the projection $\left(q_{1}, q_{2}\right) \rightarrow q_{1}$. We have $\operatorname{dim}\left(\Delta_{(2)}\left(\mathbb{R}^{n}\right)\right)=2 n$ and, since $W$ is linear, its co-dimension is easily computed $\operatorname{codim}(W)=2(2 n-1)-(2+2(n-1))=2 n$. Proposition 44 thus yields that generically $\left(j_{(2)}^{0} P\right)^{-1}(W)$ is a sub-manifold of dimension zero that is a discrete set in $\mathbb{R}^{n}$, and so is $\overline{\mathfrak{L}_{n}}$. 
Now, we prove that, generically, $\overline{\mathfrak{L}_{\mathrm{n}}}$ does not intersect the boundary of $B$. The boundary $\partial B$ of the box $B$ is included in the union of the supporting hyperplanes $H_{i}$ of its $2^{n}$ faces of dimension $n-1$, that is $\partial B=\cup_{i=1}^{i=2^{n}} H_{i}$. Define the linear sub-manifold $W_{i}=\left\{\left((x, y),\left(x^{\prime}, y^{\prime}\right)\right) \in W \mid x \in H_{i}\right.$ or $\left.x^{\prime} \in H_{i}\right\}$, notice that this adds one equation to $W$ and thus increases the co-dimension of $W$ by one, thus $\operatorname{codim}\left(W_{i}\right)=2 n+1$. By Corollary 45 , we have that generically, the pre-image of $W_{i}$ under $j_{(2)}^{0} P$ is empty, which translates to the fact that there is no point of $\overline{\mathfrak{L}_{\mathrm{n}}}$ on $\partial B \cap H_{i}$. This is also true for any $i$ and thus, generically, $\overline{\mathfrak{L}_{\mathrm{n}}}$ does not intersect the boundary of $B$.

Corollary 54. Assumption $\mathcal{A}_{5}$ is generic.

Proof. Let $B$ be an open $n$-box. We prove that for a generic $P \in C^{\infty}\left(\mathbb{R}^{n}, \mathbb{R}^{n-1}\right)$, the singular points of $\pi_{\mathfrak{C}}(\mathfrak{C})$ are only nodes (recall that by Lemma 26 , under the generic assumptions $\mathcal{A}_{1}, \mathcal{A}_{2} \mid \mathcal{A}_{3}$, and $\mathcal{A}_{4}$, the points in $\mathfrak{C} \backslash\left(\mathfrak{L}_{\mathrm{c}} \cup \mathfrak{L}_{\mathrm{n}}\right)$ project to smooth points).

Let $\Gamma_{0}$ be the set of $P \in C^{\infty}\left(\mathbb{R}^{n}, \mathbb{R}^{n-1}\right)$ such that $P$ satisfies Assumptions $\mathcal{A}_{1}, \mathcal{A}_{2}, \mathcal{A}_{3}$ and $\mathcal{A}_{4}$. The previous lemmas of this section show that $\Gamma_{0}$ is residual in $C^{\infty}\left(\mathbb{R}^{n}, \mathbb{R}^{n-1}\right)$. Let us consider the 2-multijet of order 1 of $P$ :

$$
\begin{aligned}
j_{(2)}^{1} P: \Delta_{(2)}\left(\mathbb{R}^{n}\right) \rightarrow J_{(2)}^{1}\left(\mathbb{R}^{n}, \mathbb{R}^{n-1}\right) \subseteq\left(\mathbb{R}^{n} \times \mathbb{R}^{n-1} \times \mathbb{R}^{(n-1) \times n}\right)^{2} \\
\left(x, x^{\prime}\right) \mapsto\left(\left(x, P(x), J_{P}(x)\right),\left(x^{\prime}, P\left(x^{\prime}\right), J_{P}\left(x^{\prime}\right)\right)\right)=\left((x, y, z),\left(x^{\prime}, y^{\prime}, z^{\prime}\right)\right)
\end{aligned}
$$

Let $s, s^{\prime}$ (resp. $r, r^{\prime}$ ) be the sub-matrices of $z, z^{\prime}$, respectively, obtained by removing the first two columns (resp. obtained by the first two columns). Define the matrix $M=\left(\begin{array}{ccc}r & 0 & s \\ r^{\prime} & s^{\prime} & 0\end{array}\right)$ and the variety

$$
W=\left\{\left((x, y, z),\left(x^{\prime}, y^{\prime}, z^{\prime}\right)\right) \in\left(\mathbb{R}^{n} \times \mathbb{R}^{n-1} \times \mathbb{R}^{(n-1) \times n}\right)^{2} \mid y=y^{\prime}=0, x_{1}=x_{1}^{\prime}, x_{2}=x_{2}^{\prime}, \operatorname{det}(M)=0\right\} .
$$

The variety $W$ is a product of a determinantal variety and a linear space, thus its co-dimension is $\operatorname{codim}(W) \geqslant$ $2 n+1>2 n=\operatorname{dim}\left(\Delta_{(2)}\left(\mathbb{R}^{n}\right)\right)$. Hence, by Corollary 45 , there exists a residual subset $\Gamma_{0}^{\prime}$ in $C^{\infty}\left(\mathbb{R}^{n}, \mathbb{R}^{n-1}\right)$ such that for all $P \in \Gamma_{0}^{\prime}$, the pre-image of $W$ under $j_{(2)}^{1} P$ is empty.

Let $P$ be in the residual set $\Gamma_{0} \cap \Gamma_{0}^{\prime}$. By Lemma 31 and since $\mathfrak{L}_{\mathrm{c}}$ is empty, we deduce that for distinct $q_{1}, q_{2} \in \mathfrak{C}$ with $\pi_{\mathfrak{C}}\left(q_{1}\right)=\pi_{\mathfrak{C}}\left(q_{2}\right)$, the plane projections of the lines $T_{q_{1}} \mathfrak{C}$ and $T_{q_{2}} \mathfrak{C}$ intersect transversely if and only if $j_{(2)}^{1}\left(\left(q_{1}, q_{2}\right)\right) \notin W$. Finally, by Lemma 21 (Step (a) of the proof), we deduce that $\pi_{\mathfrak{C}}\left(q_{1}\right)=\pi_{\mathfrak{C}}\left(q_{2}\right)$ is a node in $\pi_{\mathfrak{C}}(\mathfrak{C})$.

\subsection{Genericity of the assumptions for the silhouette of a surface in $\mathbb{R}^{n}$}

In this section, we focus on the special case of silhouette curves of surfaces in $\mathbb{R}^{n}$. For an open $n$-box $B$ and $\widetilde{P}$ in $C^{\infty}\left(\mathbb{R}^{n}, \mathbb{R}^{n-2}\right)$ such that $S=\widetilde{P}^{-1}(0)$ is a smooth 2-sub-manifold in $\mathbb{R}^{n}$, the silhouette of $\widetilde{P}$ is the set of points $q$ of this surface $S$ such that the projection (with respect to a fixed direction) of the tangent plane $T_{q} S$ to $\mathbb{R}^{2}$ is not surjective. We prove that Assumptions $\mathcal{A}_{1}, \mathcal{A}_{2} \& \mathcal{A}_{4}$ are satisfied for a generic silhouette, and we only conjecture that Assumptions $\mathcal{A}_{3} \& \mathcal{A}_{5}^{-}$also hold generically. We start by formalizing the definition of the silhouette curve algebraically. 
Definition 55. For an integer $n \geqslant 3$, let $\widetilde{P}=\left(P_{1}, \ldots, P_{n-2}\right) \in C^{\infty}\left(\mathbb{R}^{n}, \mathbb{R}^{n-2}\right)$. Define the smooth function $P_{n-1}=\operatorname{det}\left(\left(\frac{\partial P_{i}}{\partial x_{j}}\right)_{\substack{1 \leqslant i \leqslant n-2 \\ 3 \leqslant j \leqslant n}}\right)$ and $P=\left(P_{1}, \ldots, P_{n-1}\right)$. We define the curve $\mathfrak{C}$ (and $\left.\overline{\mathfrak{C}}\right)$ as in Section 2 and call it the silhouette of $\widetilde{P}$.

Proposition 56. For a generic $\widetilde{P} \in C^{\infty}\left(\mathbb{R}^{n}, \mathbb{R}^{n-2}\right)$, the function P satisfies Assumption $\mathcal{A}_{1}$

Proof. Consider the jet of order 1 of $\widetilde{P}$ :

$$
\begin{aligned}
j^{1} \widetilde{P}: & \mathbb{R}^{n} \rightarrow J^{1}\left(\mathbb{R}^{n}, \mathbb{R}^{n-2}\right)=\mathbb{R}^{n} \times \mathbb{R}^{n-2} \times \mathbb{R}^{(n-2) \times n} \simeq \mathbb{R}^{n^{2}-2}=\mathbb{R}^{N} \\
x & \mapsto\left(x, \widetilde{P}(x), J_{\widetilde{P}}(x)\right)=(x, y, z) .
\end{aligned}
$$

We represent the jet space by the vectors $x \in \mathbb{R}^{n}, y \in \mathbb{R}^{n-2}$ and the $((n-2) \times n)$-matrix $z \in \mathbb{R}^{(n-2) \times n}$. Let $T(z)$ denote the sub-matrix obtained by removing the first two columns of $z$. Define the variety $W=\{y=$ $0, \operatorname{det}(T(z))=0\}=\{y=0, \operatorname{rank}(T(z)) \leqslant n-3\}$ in $\mathbb{R}^{N}$. According to Proposition 47, $W=\operatorname{Reg}(W) \cup$ $\operatorname{Sing}(W)$ where $\operatorname{Reg}(W)$ (resp. Sing $(W)$ ) is the set of smooth (resp. singular) points in $W$ and

$$
\begin{gathered}
\operatorname{Reg}(W)=\left\{(x, y, z) \in \mathbb{R}^{N} \mid y=0, \operatorname{rank}(T(z))=n-3\right\} \\
\operatorname{Sing}(W)=\left\{(x, y, z) \in \mathbb{R}^{N} \mid y=0, \operatorname{rank}(T(z))<n-3\right\} .
\end{gathered}
$$

In addition, Proposition 47 yields that $\operatorname{Reg}(W)$ is a manifold of co-dimension $n-1$ and $\operatorname{Sing}(W)$ is a variety of co-dimension $n+2$. Since the co-dimension of $\operatorname{Sing}(W)$ is larger than that of the source space, Corollary 45 implies that, generically, $\left(j^{1} \widetilde{P}\right)^{-1}(\operatorname{Sing}(W))=\emptyset$. One thus has $\left(j^{1} \widetilde{P}\right)^{-1}(W)=\left(j^{1} \widetilde{P}\right)^{-1}(\operatorname{Reg}(W))$.

Consider the function

$$
\begin{gathered}
\varphi: \mathbb{R}^{n} \times \mathbb{R}^{n-2} \times \mathbb{R}^{(n-2) \times n} \rightarrow \mathbb{R}^{n-2} \times \mathbb{R} \\
\chi=(x, y, z) \mapsto(y, \operatorname{det}(T(z))),
\end{gathered}
$$

such that $\varphi^{-1}(0)=W$. Its Jacobian matrix is $J_{\varphi}=\left(\begin{array}{ccc}0_{(n-2) \times n} & I_{(n-2) \times(n-2)} & 0_{(n-2) \times(n-2) n} \\ 0_{1 \times(n)} & 0_{1 \times(n-2)} & v(z)\end{array}\right)$, where $0_{k_{1} \times k_{2}}$ (resp. $I_{k_{1} \times k_{2}}$ ) is the zero (resp. identity) matrix of size $k_{1} \times k_{2}$ and the vector $v(z)$ is the adjugate matrix of $T(z)$ written as the concatenation of its lines: $v(z)=\left(A d j^{i j}(T(z))\right)_{\substack{1 \leqslant i \leqslant n-2 \\ 3 \leqslant j \leqslant n}} \in \mathbb{R}^{(n-2)^{2}}$. Let $\chi=(x, y, z) \in \operatorname{Reg}(W)$, then $\operatorname{rank}(T(z))=n-3$, thus there exists a pair $(i, j)$ such that $A d j^{i j}(T(z)) \neq 0$. Hence, the vector $v(z)$ is non-trivial and $J_{\varphi}(\chi)$ has full rank $n-1$. The function $\varphi$ is thus a submersion on $\operatorname{Reg}(W)$.

Theorem 43 yields that, generically, $j^{1} \widetilde{P}$ is transverse to the manifold $\operatorname{Reg}(W)$. Together with the fact that $\varphi$ is a submersion on $\operatorname{Reg}(W)$, GG73, Lemma II.4.3 (p.52)] implies that $P=\varphi \circ j^{1} \widetilde{P}$ is a submersion on $\left(j^{1} \widetilde{P}\right)^{-1}(\operatorname{Reg}(W))=\left(j^{1} \widetilde{P}\right)^{-1}(W)=\left(j^{1} \widetilde{P}\right)^{-1}\left(\varphi^{-1}(0)\right)=\left(\varphi \circ j^{1} \widetilde{P}\right)^{-1}(0)=P^{-1}(0)=\mathfrak{C}$. In other words, $J_{P}$ has full rank $n-1$ on $\mathfrak{C}$, which is Assumption $\mathcal{A}_{1}$.

Proposition 57. For a generic $\widetilde{P} \in C^{\infty}\left(\mathbb{R}^{n}, \mathbb{R}^{n-2}\right)$, the function P satisfies Assumption $\mathcal{A}_{2}$ 
Proof. First we prove that, generically, $\overline{\mathfrak{L}_{\mathrm{c}}}$ is discrete. For any $\widetilde{P} \in C^{\infty}\left(\mathbb{R}^{n}, \mathbb{R}^{n-2}\right)$ consider $j^{2} \widetilde{P}: \mathbb{R}^{n} \rightarrow$ $J^{2}\left(\mathbb{R}^{n}, \mathbb{R}^{n-2}\right) \subset \mathbb{R}^{n} \times \mathbb{R}^{n-2} \times \mathbb{R}^{(n-2) \times n} \times \mathbb{R}^{n^{2}(n-2)}=\mathbb{R}^{N}$. Assume that every element in $\mathbb{R}^{N}$ is represented as $(x, y, z, h)$, where $x \in \mathbb{R}^{n}, y \in \mathbb{R}^{n-2}, z \in \mathbb{R}^{(n-2) \times n}$ and $h \in \mathbb{R}^{n^{2}(n-2)}$. Abusing notation, we consider $z$ as a $((n-2) \times n)$-matrix. Let $T(z)$ denote the matrix obtained by removing the first two columns of $z$. The Jacobian matrix $J_{P}$ is a function of the derivatives $\left(\frac{\partial P_{i}}{\partial x_{j}}, \frac{\partial^{2} P_{l}}{\partial x_{k} \partial x_{s}}\right)_{\substack{1 \leqslant i, l \leqslant n-2 \\ 1 \leqslant j, k, s \leqslant n}}$, it can thus be seen in the jet space as a function of $z$ and $h, J_{P}(z, h)$. Define the matrix $T_{1}(z, h)$ (resp. $T_{2}(z, h)$ ) to be the sub-matrix of $J_{P}(z, h)$ obtained by removing the first (resp. second) column. Define the variety $W=\{(x, y, z, h) \mid y=0 \in$ $\left.\mathbb{R}^{n-2}, \operatorname{det}(T(z))=\operatorname{det}\left(T_{1}(z, h)\right)=\operatorname{det}\left(T_{2}(z, h)\right)=0\right\}$, so that $\overline{\mathfrak{L}_{\mathrm{c}}}$ is included in the pre-image of $W$ under $j^{2} \widetilde{P}$. Let $W_{1}=\left\{(x, y, z, h) \mid y=0 \in \mathbb{R}^{n-2}, \operatorname{det}(T(z))=0\right\}$, we already showed in the proof of Proposition 56 that $W_{1}$ is an irreducible variety of co-dimension $n-1$. In addition, $\operatorname{det}\left(T_{1}(z, h)\right)$ does not identically vanish on $W_{1}$, thus $W$ is a proper sub-variety of the irreducible variety $W_{1}$ and [CLO92, §9.4 Prop 10] implies that $\operatorname{codim}(W)>\operatorname{codim}\left(W_{1}\right)=n-1$.

Now, write $W=\operatorname{Reg}(W) \cup \operatorname{Sing}(W)$, where $\operatorname{Reg}(W)$ (resp. $\operatorname{Sing}(W)$ ) is the set of smooth (resp. singular) points in $W$. Recall that $\operatorname{codim}(\operatorname{Sing}(W))>n$ since $\operatorname{Sing}(W)$ is a proper closed sub-variety of $W$ [BCR98, Proposition 3.3.14]. By Corollary 45, there exists a residual set $\Gamma^{\prime} \subset C^{\infty}\left(\mathbb{R}^{n}, \mathbb{R}^{n-2}\right)$ such that if $\widetilde{P} \in \Gamma^{\prime}$, then the pre-image of $\operatorname{Sing}(W)$ under $j^{2} \widetilde{P}$ is empty. Define $\Gamma=\left\{\widetilde{P} \in C^{\infty}\left(\mathbb{R}^{n}, \mathbb{R}^{n-2}\right)\right.$ $j^{2} \widetilde{P}$ is transverse to $\left.\operatorname{Reg}(W)\right\} \cap \Gamma^{\prime}$. Notice that if $\widetilde{P} \in \Gamma$, then $\overline{\mathfrak{L}_{\mathrm{c}}}$ is included in the pre-image of $\operatorname{Reg}(W)$ under $j^{2} \widetilde{P}$. Hence, since $\operatorname{codim}(\operatorname{Reg}(W))=\operatorname{codim}(W) \geqslant n$, we have by Proposition 44 that $\overline{\mathfrak{L}_{\mathrm{c}}}$ is a sub-manifold of dimension, at most, zero. Thus, $\overline{\mathfrak{L}_{\mathrm{c}}}$ is discrete for all $\widetilde{P} \in \Gamma$. Using Theorem 43 we deduce that $\Gamma$ is residual.

The proof that $\overline{\mathfrak{L}_{\mathrm{c}}}$ does not intersect the boundary of $B$ can be done analogously as in the proof of Lemma 53

Proposition 58. For a generic $\widetilde{P} \in C^{\infty}\left(\mathbb{R}^{n}, \mathbb{R}^{n-2}\right)$, the function P satisfies Assumption $\mathcal{A}_{4}$

Proof. Consider the 2-multijet $j_{(2)}^{1} \widetilde{P}: \Delta_{(2)}\left(\mathbb{R}^{n}\right) \rightarrow J_{(2)}^{1}\left(\mathbb{R}^{n}, \mathbb{R}^{n-2}\right)=\left(\mathbb{R}^{n} \times \mathbb{R}^{n-2} \times \mathbb{R}^{(n-2) \times n}\right)^{2}$ of the function $\widetilde{P} \in C^{\infty}\left(\mathbb{R}^{n}, \mathbb{R}^{n-2}\right)$, where $\left(\mathbb{R}^{n} \times \mathbb{R}^{(n-2) \times n} \times \mathbb{R}^{(n-2) \times n}\right)^{2}$ is described by the coordinates $x, x^{\prime} \in \mathbb{R}^{n}, y, y^{\prime} \in$ $\mathbb{R}^{n-2}$ and $z, z^{\prime} \in \mathbb{R}^{(n-2) \times n}$. Abusing notation, we consider $z$ and $z^{\prime}$ as $((n-2) \times n)$ - matrices. Let $T(z)$ (resp. $T\left(z^{\prime}\right)$ ) denote the matrix obtained by removing the first two columns of $z$ (resp. $z^{\prime}$ ). Define the variety $W$ to be the solution set of the system $\left\{y=y^{\prime}=0, x_{1}-x_{1}^{\prime}=x_{2}-x_{2}^{\prime}=\operatorname{det}(T(z))=\operatorname{det}\left(T\left(z^{\prime}\right)\right)=0\right\}$. Denote the regular part of $W$ by $\operatorname{Reg}(W)$. By Proposition 47 (a) we deduce that $W$ is of co-dimension $2 n$. Using the same argument in the proof of Proposition 56, we deduce that there exists a residual set $\Gamma \subset C^{\infty}\left(\mathbb{R}^{n}, \mathbb{R}^{n-2}\right)$ such that if $\widetilde{P} \in \Gamma$, then the image of $\Delta_{2}\left(\mathbb{R}^{n}\right)$ under $j_{(2)}^{1} \widetilde{P}$ is contained in $\operatorname{Reg}(W)$. Moreover, by Proposition 44, we have that $M_{P}=\left(j_{(2)}^{1} \widetilde{P}\right)^{-1}(\operatorname{Reg}(W))=\left(j_{(2)}^{1} \widetilde{P}\right)^{-1}(W)$ is a sub-manifold of dimension zero in $\Delta_{2}\left(\mathbb{R}^{n}\right)$. Notice that $\overline{\mathfrak{L}_{\mathrm{n}}}$ is the image of $M_{P}$ under the projection $\left(x, x^{\prime}\right) \rightarrow x$. Since $M_{P}$ is of dimension zero, then so is $\overline{\mathfrak{L}_{\mathrm{n}}}$. Thus we have just proven that, if $\widetilde{P} \in \Gamma$, then $\overline{\mathfrak{L}_{\mathrm{n}}}$ is a sub-manifold of dimension zero. Hence, $\overline{\mathfrak{L}_{\mathrm{n}}}$ is discrete.

The proof that $\overline{\mathfrak{L}_{\mathrm{n}}}$ does not intersect the boundary of $B$ can be done analogously as in the proof of Lemma 53 
Assumption $\mathcal{A}_{3}$ can be rephrased by the three following assumptions:

$\mathcal{A}_{3(a)}$ - There are no pairwise distinct $q, q^{\prime}, q^{\prime \prime} \in \overline{\mathfrak{C}}$ such that $\pi_{\mathfrak{C}}(q)=\pi_{\mathfrak{C}}\left(q^{\prime}\right)=\pi_{\mathfrak{C}}\left(q^{\prime \prime}\right)$.

$\mathcal{A}_{3(b)}-\overline{\mathfrak{L}_{\mathrm{c}}} \cap \overline{\mathfrak{L}_{\mathrm{n}}}=\emptyset$.

$\mathcal{A}_{3(c)}$ - For $q \in \overline{\mathfrak{L}_{\mathrm{c}}}$, the multiplicity of the system $\left\{P(x)=0 \in \mathbb{R}^{n-1},\left(x_{1}, x_{2}\right)=\pi_{\mathfrak{C}}(q)\right\}$ at $q$ is exactly two.

Using this rephrasing, we next show that Assumptions $\mathcal{A}_{3(a)} \& \mathcal{A}_{3(b)}$ generically hold and we leave the genericity of Assumption $\overline{\mathcal{A}_{3(c)}}$ as a conjecture.

Proposition 59. For a generic function $\widetilde{P} \in C^{\infty}\left(\mathbb{R}^{n}, \mathbb{R}^{n-2}\right)$, Assumption $\overline{\mathcal{A}_{3(a)}}$ holds.

Proof. Consider the 3-multijet $j_{(3)}^{1} \widetilde{P}: \Delta_{(3)}\left(\mathbb{R}^{n}\right) \rightarrow J_{(3)}^{1}\left(\mathbb{R}^{n}, \mathbb{R}^{n-2}\right)=\left(\mathbb{R}^{n} \times \mathbb{R}^{n-2} \times \mathbb{R}^{(n-2) \times n}\right)^{3}$. Assume that every element in $\left(\mathbb{R}^{n} \times \mathbb{R}^{n-2} \times \mathbb{R}^{(n-2) n}\right)^{3}$ is of the form $\left((x, y, z),\left(x^{\prime}, y^{\prime}, z^{\prime}\right),\left(x^{\prime \prime}, y^{\prime \prime}, z^{\prime \prime}\right)\right)$, where $x, x^{\prime}, x^{\prime \prime} \in \mathbb{R}^{n}$, $y, y^{\prime}, y^{\prime \prime} \in \mathbb{R}^{n-2}$ and $z, z^{\prime}, z^{\prime \prime} \in \mathbb{R}^{(n-2) \times n}$. Abusing notation, we consider $z, z^{\prime}$ and $z^{\prime \prime}$ as $((n-2) \times n)$-matrices. Let $T(z), T\left(z^{\prime}\right), T\left(z^{\prime \prime}\right)$ denote the matrices obtained by removing the first two columns of $z, z^{\prime}, z^{\prime \prime}$, respectively. Consider the variety $W$ defined by the equations: $\left\{x_{1}=x_{1}^{\prime}=x_{1}^{\prime \prime}, x_{2}=x_{2}^{\prime}=x_{2}^{\prime \prime}, y=y^{\prime}=y^{\prime \prime}=0 \in\right.$ $\left.\mathbb{R}^{n-2}, \operatorname{det}(T(z))=\operatorname{det}\left(T\left(z^{\prime}\right)\right)=\operatorname{det}\left(T\left(z^{\prime \prime}\right)\right)=0\right\}$.

Notice that $\operatorname{dim}\left(\Delta_{(3)}\left(\mathbb{R}^{n}\right)\right)=3 n<3 n+1=\operatorname{codim}(W)$. Hence, by Corollary 45 , we have that, generically, the pre-image of $W$ under $j_{(3)}^{1} \widetilde{P}$ is empty. Hence, there are no pairwise different $q, q^{\prime}, q^{\prime \prime} \in \overline{\mathfrak{C}}$ such that $\pi_{\mathfrak{C}}(q)=$ $\pi_{\mathfrak{C}}\left(q^{\prime}\right)=\pi_{\mathfrak{C}}\left(q^{\prime \prime}\right)$

Proposition 60. For a generic function $\widetilde{P} \in C^{\infty}\left(\mathbb{R}^{n}, \mathbb{R}^{n-2}\right)$, Assumption $\mathcal{A}_{3(b)}$ holds.

Proof. Consider the 2-multijet $j_{(2)}^{2} \widetilde{P}: \Delta_{(2)}\left(\mathbb{R}^{n}\right) \rightarrow J_{(2)}^{2}\left(\mathbb{R}^{n}, \mathbb{R}^{n-2}\right)=\left(\mathbb{R}^{n} \times \mathbb{R}^{n-2} \times \mathbb{R}^{(n-2) \times n} \times \mathbb{R}^{n^{2}(n-2)}\right)^{2}$ of the function $\widetilde{P} \in C^{\infty}\left(\mathbb{R}^{n}, \mathbb{R}^{n-2}\right)$, where $\left(\mathbb{R}^{n} \times \mathbb{R}^{(n-2) \times n} \times \mathbb{R}^{(n-2) \times n} \times \mathbb{R}^{n^{2}(n-2)}\right)^{2}$ is described by the coordinates $x, x^{\prime} \in \mathbb{R}^{n}, y, y^{\prime} \in \mathbb{R}^{n-2}, z, z^{\prime} \in \mathbb{R}^{(n-2) \times n}$ and $h, h^{\prime} \in \mathbb{R}^{n^{2}(n-2)}$. With abuse of notation we can consider $z$ and $z^{\prime}$ as $((n-2) \times n)$ - matrices. Let $T(z)$ (resp. $\left.T\left(z^{\prime}\right)\right)$ denote the matrix obtained by removing the first two columns of $z$ (resp. $z^{\prime}$ ). Define the matrices $T_{1}(z, h), T_{2}(z, h)$ as in the proof of Lemma 57 and the variety $W$ to be the solution set of the system $\left\{y=y^{\prime}=0 \in \mathbb{R}^{n-2}, x_{1}-x_{1}^{\prime}=x_{2}-x_{2}^{\prime}=\operatorname{det}(T(z))=\operatorname{det}\left(T\left(z^{\prime}\right)=0\right.\right.$, $\left.\operatorname{det}\left(T_{1}(z, h)\right)=\operatorname{det}\left(T_{2}(z, h)\right)=0\right\}$.

Define varieties $W^{\prime}=\left\{(x, y, z, h) \mid y=y^{\prime}=0, \operatorname{det}(T(z))=\operatorname{det}\left(T\left(z^{\prime}\right)\right)=0, x_{1}=x_{1}^{\prime}, x_{2}=x_{2}^{\prime}\right\}$ and $W^{\prime \prime}=\left\{(x, y, z, h) \mid y=y^{\prime}=0, \operatorname{det}\left(T_{1}(z, h)\right)=\operatorname{det}\left(T_{2}(z, h)\right)=0\right\}$. Notice that $W=W^{\prime} \cap W^{\prime \prime}$. Moreover, we can find a smooth silhouette curve $C$ that is not an orthogonal line to the $\left(x_{1}, x_{2}\right)$-plane and that contains two distinct points $q, q^{\prime}$, with $\pi_{\mathfrak{C}}(q)=\pi_{\mathfrak{C}}\left(q^{\prime}\right)$ such that the projection of $T_{q} C$ (resp. $T_{q^{\prime}} C$ ) onto $\mathbb{R}^{2}$ is injective. Notice that $j_{(2)}^{2} \widetilde{P}\left(q, q^{\prime}\right) \in W^{\prime} \backslash W^{\prime \prime}$. Hence, $W^{\prime} \nsubseteq W^{\prime \prime}$. Moreover, since $W^{\prime}$ is the Cartesian product of determinant varieties (which are irreducible by Proposition 47 a) with linear spaces, we have that $W^{\prime}$ is also irreducible [BCR98, Theorem 2.8.3 (iii)]. In other words, $W=W^{\prime} \cap W^{\prime \prime}$ is a proper sub-variety of the irreducible variety $W^{\prime}$. Hence, $\operatorname{dim}(W)=\operatorname{dim}\left(W^{\prime} \cap W^{\prime \prime}\right)<\operatorname{dim}\left(W^{\prime}\right)$, equivalently, $\operatorname{codim}(W)>\operatorname{codim}\left(W^{\prime}\right)=2 n$. Hence, 
by Corollary 45 we have that, generically, the pre-image of $W$ under $j_{(2)}^{2} \widetilde{P}$ is empty. Since, by Proposition 56 Assumption $\mathcal{A}_{1}$ (which is necessary to guarantee that $\mathfrak{L}_{\mathrm{c}}^{\prime}$ is well-defined) is also generic, we imply that, generically, there is no distinct pair $q, q^{\prime} \in \mathfrak{C}$ such that $\pi_{\mathfrak{C}}(q)=\pi_{\mathfrak{C}}\left(q^{\prime}\right)$ and $q \in \overline{\mathfrak{L}_{\mathrm{c}}}$, equivalently, $\mathfrak{L}_{\mathrm{c}}^{\prime} \cap \mathfrak{L}_{\mathrm{n}}^{\prime}=\emptyset$ which proves the proposition.

We thus proved the following proposition that the silhouette of a generic surface in $\mathbb{R}^{n}$ satisfies all assumptions except for Assumptions $\mathcal{A}_{3(c)}$ and $\mathcal{A}_{5^{-}}$. However, based on previous results with three variables [IMP16b], we formulate the following conjecture.

Proposition 61. For a generic function $\widetilde{P} \in C^{\infty}\left(\mathbb{R}^{n}, \mathbb{R}^{n-2}\right)$, Assumptions $\mathcal{A}_{1}, \mathcal{A}_{2}, \mathcal{A}_{3(a)}, \mathcal{A}_{3(b)}, \mathcal{A}_{4}$, hold.

Conjecture 62. For a generic function $\widetilde{P} \in C^{\infty}\left(\mathbb{R}^{n}, \mathbb{R}^{n-2}\right)$, Assumptions $\mathcal{A}_{3(c)}$ and $\mathcal{A}_{5}^{-}$hold.

\section{Conclusion}

We proposed a regular square system that encodes the singularities of the plane projection of a curve in $\mathbb{R}^{n}$ under some assumptions, which we proved to be generic via transversality theory. For the case of plane projections of silhouette curves, we proved the genericity of only some of the assumptions and we conjecture the genericity of the others (Proposition 61 and Conjecture 62). We provided semi-algorithms that check whether a given curve satisfies our assumptions and, if they terminate, output isolating boxes of the singularities or the plane projection (possibly with spurious boxes under some weak assumptions). The drawback of our approach is that the number of variables is doubled, which is an issue for subdivision methods that are exponential in the dimension. We partially overcame this issue by applying subdivision schemes in the space of doubled dimension only locally in the neighborhood of the points that project onto singularities (Section 6.1).

A natural open question is the complexity of our semi-algorithms. It is worth noticing that our semi-algorithms use a subdivision approach with diameter distance tests as defined by Burr at al. [BGT20]. As such, it should be possible to study our complexities using the method of continuous amortization. This should yield explicit bounds for the case of polynomial input either in the worst case (as in [BGT20]), or in a smoothed complexity setting (as in [CETC19]).

\section{References}

[AGZV12] Vladimir Igorevich Arnold, Sabir Medgidovich Gusein-Zade, and Alexander Nikolaevich Varchenko, Singularities of differentiable maps. Volume 1, Modern Birkhäuser Classics, Birkhäuser/Springer, New York, 2012.

[Bôc64] Maxime Bôcher, Introduction to higher algebra, Dover Publications, Inc., New York, 1964.

[BCGY12] M. Burr, S. W. Choi, B. Galehouse, and C. K. Yap, Complete subdivision algorithms ii: Isotopic meshing of singular algebraic curves, Journal of Symbolic Computation 47 (2012), no. 2, 131 -152.

[BCR98] Jacek Bochnak, Michel Coste, and Marie-Francoise Roy, Real algebraic geometry, Springer, 1998. 


\begin{tabular}{|c|c|}
\hline Symbol & Description (see Section 2 unless specified otherwise) \\
\hline$C^{\infty}\left(\mathbb{R}^{n}, \mathbb{R}^{k}\right)$ & $\begin{array}{l}\text { Class of functions from } \mathbb{R}^{n} \text { to } \mathbb{R}^{k} \text { that are differentiable } \\
\text { infinitely many times }\end{array}$ \\
\hline$P=\left(P_{1}, \ldots, P_{n-1}\right)$ & A function in $C^{\infty}\left(\mathbb{R}^{n}, \mathbb{R}^{n-1}\right)$ \\
\hline $\mathfrak{C}$ & Curve defined by $P=0$ in an open box $B$ in $\mathbb{R}^{n}$ \\
\hline$\pi_{\mathfrak{C}}$ & Projection of $\mathfrak{C}$ onto the $\left(x_{1}, x_{2}\right)$-plane \\
\hline $\mathfrak{L}_{\mathrm{c}}$ & Set of points in $\mathfrak{C}$ where the tangent line is orthogonal to the $\left(x_{1}, x_{2}\right)$-plane \\
\hline $\mathfrak{L}_{\mathrm{n}}$ & $\begin{array}{c}\text { Set of points } q \text { in } \mathfrak{C} \text { such that the cardinality of the pre-image of } \pi_{\mathfrak{C}}(q) \text { is } \\
\text { at least two without counting multiplicities }\end{array}$ \\
\hline$\widehat{\mathfrak{L}}_{\mathrm{n}}$ & Set of $\left(q_{1}, q_{2}\right) \in \mathfrak{L}_{\mathrm{n}}^{2}$ such that $q_{1} \neq q_{2}$ and $\pi_{\mathfrak{C}}\left(q_{1}\right)=\pi_{\mathfrak{C}}\left(q_{2}\right)$ (Def. 12 ) \\
\hline$\widehat{\mathfrak{L}}_{\mathrm{c}}$ & Set of $\left(q_{1}, q_{1}\right)$ with $q_{1} \in \mathfrak{L}_{\mathrm{c}}$ (Def. 12 \\
\hline$\widehat{\mathfrak{L}}$ & $\widehat{\mathfrak{L}}_{\mathrm{n}} \cup \widehat{\mathfrak{L}}_{\mathrm{c}}$ (Def. 12 \\
\hline$T_{q} \mathfrak{C}$ & Line tangent to $\mathfrak{C}$ at $q$ \\
\hline$\overline{\mathfrak{C}}$ & Topological closure of $\mathfrak{C}$ \\
\hline$\overline{\pi_{\overline{\mathfrak{C}}}}, \overline{\mathfrak{L}_{\mathrm{c}}}, \overline{\mathfrak{L}_{\mathrm{n}}}, T_{q} \overline{\mathfrak{C}^{2}}$ & Analogous as $\pi_{\mathfrak{C}}, \mathfrak{L}_{\mathrm{c}}, \mathfrak{L}_{\mathrm{n}}, T_{q} \mathfrak{C}$ with respect to $\overline{\mathfrak{C}}$ intead of $\mathfrak{C}$ \\
\hline$S \cdot f\left(x_{1}, x_{2}, y, r, t\right)$ & Operator on $f: \frac{1}{2}\left[f\left(x_{1}, x_{2}, y+r \sqrt{t}\right)+f\left(x_{1}, x_{2}, y-r \sqrt{t}\right)\right]$ for $t \geqslant 0$ (Def. 95 \\
\hline$D \cdot f\left(x_{1}, x_{2}, y, r, t\right)$ & $\begin{array}{c}\text { Operator on } f: \frac{1}{2 \sqrt{t}}\left[f\left(x_{1}, x_{2}, y+r \sqrt{t}\right)-f\left(x_{1}, x_{2}, y-r \sqrt{t}\right)\right] \text { for } t>0 \text {, } \\
\left.\text { and } \nabla f\left(x_{1}, x_{2}, y\right) \cdot(0,0, r) \text {, otherwise (Def. } 9\right)\end{array}$ \\
\hline $\operatorname{Ball}(P)$ & Ball system $($ Thm. 11 \\
\hline$\Omega_{P}$ & $\begin{array}{l}\text { Maps a solution }\left(x_{1}, x_{2}, y, r, t\right) \text { of } \operatorname{Ball}(P) \text { to } \\
\text { the pair of points }\left(x_{1}, x_{2}, y \pm r \sqrt{t}\right) \text { in } \widehat{\mathfrak{L}} \text { (Def. } 14 \& \text { Fig. } 3 \text { ) }\end{array}$ \\
\hline$j^{r} f, J^{r}\left(\mathbb{R}^{n}, \mathbb{R}^{k}\right)$ & Jet of order $r$ of $f$ and space of jets of order $r$ of functions from $\mathbb{R}^{n}$ to $\mathbb{R}^{k}$ (Def. 42 \\
\hline$j_{(k)}^{r} f, J_{(k)}^{r}\left(\mathbb{R}^{n}, \mathbb{R}^{k}\right)$ & Multijet and space of multijets (Def. 46 ) \\
\hline
\end{tabular}

Table 3: Table of the main symbols used throughout this paper. 
[BGT20] Michael Burr, Shuhong Gao, and Elias Tsigaridas, The complexity of subdivision for diameter-distance tests, Journal of Symbolic Computation 101 (2020), $1-27$.

[BL13] Carlos Beltrán and Anton Leykin, Robust certified numerical homotopy tracking, Foundations of Computational Mathematics 13 (2013), no. 2, 253-295.

$\left[\mathrm{BLM}^{+}\right.$16] Yacine Bouzidi, Sylvain Lazard, Guillaume Moroz, Marc Pouget, Fabrice Rouillier, and Michael Sagraloff, Solving bivariate systems using rational univariate representations, Journal of Complexity 37 (2016), 34 -75.

[BPR06] Saugata Basu, Pollack Pollack, and Marie-Françoise Roy, Algorithms in real algebraic geometry, 2nd ed., Algorithms and Computation in Mathematics, vol. 10, Springer-Verlag, 2006.

[BV88] Winfried Bruns and Udo Vetter, Determinantal rings, Lecture Notes in Mathematics, vol. 1327, Springer-Verlag, Berlin, 1988.

[CETC19] Felipe Cucker, Alperen A. Ergür, and Josue Tonelli-Cueto, Plantinga-vegter algorithm takes average polynomial time, Proceedings of the 2019 on international symposium on symbolic and algebraic computation, 2019, pp. 114-121.

[CLO05] David A. Cox, John Little, and Donal O'Shea, Using algebraic geometry, Second, Graduate Texts in Mathematics, vol. 185, Springer, New York, 2005.

[CLO92] __ Ideals, varieties, and algorithms: An introduction to computational algebraic geometry and commutative algebra, Undergraduate texts in mathematics, Springer-Verlag New York-Berlin-Paris, 1992.

[Dem00] Michel Demazure, Bifurcations and catastrophes, Universitext, Springer-Verlag, Berlin, 2000. Geometry of solutions to nonlinear problems, Translated from the 1989 French original by David Chillingworth.

[DL14] Nicolas Delanoue and Sébastien Lagrange, A numerical approach to compute the topology of the apparent contour of a smooth mapping from $\mathbb{R}^{2}$ to $\mathbb{R}^{2}$, Journal of Computational and Applied Mathematics 271 (2014), 267 -284.

[DLZ11] Barry Dayton, Tien-Yien Li, and Zhonggang Zeng, Multiple zeros of nonlinear systems, Math. Comp. 80 (2011), no. 276, 21432168.

[GG73] Marty Golubitsky and Victor Guillemin, Stable mappings and their singularities, Springer-Verlag, New York-Heidelberg, 1973. Graduate Texts in Mathematics, Vol. 14.

[GVK96] Laureano González-Vega and M'hammed El Kahoui, An improved upper complexity bound for the topology computation of a real algebraic plane curve, Journal of Complexity 12 (1996), no. 4, $527-544$.

[Hon96] Hoon Hong, An efficient method for analyzing the topology of plane real algebraic curves, Mathematics and Computers in Simulation 42 (1996), no. 4, 571 -582. Sybolic Computation, New Trends and Developments.

[IMP16a] Rémi Imbach, Guillaume Moroz, and Marc Pouget, A certified numerical algorithm for the topology of resultant and discriminant curves, Journal of Symbolic Computation 80, Part 2 (2016), 285-306.

[IMP16b] Rémi Imbach, Guillaume Moroz, and Marc Pouget, Numeric and certified isolation of the singularities of the projection of a smooth space curve, Mathematical aspects of computer and information sciences, 2016, pp. 78-92.

[IMP18] Rémi Imbach, Guillaume Moroz, and Marc Pouget, Reliable location with respect to the projection of a smooth space curve, Reliab. Comput. 26 (2018), 13-55.

[Kea97] Ralph Baker Kearfott, Empirical evaluation of innovations in interval branch and bound algorithms for nonlinear systems, SIAM Journal on Scientific Computing 18 (1997), no. 2, 574-594.

[LSVY14] Jyh-Ming Lien, Vikram Sharma, Gert Vegter, and Chee Yap, Isotopic arrangement of simple curves: An exact numerical approach based on subdivision, Mathematical software - icms 2014, 2014, pp. 277-282.

[LSVY20] Jyh-Ming Lien, Vikram Sharma, Gert Vegter, and Chee Yap, Isotopic Arrangement of Simple Curves: an Exact Numerical Approach based on Subdivision, arXiv e-prints (September 2020), arXiv:2009.00811, available at 2009.00811 
[LY11] Long Lin and Chee Yap, Adaptive isotopic approximation of nonsingular curves: the parameterizability and nonlocal isotopy approach, Discrete \& Computational Geometry 45 (2011Jun), no. 4, 760-795.

[MKC09] Ramon E Moore, R Baker Kearfott, and Michael J Cloud, Introduction to interval analysis, SIAM, 2009.

[Neu91] Arnold Neumaier, Interval methods for systems of equations, Encyclopedia of Mathematics and its Applications, Cambridge University Press, 1991.

[Ra183] L. B. Rall, Mean value and taylor forms in interval analysis, SIAM Journal on Mathematical Analysis 14 (1983), no. 2, 223-238, available at https://doi.org/10.1137/0514019

[Rum10] Siegfried M. Rump, Verification methods: Rigorous results using floating-point arithmetic, Acta Numerica 19 (2010), $287-449$.

[Sny92a] John M Snyder, Generative modeling for computer graphics and cad: symbolic shape design using interval analysis, Academic Press Professional, Inc., 1992.

[Sny92b] John M. Snyder, Interval analysis for computer graphics, SIGGRAPH Comput. Graph. 26 (July 1992), no. 2, 121-130.

[Sta95] Volker Stahl, Interval methods for bounding the range of polynomials and solving systems of nonlinear equations, Ph.D. Thesis, 1995.

[vdHL18] Joris van der Hoeven and Robin Larrieu, Fast reduction of bivariate polynomials with respect to sufficiently regular gröbner bases, Proceedings of the 2018 acm international symposium on symbolic and algebraic computation, 2018, pp. 199-206.

[Whi43] Hassler Whitney, Differentiable even functions, Duke Mathematical Journal 10 (1943), 159-160.

[Whi65] _ L L L L _ pal properties of analytic varieties, Differential and Combinatorial Topology (A Symposium in Honor of Marston Morse), 1965, pp. 205-244.

[XY19] Juan Xu and Chee Yap, Effective subdivision algorithm for isolating zeros of real systems of equations, with complexity analysis, Proceedings of the 2019 international symposium on symbolic and algebraic computation, 2019, pp. 355-362. 